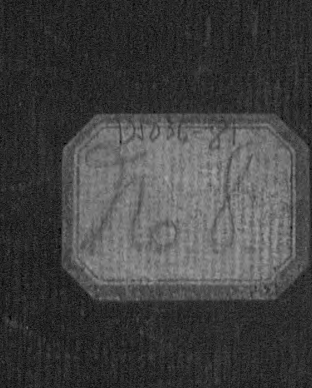
5.

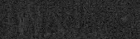


An $\frac{5036-5081}{12}$ 

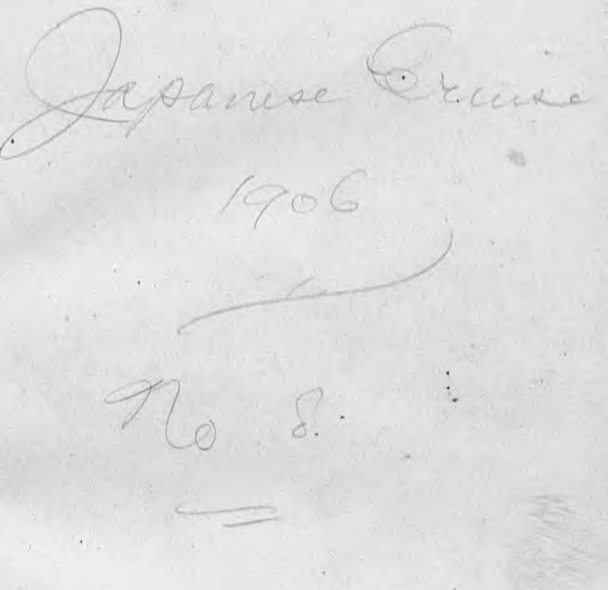
No. 7036 Date 2

Jac Machine. Reel.

Turns

Cor +

Depth

Shot or lead 3 ?

Bottom $\rightarrow$

Bottom temperature

No. of thermometer $/$ Cor. . . C

Corrected temperature 37

Air a Surface

Drift

Trawl or dredge

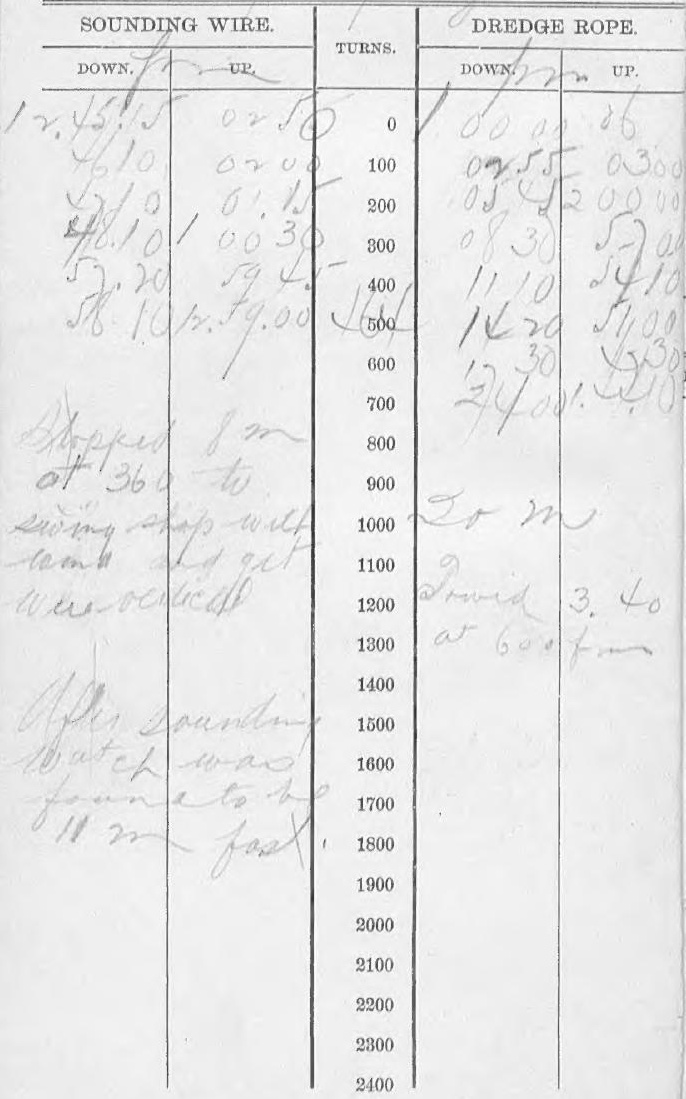


No. $\mathrm{H} \& \mathrm{~g}=3$ Date 2

\section{Machine. Reel.}

\section{Turns \\ Cor. + + \\ Depth}

Shot or lead .

Bottom

Bottom temperature 38

No. of thermometer / o , Cor..., I

Corrected temperature 37.9

Air Surface Drift

Trawl or dredge

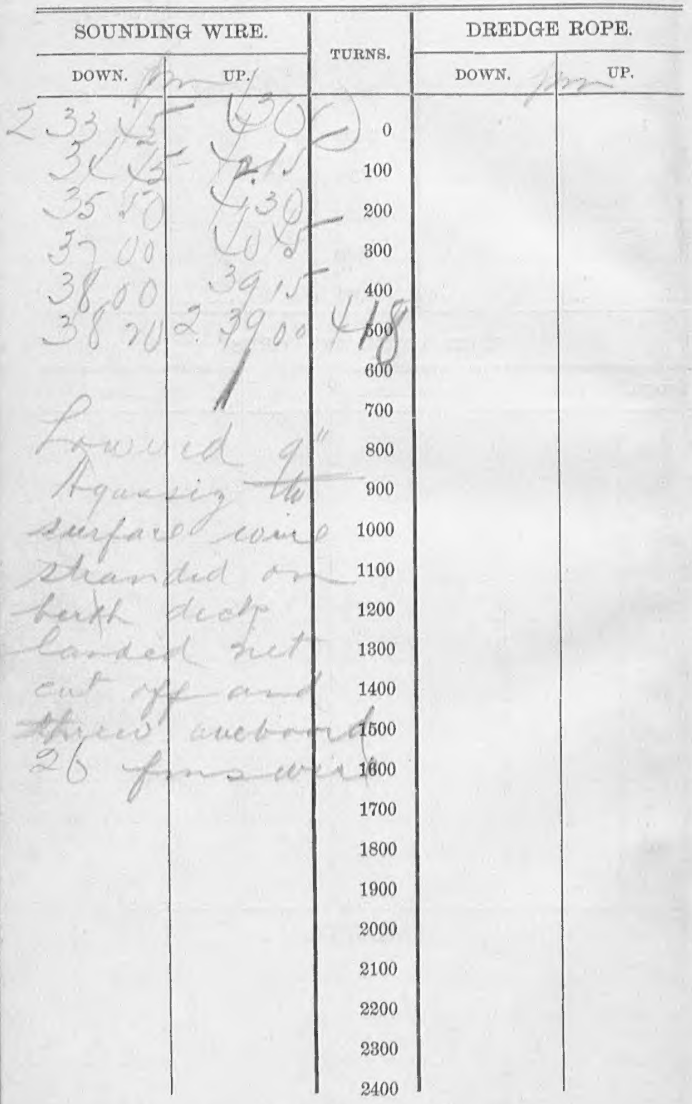




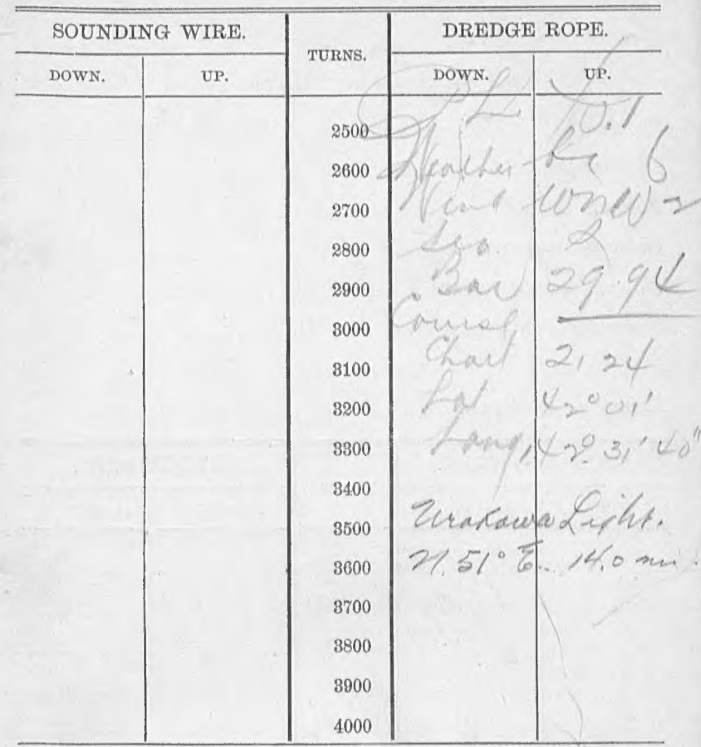

SERIAL TEMPERATURES.

\begin{tabular}{|c|c|c|c|c|}
\hline DEPTH. & TEMP. & NO. OF THER. & COR. & COR. TEMP. \\
\hline 25 & & & & \\
\hline 50 & & & & \\
\hline 100 & & & & \\
\hline 200 & & & & \\
\hline 300 & & & & \\
\hline 400 & & & & \\
\hline 500 & & & & \\
\hline 600 & & & , & \\
\hline 700 & & & & \\
\hline 800 & & & & '. \\
\hline 900 & & $n^{2}$ & & \\
\hline 1000 & & & & \\
\hline
\end{tabular}


Sue Machine. Reel.
Tums
Cor +
Deptha
Shot or lead $95^{-4}$ \& Cuy

Bottom

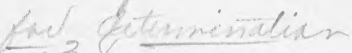

Bottom temperature

- No. of themometer.

Cor: /

Corrected temperature, 37,9

Air Surface Drift

Trawl or dredge o \& Rquats?

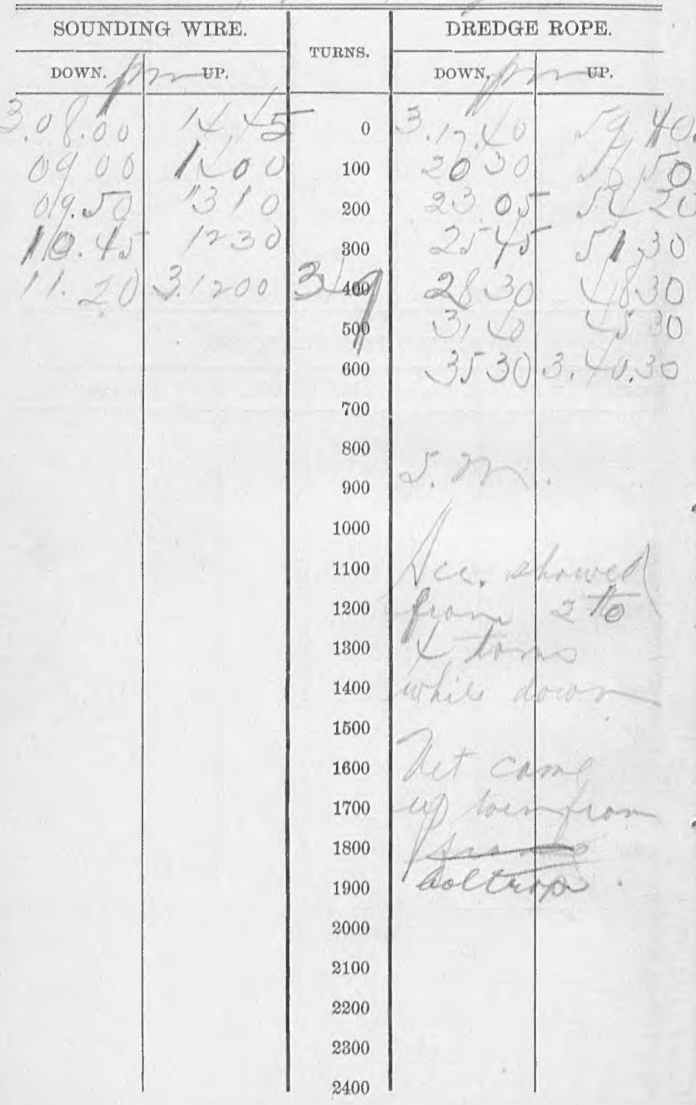




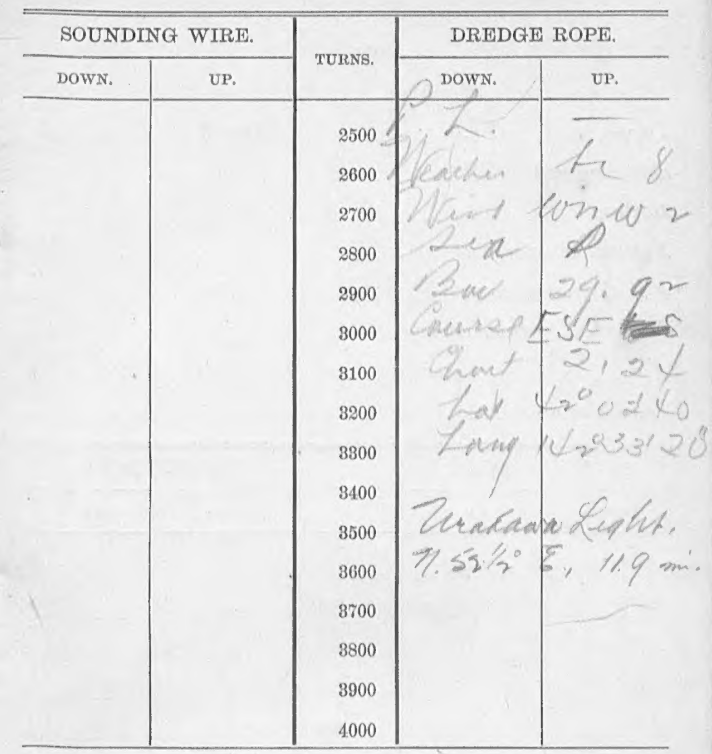

SERIAL TEMPERATURES.

\begin{tabular}{|c|c|c|c|c|}
\hline DEPTH. & TEMP. & NO. OF THER. & COR. & COR, TEMP. \\
\hline 25 & & & & \\
\hline 50 & & & & \\
\hline 100 & & & & \\
\hline 200 & & & & \\
\hline 800 & & & & \\
\hline 400 & & & & \\
\hline 500 & & & & \\
\hline 600 & & & & \\
\hline 700 & & & & \\
\hline 800 & & & & . \\
\hline 900 & & & & \\
\hline 1000 & & & & \\
\hline
\end{tabular}

REMARKS: 
No

- No. 10.4-0.38 Date Qct, 2,1906

Levear Machine. Reel. Lucar
Turns
Cor.+
Depth I y f fath
Shot on lead $33^{5}$

Bottom

Bottom temperature $37,2^{\circ}$

No. of thermometer /o $8 / \mathrm{s}^{-} \quad \mathrm{Cor} \rightarrow / 0$

Corrected temperature $3 \% /^{\circ}$

Air $5^{\circ} 3^{\circ} \quad$ Surface $64^{\circ}$ Drift

Trawt or dredge 9' But Q1 - B?.

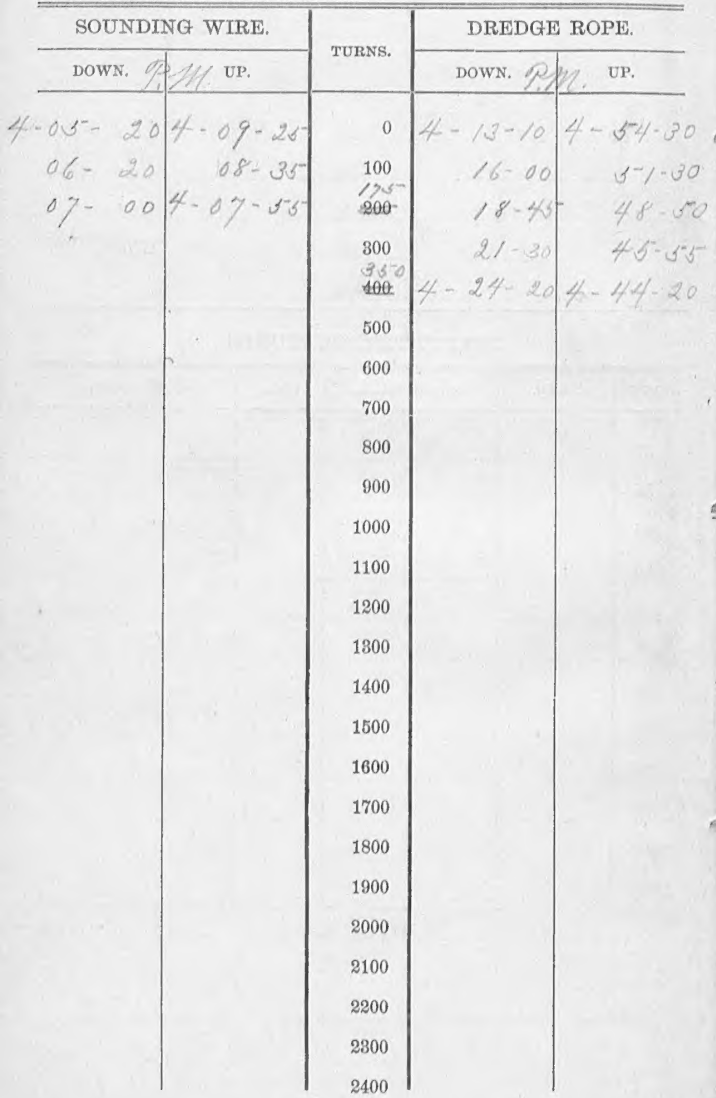




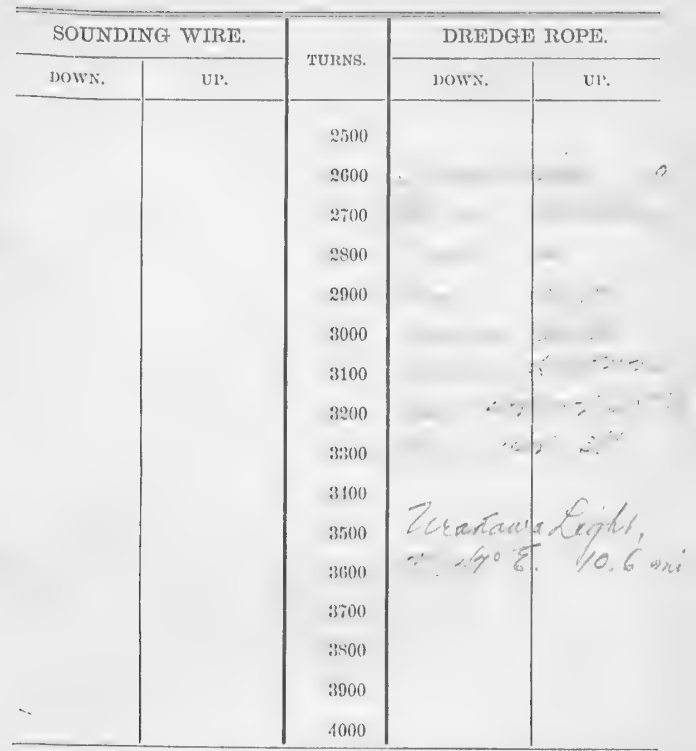

SERIAL TEMPERATURES.

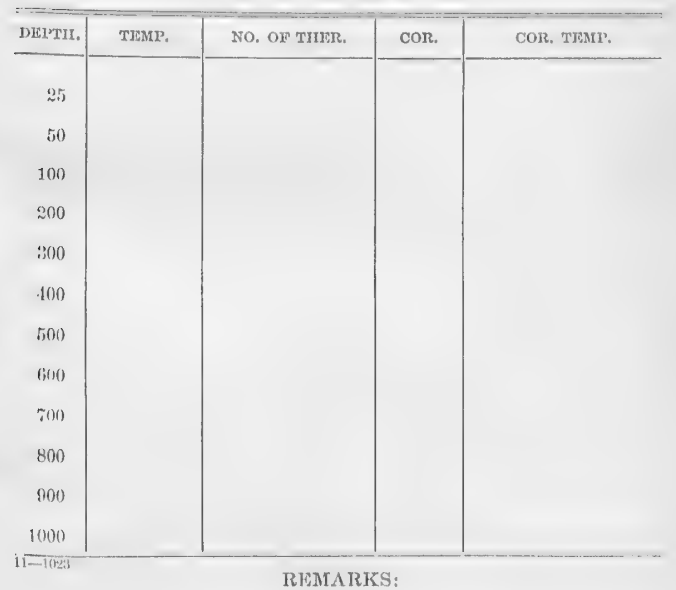




\begin{tabular}{|c|c|c|c|c|}
\hline \multicolumn{2}{|c|}{ SOUNDING WIRE. } & \multirow{2}{*}{ TUIRNS. } & \multicolumn{2}{|c|}{ DREDGE ROPE. } \\
\hline DOWN. & ur. & & DOWs. & ur. \\
\hline & & $\begin{array}{l}2500 \\
2000 \\
2 \% 00 \\
2800 \\
2900 \\
3000 \\
3100 \\
3200 \\
3300 \\
3100 \\
3500 \\
3600 \\
3700 \\
3500 \\
3900 \\
4000\end{array}$ & 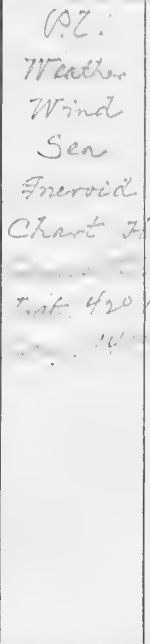 & $\begin{array}{c}73.7 \\
\text { on } 10 \\
\text { NE } 2 \\
5 . \\
29.66 \\
0.2026 \\
N N E \\
100\end{array}$ \\
\hline
\end{tabular}

SEIRIAL TEMPPRATUIRES.

\begin{tabular}{|c|c|c|c|c|}
\hline DEI'TIE. & 'THall. & No, of THIIR. & colt. & COH. TI:AH'. \\
\hline 25 & & & & \\
\hline 50 & & & & 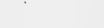 \\
\hline 100 & & & & \\
\hline 200 & & & & \\
\hline 300 & & & & \\
\hline 400 & & & & \\
\hline 500 & & & & \\
\hline (6)0 & & & & \\
\hline 700 & & & & \\
\hline 800 & & & & \\
\hline 900 & & & & \\
\hline 1000 & & & & \\
\hline
\end{tabular}


No. So vo Date 30 ot. 06.

'tuens Machine. Rech. Luene
Turns
Cor + +
Depth 269 fins.

Shot ax toat $35-x$ and S. S. Omp.

Bottom

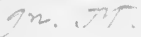

Bottom temperature

37.2

No. of thermometer

$108 / \sqrt{3}$

Cor. _- .

Corrected temperature

$37 \% 1$

Air 's]० Surface 620

Drift

Trawt or dredge G. $176 .-8 \mathrm{Z}$

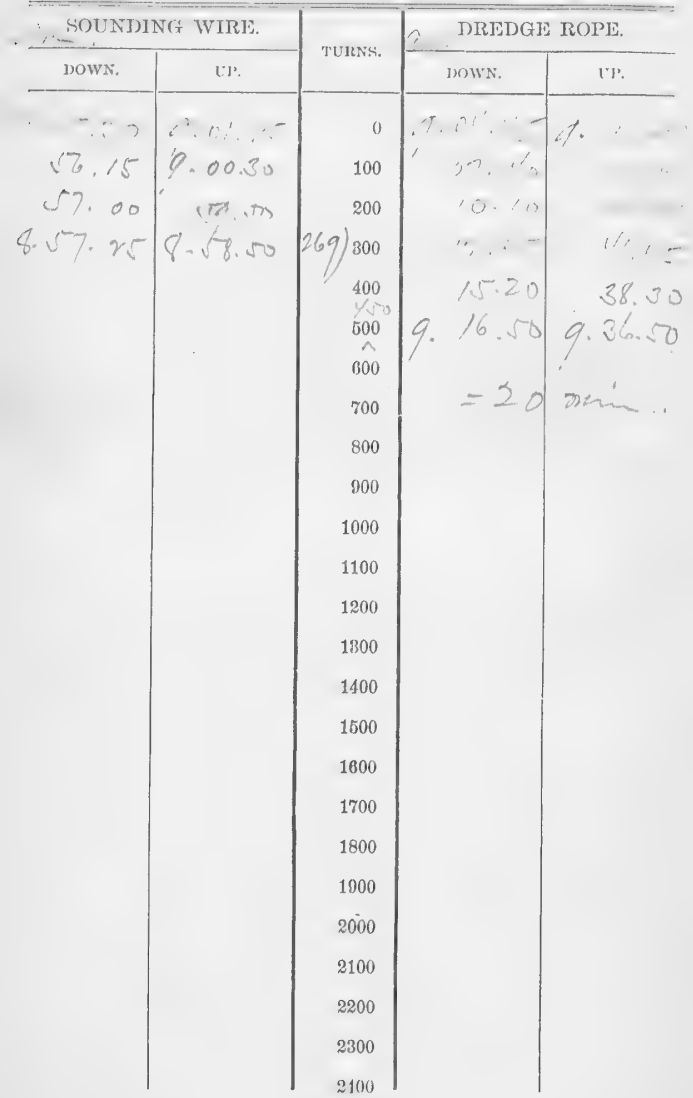




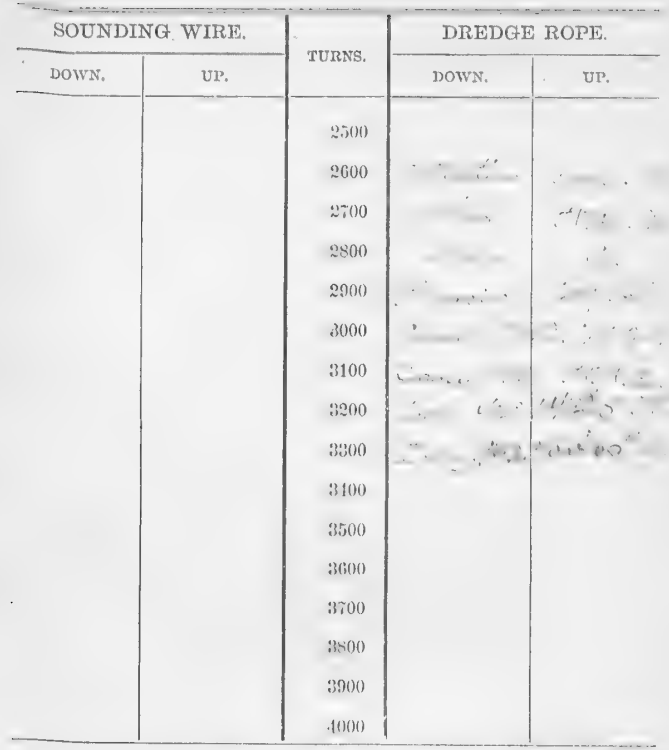

SERIAL TEMPERATURES

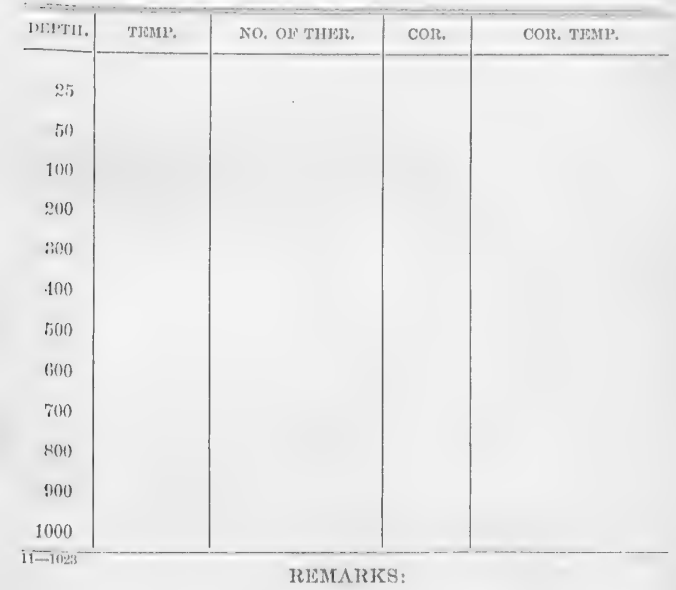


cot in

Wo. $0.50 .4 /$ Date 30 ct. 06.

- towezo Machine. Reel. Zuken

Iurns Corit Depth 140 fors,

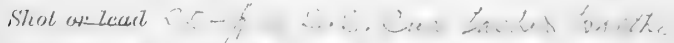

Jiottom,

Bottom temperature

$4: 2$

No, of thermometer / $O Q \mathrm{~V}^{-}$. Cor. _ . ।

Corrected temperature

41:1

Air $52^{\circ}$ Surface $62^{\circ}$ Drift

Trawt or aredge $q^{\prime} \mathrm{St6.232.}$

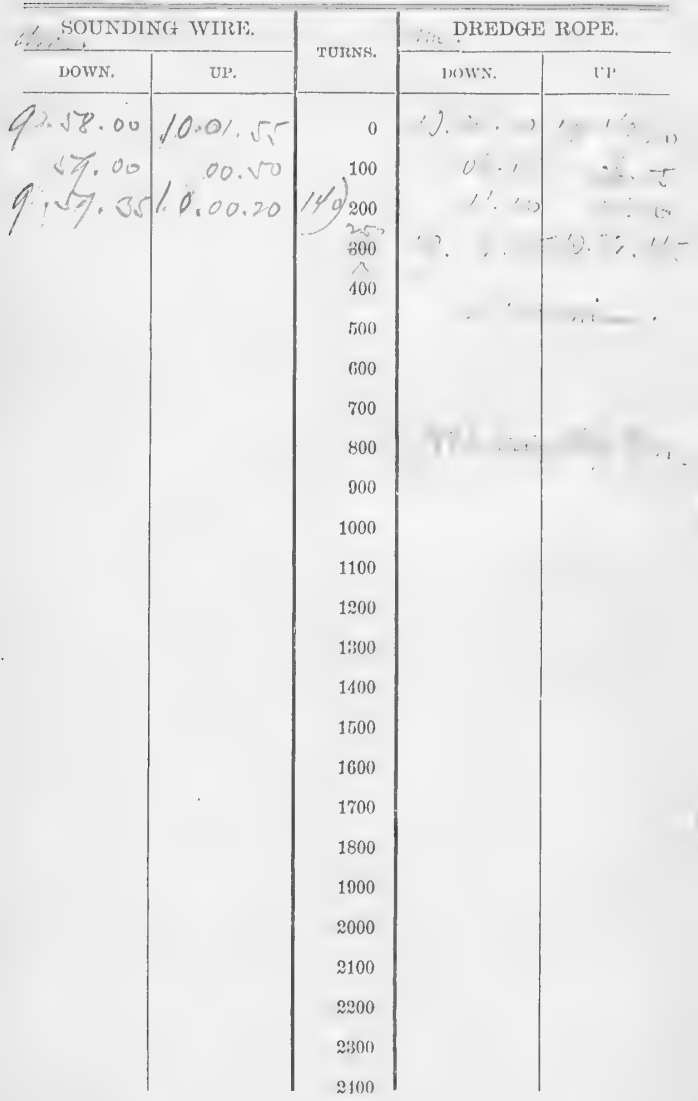


$507, j 1$

iñ. Sivis Date

3

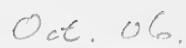

j)

Machine.

Reel.

Turns Cor.t

Shot om terul

$2 \mu$

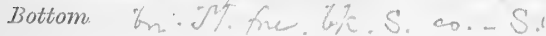

Bottom temperature.

No. of themmometer

Corrected temperature

Cor. - . 2

Air

Surface

$7 \%, 8$

Trawl or dredge on, =7assiz?

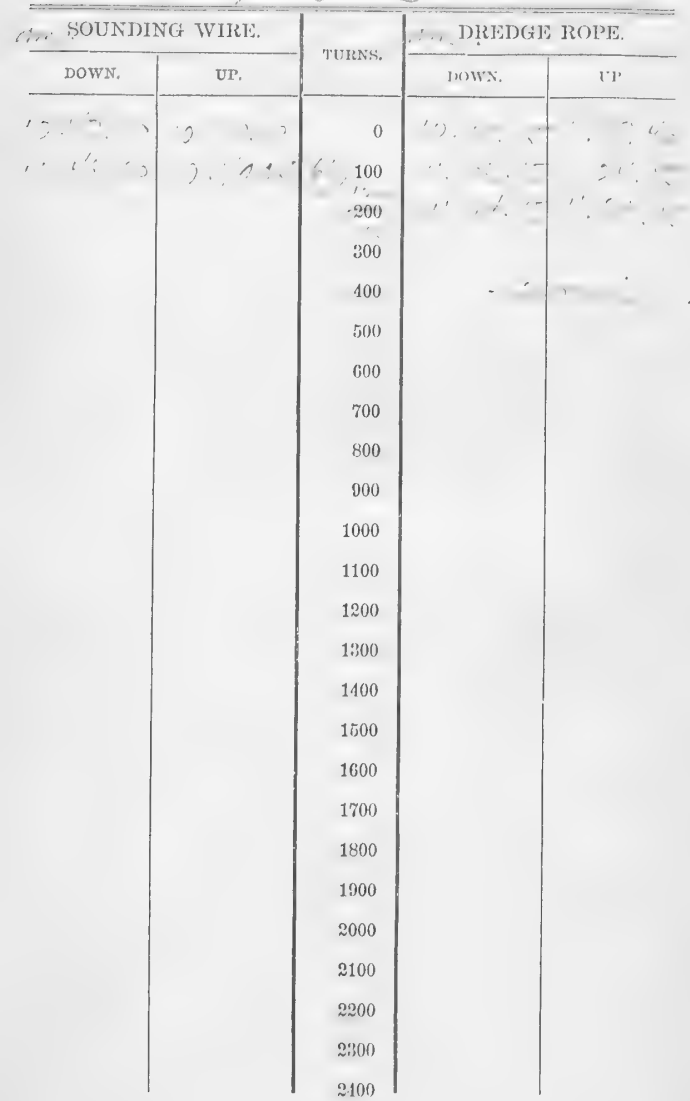




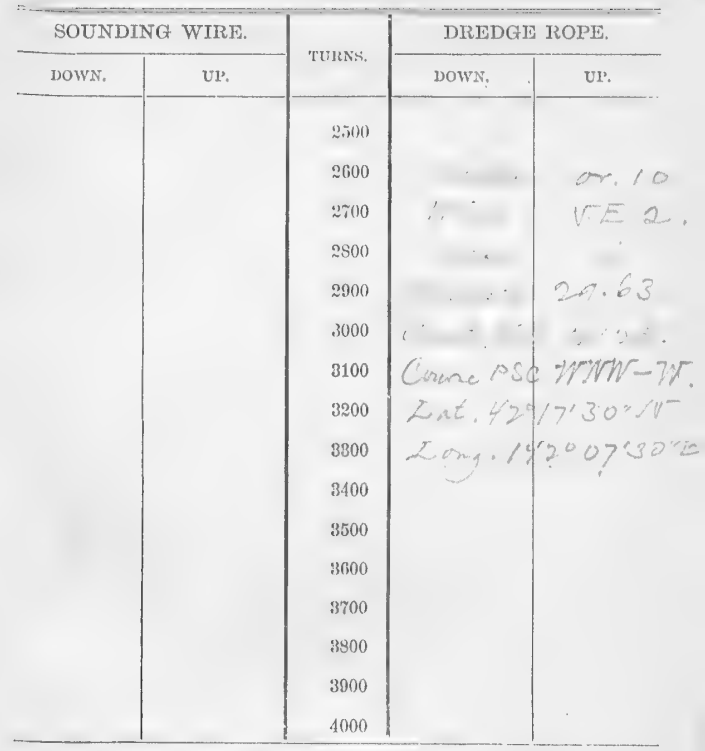

SERLA TEMIPRA'TURES

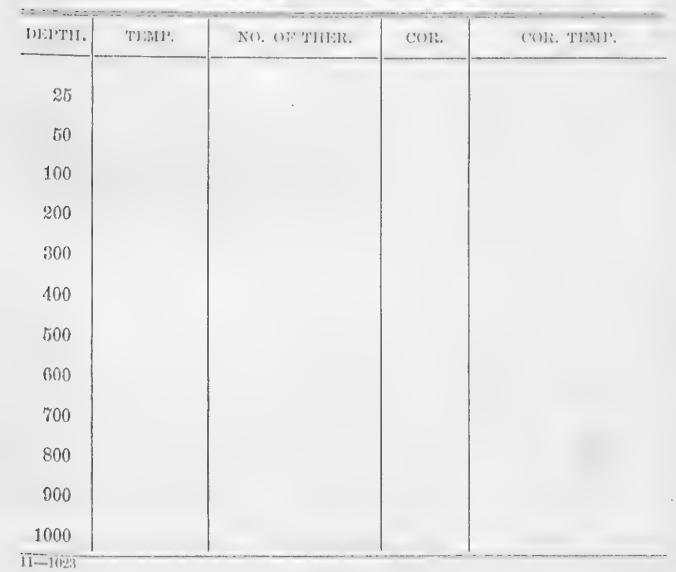

IREMARIES: 


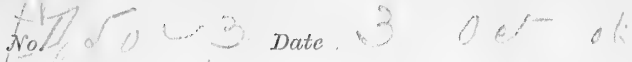
Hoct... Machine, Reel.
Turnes
Cor: +
Depth,
Shot or lead $3 \mathrm{~J}$
$5(\cdots)$

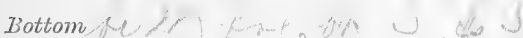

Bottom temperature

No. of thermometer / Cor.

Corrected temperature

Air.

Surface

6. Drift

Irawt or dredge

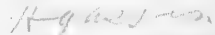

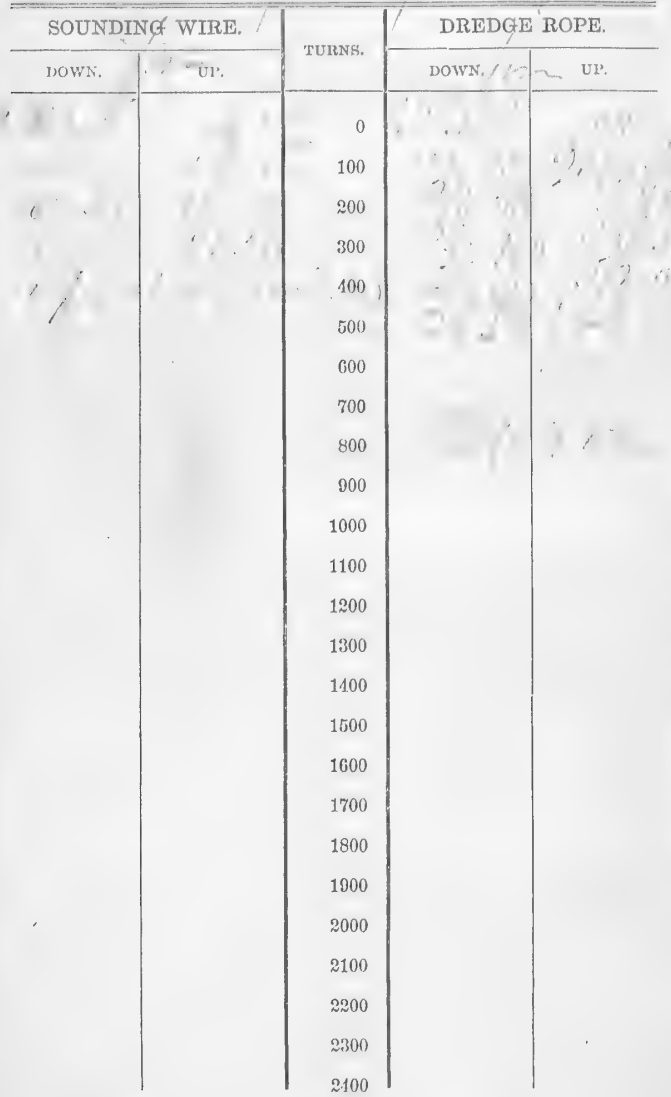


. No. i 3 Date "ofacen? Machine. Reet.

Turns Cor,t is Depth is o c/

Shot or lead

Bottom

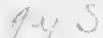

4) $($ in, $t)$

Bottom tempierature

No. of thermometer

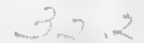

$+$

Corrected temperature

Air 2 Surface

Irawl or dredge

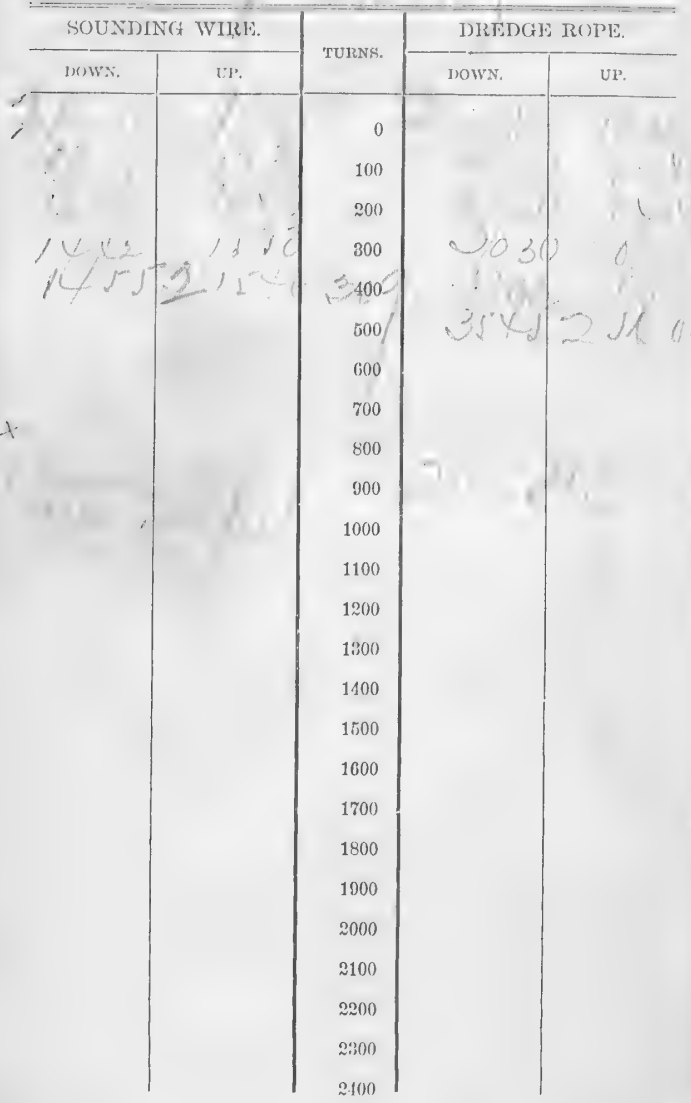




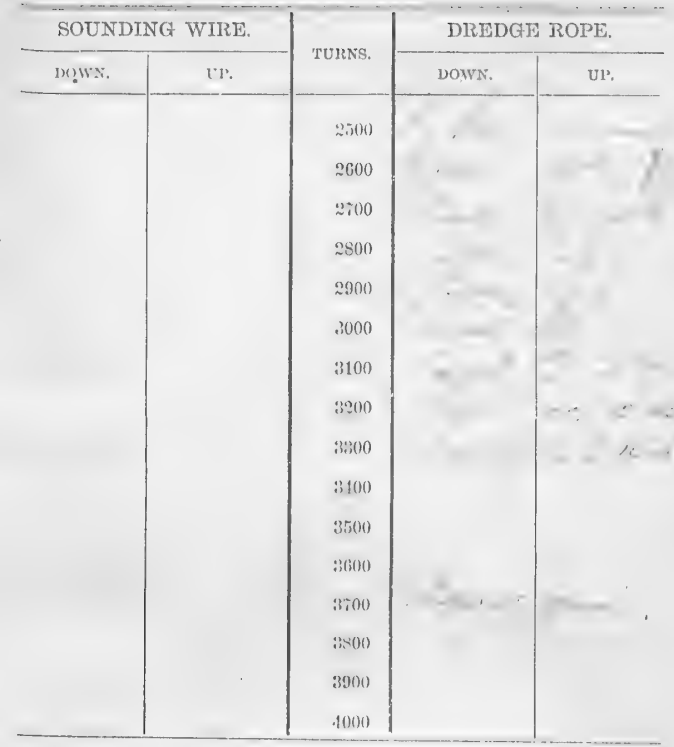

SLRIAL TEMIPERATURES

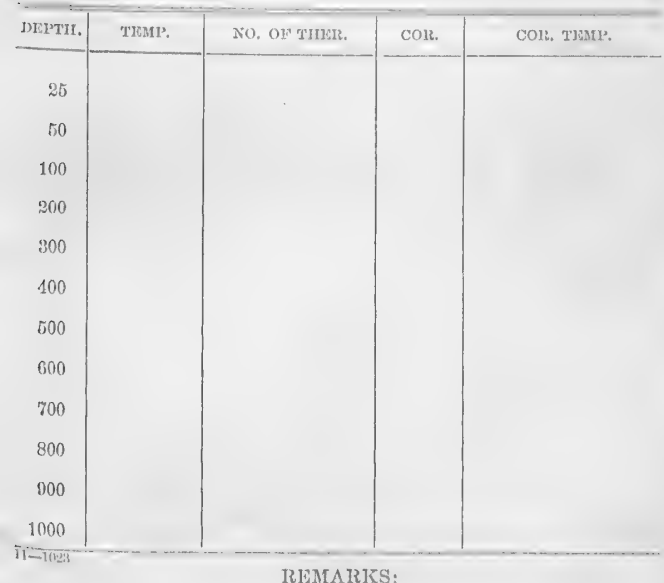

REMARIRS: 
Shot or lead 5 , is in

Bottom , the? ?

Bottom temperature

Jo. of the

No. of thermometer

Corrected temperature

Aiv of Surfacol

(?) IS $5 \mathrm{Cor}$. . I

trayot or tredge

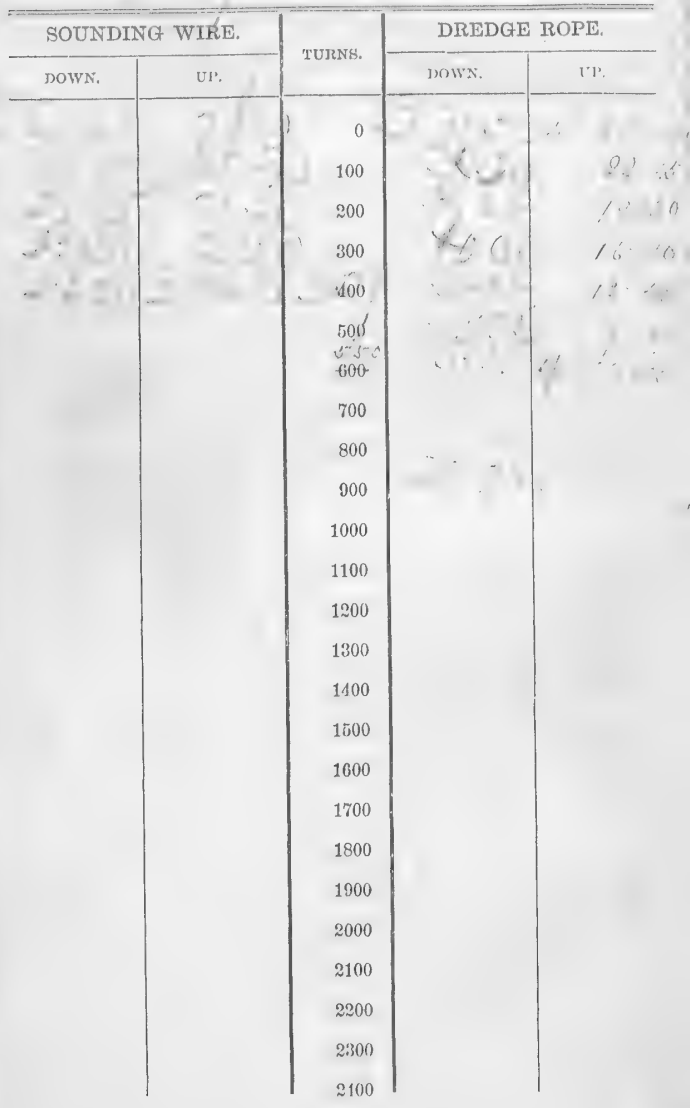




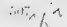

A

Date : 10 Octäer 1906

- Incaro Machine. Reel. Iura

Thrns Cor.t Depth 82 frio.

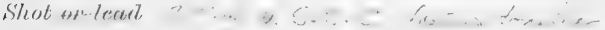

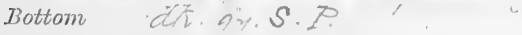

Bottom temperature

$5 \%$

No. of thermometer

C'or: _. .

Corrected temperature

50.8

Air vz" Surface $63^{\circ}$ Drift

Trawl or dredge $q^{\prime}=725$. $18 \mathrm{ZZ}$.

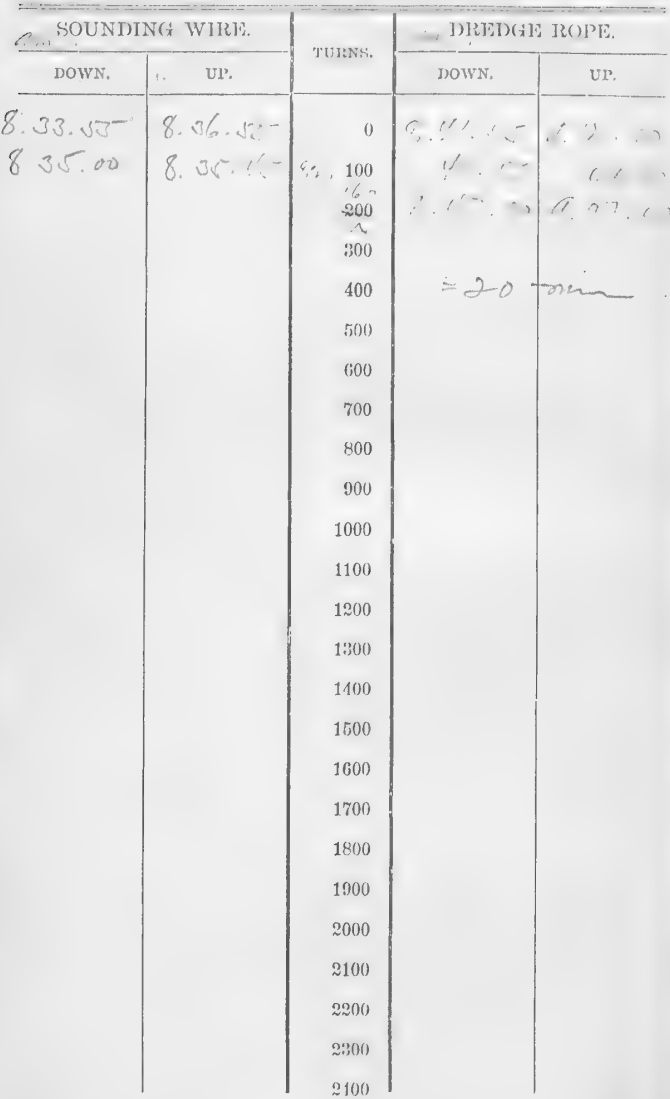




\begin{tabular}{|c|c|c|c|c|}
\hline \multicolumn{2}{|c|}{ SOUNDING WIRE. } & \multirow{2}{*}{ TU1KS: } & \multicolumn{2}{|c|}{ DREDGE ROPE. } \\
\hline DOWN. & vr. & & DOWN. & ve. \\
\hline & & 2500 & $\theta .2$ & 16.0 \\
\hline & & 2600 & .7 eather & thom 9 \\
\hline & & 2700 & $M$ ins & $\sqrt{E} E 1-2$ \\
\hline & & 2800 & Lex & $S$ \\
\hline & & 2900 & atrestid & 30.30 \\
\hline & & 3000 & $C_{12} a_{2}$ & \%.0. 2100 \\
\hline & & 3100 & Coxve PSC & -SSE - S \\
\hline & & 3200 & LAE. $38^{\circ}$ & $15^{\prime} 07^{\prime \prime} \sqrt{.}$ \\
\hline & & 3300 & & ? \\
\hline & & 3400 & Ruintis S & in $\alpha_{i j} \bar{e}$ \\
\hline & & 3500 & $\therefore \quad-1$ & $\because 1 \quad \therefore$ \\
\hline & & 3600 & $\% 25$ & 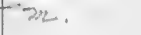 \\
\hline & & 8700 & & \\
\hline & & 3800 & & \\
\hline & & 3900 & & \\
\hline & & 1000 & & \\
\hline
\end{tabular}

SERIAI, TEMPERATURES

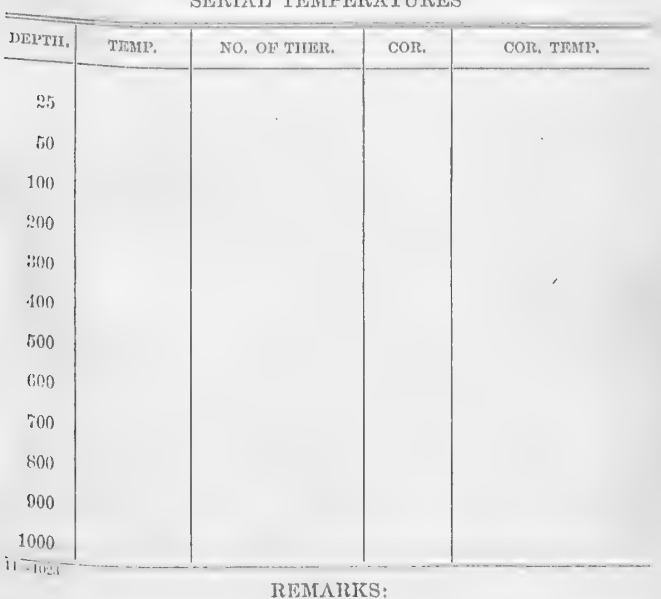

REMAIRKS: 
10 Oct. OG.

$\checkmark$ Eucmo Machine. Reel. Lueceo
Turns
Cor.+
Depth 107 frwo.

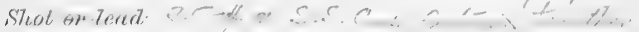

Bottom dx. Q S. P.

Bottom temperature

4.9.8

No of thermometer $108 / \sqrt{5}$, Cor. - . 2

Corrected temperature

Air $7^{\circ}$ Surface 63. Drift

Trawt or aredge \&' $916 .-13 t$.

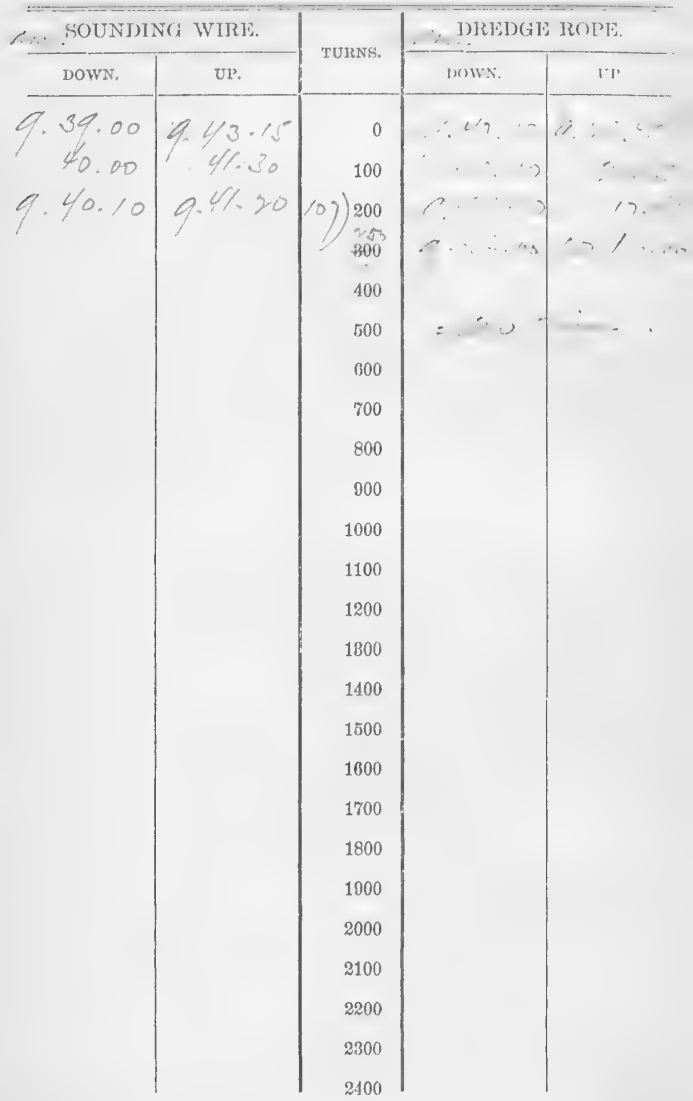




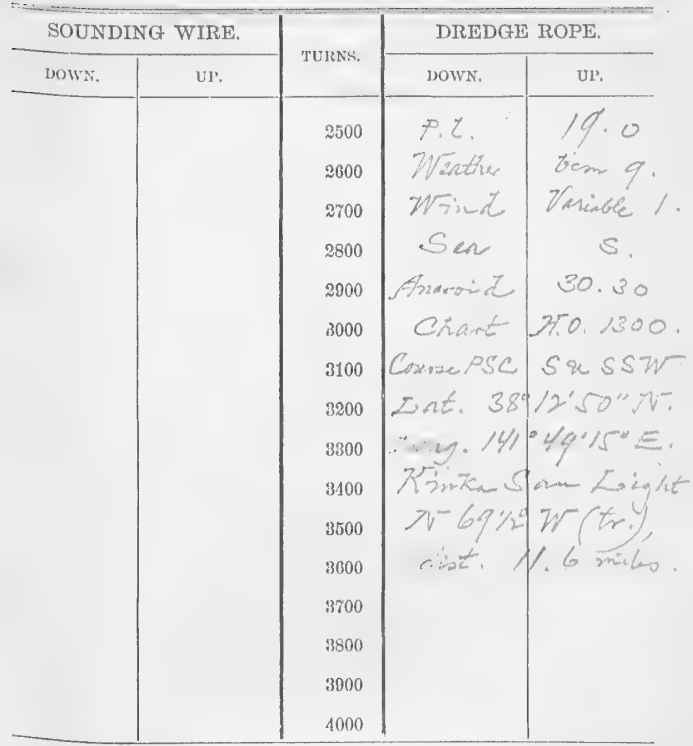

SERIAL TEMPERATURES

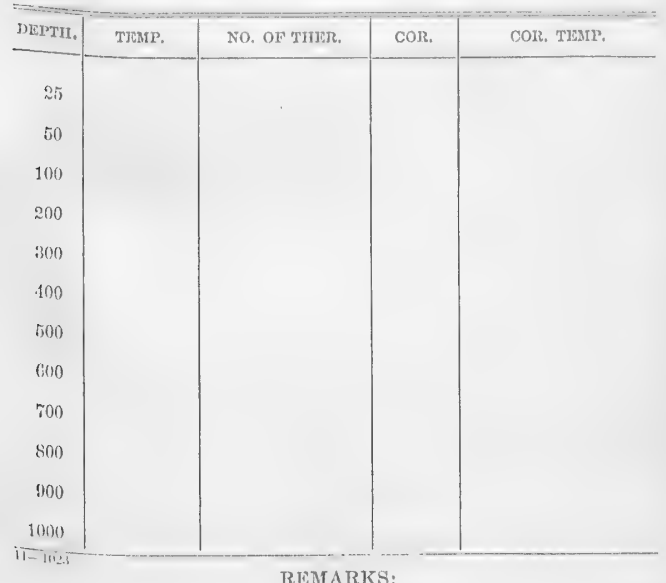


Nio. 0.5048 . Date 10 Oct. 06.

j) Inens Machine. Reel. Inues
I'urns
Cor. +
Depth 129 frow

Shot or tead 35 A And S.S.C. frokines tongether.
Bottom
dK. 1\%. S
S.2.

Bottom temperature " $40: 8$

No. of thermometer $108 / \sqrt{5}$. Cor. _ 1 I

Corrected temperature $40: 2$

Air $60^{\circ}$ Surface $63^{\circ}$ Mrifl!

Trawl or dredge $q$. Alt6:- $32 k$.

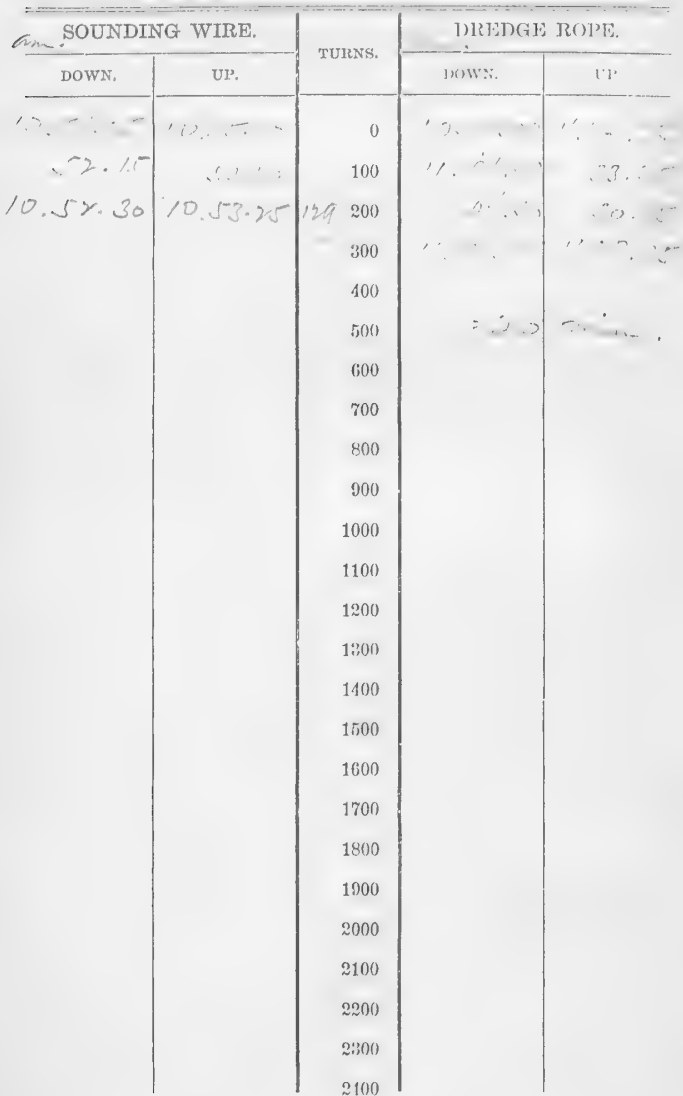




\begin{tabular}{|c|c|c|c|c|}
\hline \multicolumn{2}{|c|}{ SOUNDING WIRE. } & \multirow{2}{*}{ TURS. } & \multicolumn{2}{|c|}{ DREDGE ROPE. } \\
\hline DOWN. & Ur. & & Down, & Ur. \\
\hline & & $\begin{array}{l}2500 \\
2600 \\
2700 \\
2800 \\
2000 \\
3000 \\
3100 \\
: 300 \\
3300 \\
3400 \\
3500 \\
33600 \\
3300 \\
: 3500 \\
3900 \\
19000\end{array}$ & 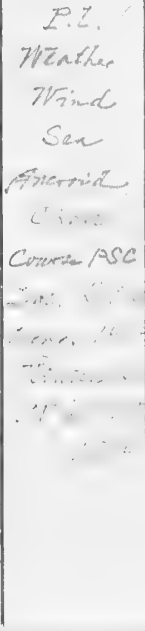 & 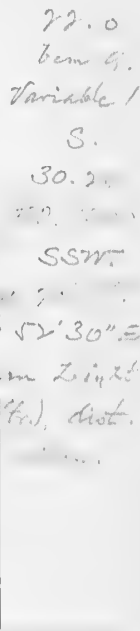 \\
\hline
\end{tabular}

SERTAL TEMIPERATURES

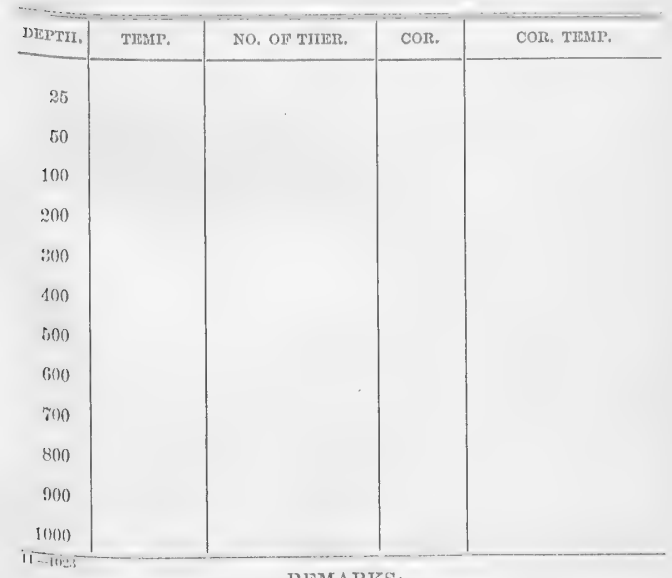

REMARKS: 
$\lambda^{\circ} \%$.

$$
\text { Teci. Machine Reel. }
$$
Turns
Cor", +
Depth

Shot or tead

Bottom

$=$

Bottom temperature

No. of thermometer

Cor. . . /

Corrected tomperature

Air t Surface is Drift

Trawl or aredge -

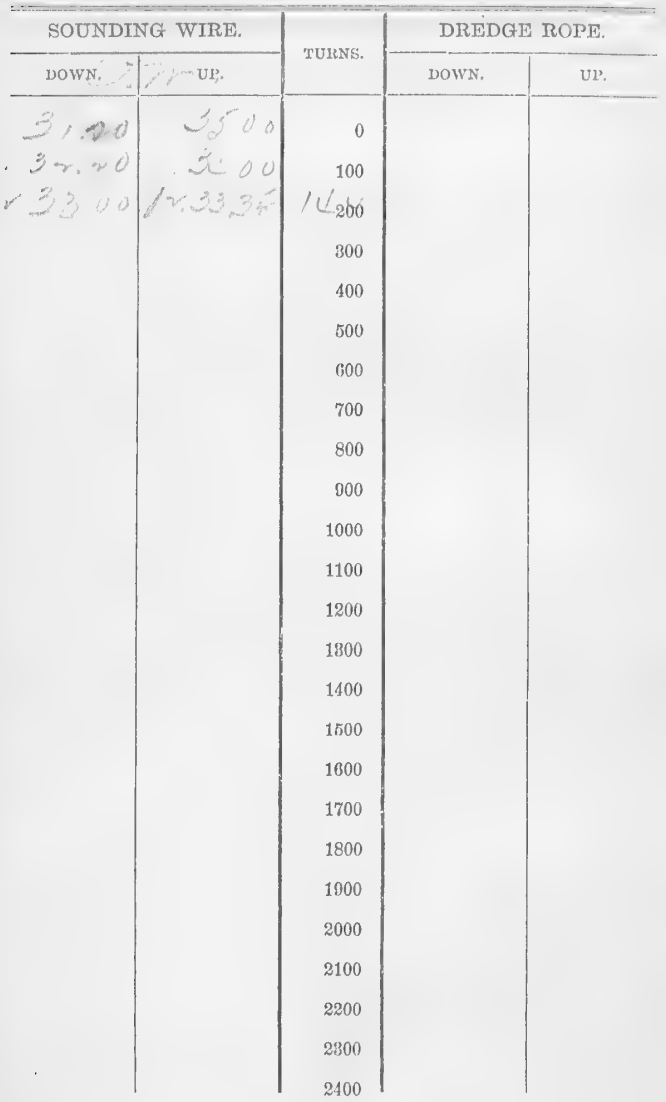




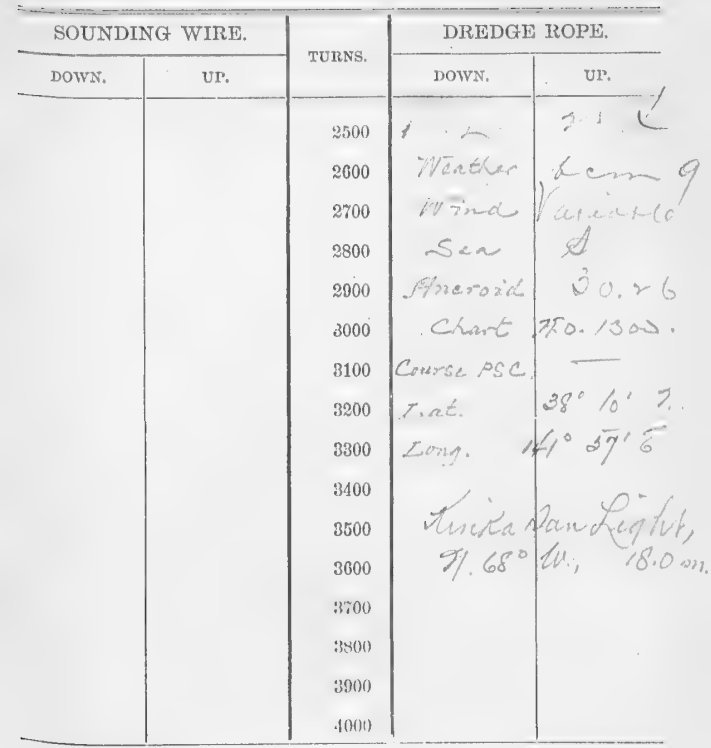

SERIAL TLMPERATURES

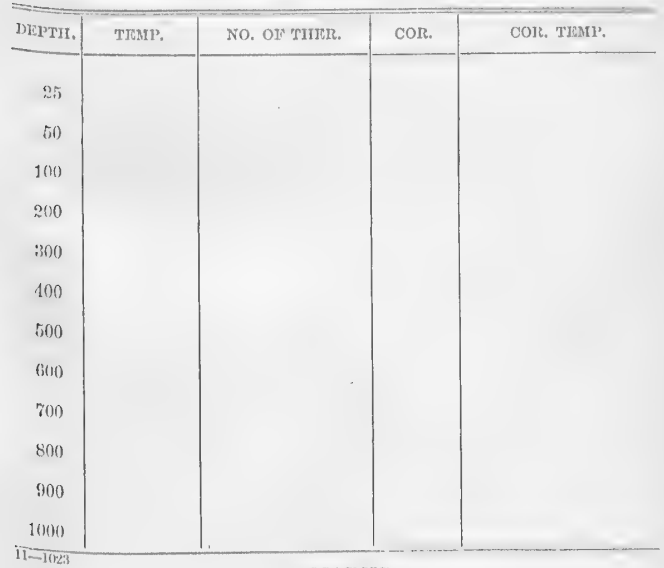

REMARKS: 


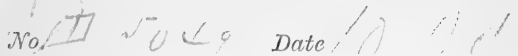

The Machine. Reel.

Turns Cor.t Depth / f

Shot or lead - o

Bottom ob S $S$ S

Bottom temperature

No. of thermometer

Corrected temperature

Air * Surfacé Drift

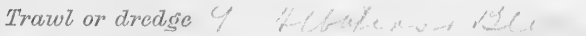

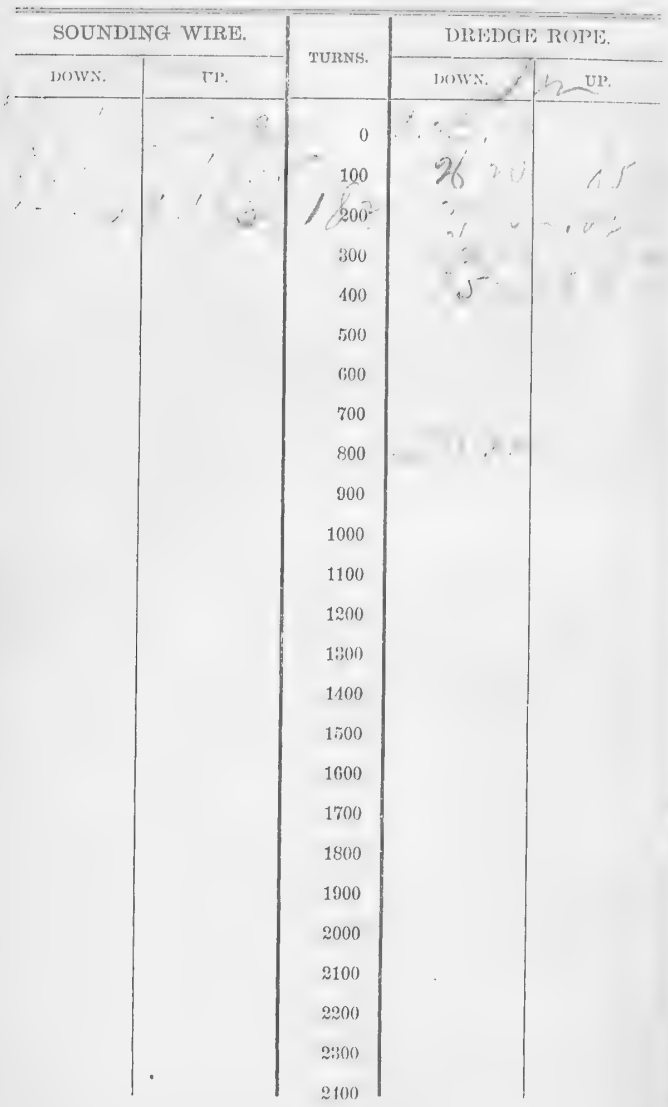




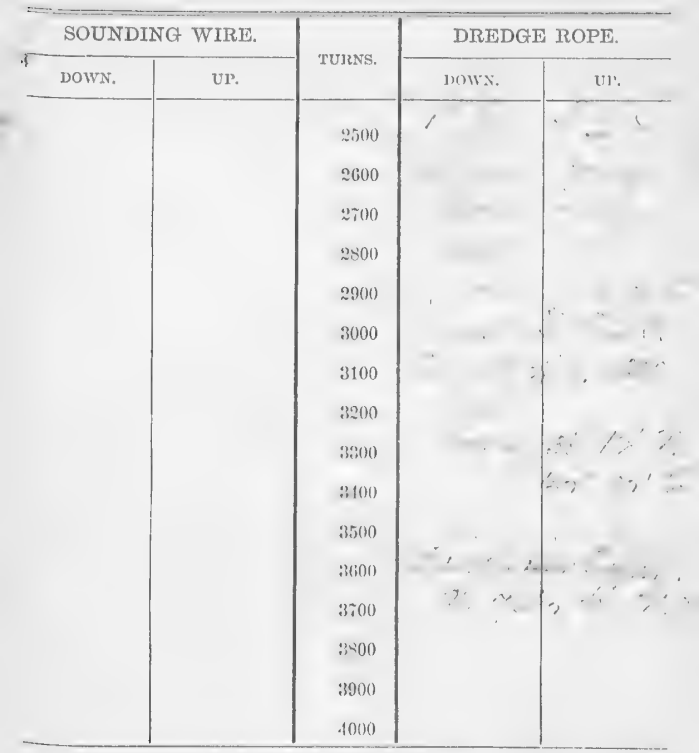

SERIAL TEMPERATURES.

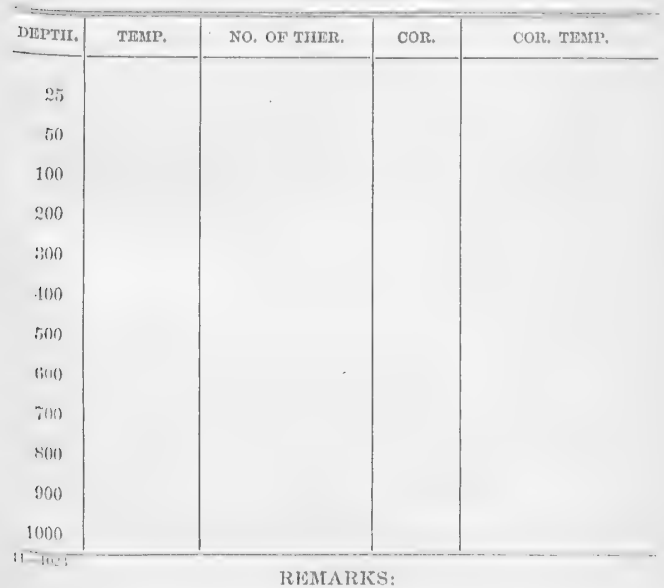


607,1211

So, D o T Date

Whe Machine, Rect.

Turns Cor.t a. Depth 26

Shot or lead - 3 che

Bottom $d /$ a $S$. S \& S S in

Bottom temperdture

No. of thermometer.

Cor. - 1

Corrected temperature

Air of Surface. $C^{\circ}$ Drift

Trawl or dredge

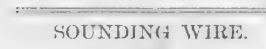

INOWx.
DIREDGE ROPE.

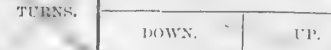

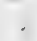

[I?.
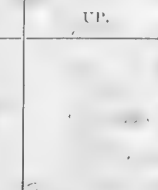

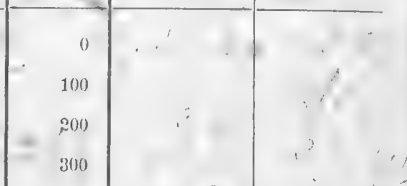

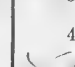

400)

$1 \div$

.

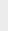




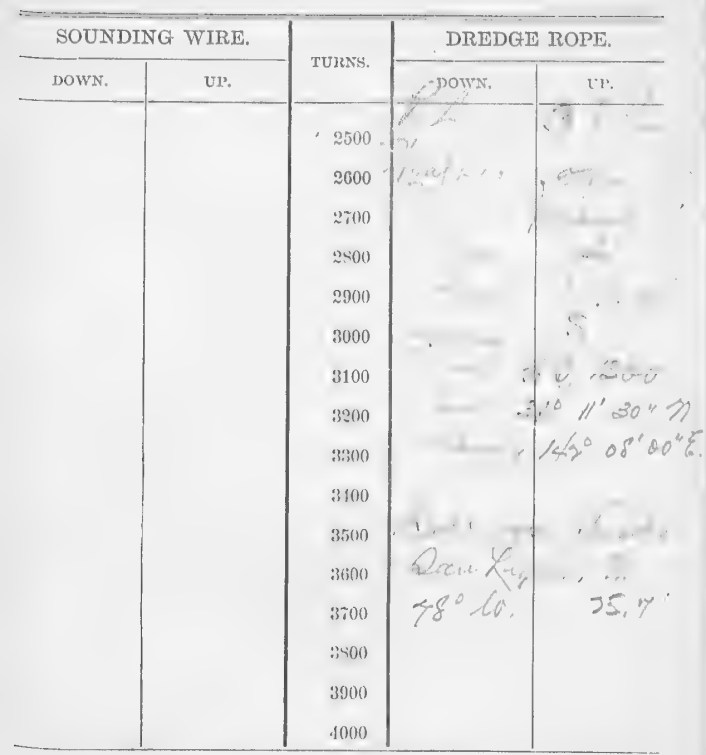

SERIAL TEMPERATURES.

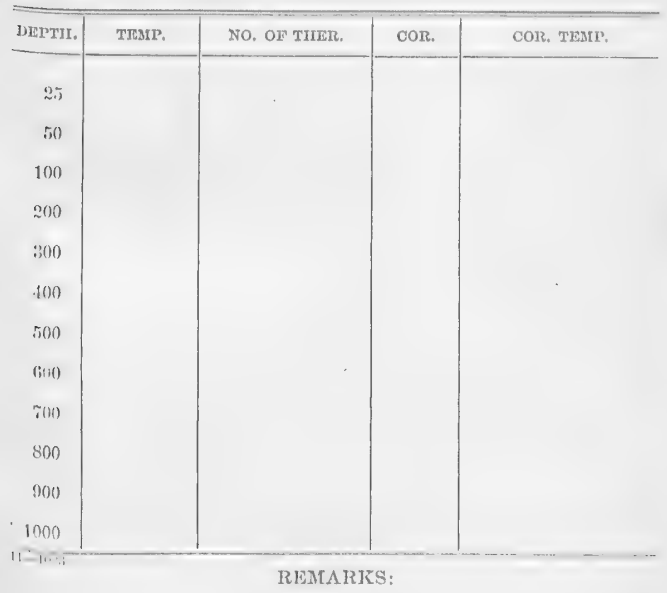


No. 10. 5051 Date Oet. 10,1906 Lucae Machine. Reel. Lucat Turns Cor.t Depth 399 f eth.

Shot om-tertech a:

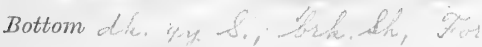

Bottom temperáture 38.

No. of thermometer $108 / \mathrm{s}^{-5} \quad$ Cor, - $)^{\circ}$

Corrected temperature $38.1^{\circ}$

Air $37^{\circ}$ Surface $63^{\circ}$ Drift

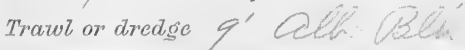

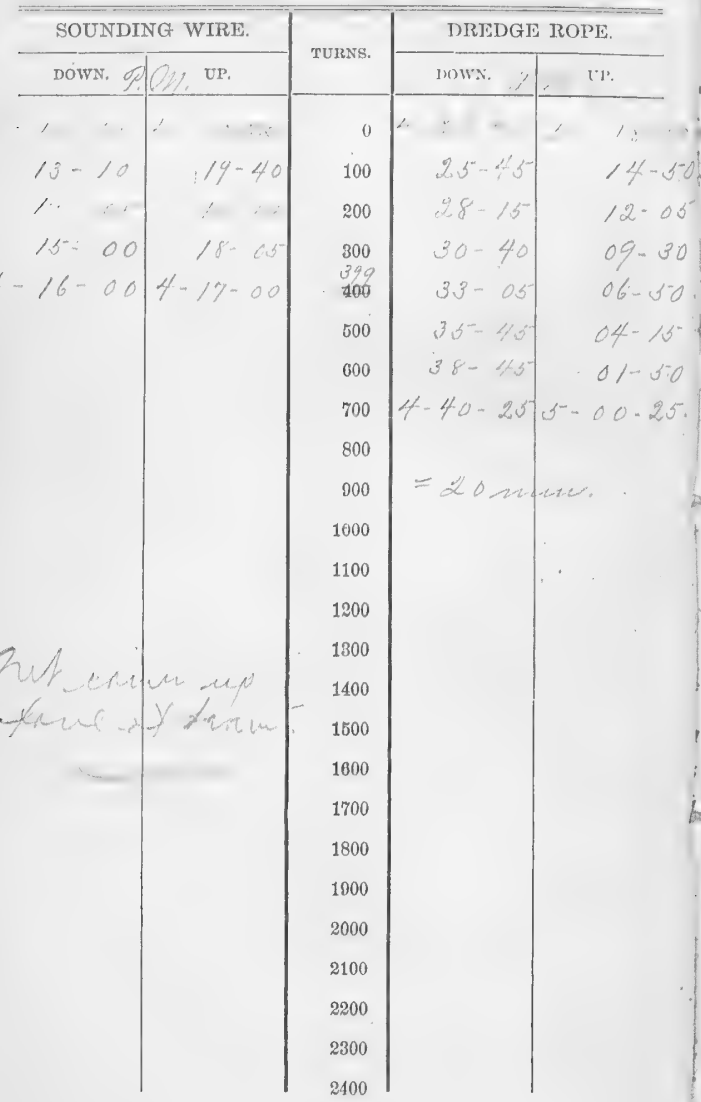




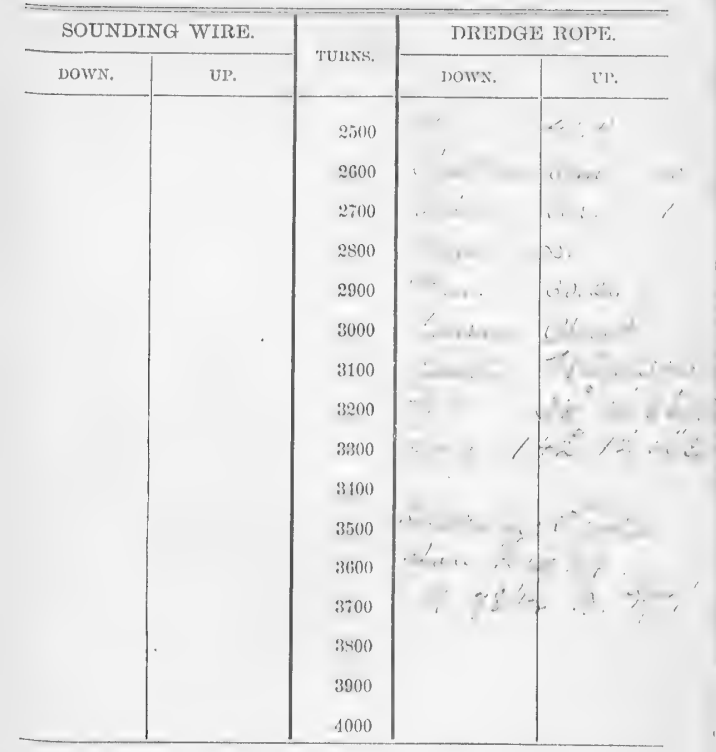

SERIAL TEMPPRATURES.

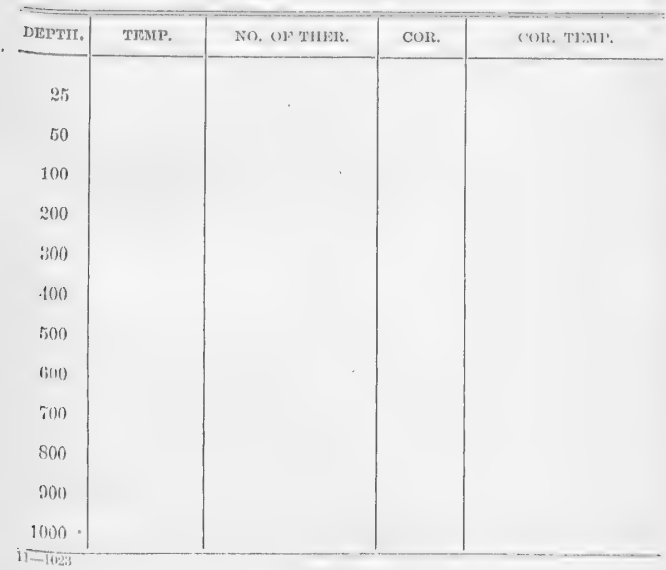

REMAIRTS: 
307 for

in:

No.

No. 10.

50,5 Date

Machine. Reel.
Turns
Corr.t;
Depth

Shot or lead.

Bottom

Bottom temperature

No, of thermometer

Cor.

Corrected temperature

Air S"y $^{\circ}$ ' Surface $62^{\circ}$ Drift

Trecul. or avertsic 2 lsts towimg a stern

Eilialit)

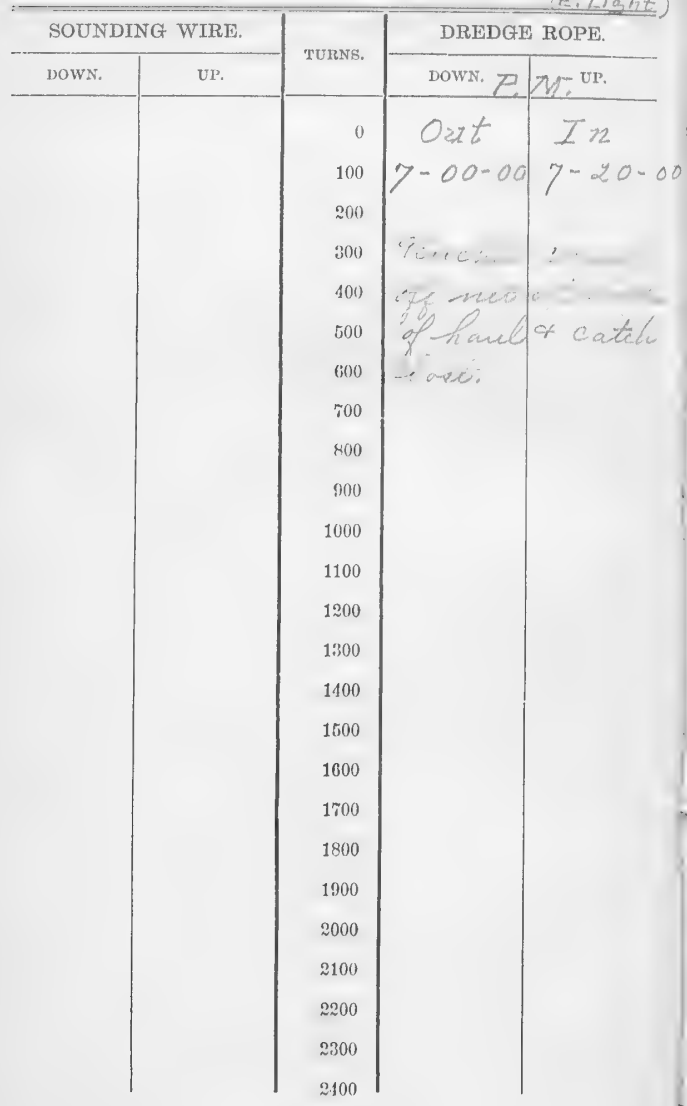




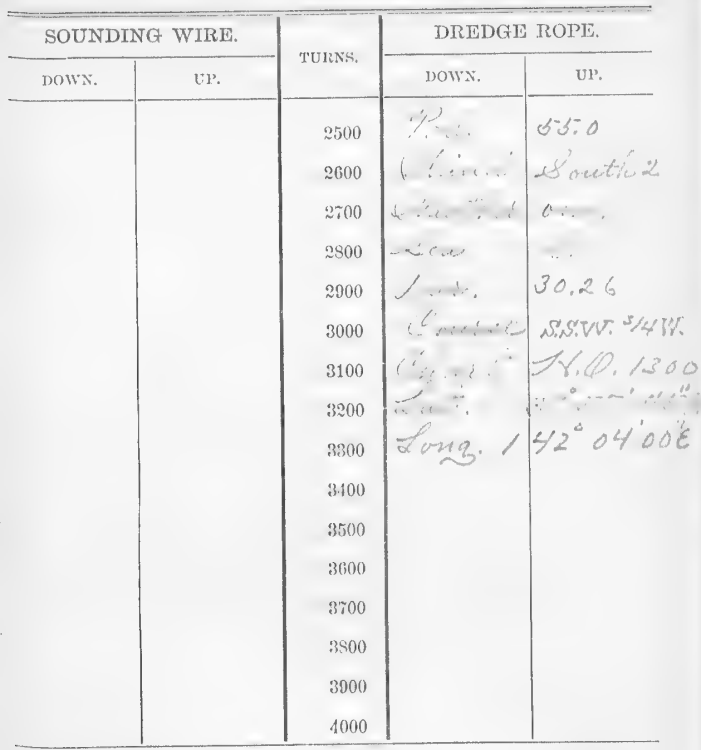

SWRIAI, TIEMPTRATURES,

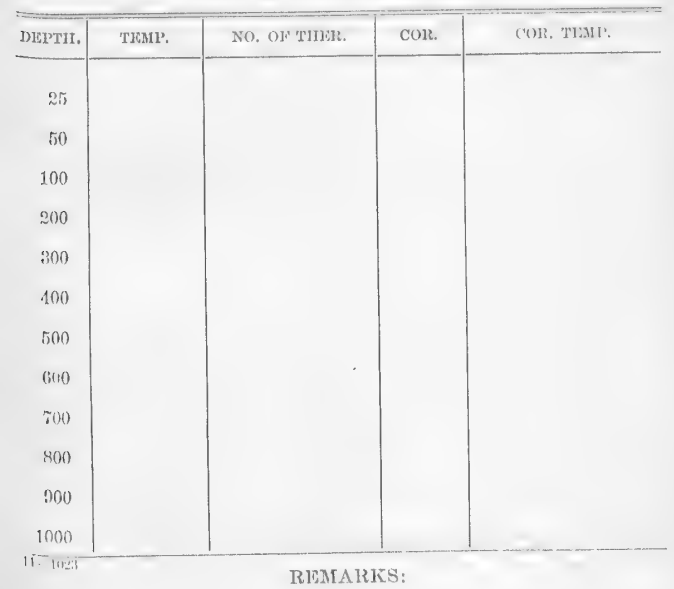


No. 10

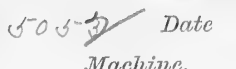

Machine.

Reel.

Tumis

Cor.t

Depth

Shot or lead

Bottom

Bottom temperature

No. of thermometer

Cor.

Corrected temperature

Air $57^{\circ}$ Surface $62^{\circ}$ Drift

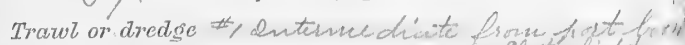
secitiol

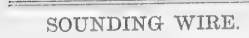

Dowa.
DREDGE ROPE.

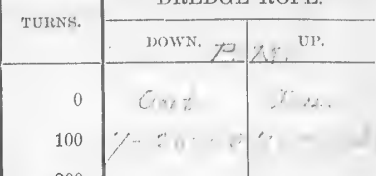


No.

D)rete

3 finerw Machine. Rect. Ameng

Turns Cor.t Depth 503 fmo.

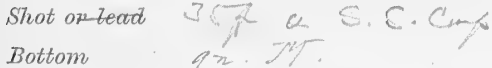

Bottom temperature

No. of thermometer $/ 08 / 53$. Cor. . . ,

Corrected temperature

3409

Air $66^{\circ}$ surface $7 \%^{\circ}$

Drift

Trawl or dredgo Ql

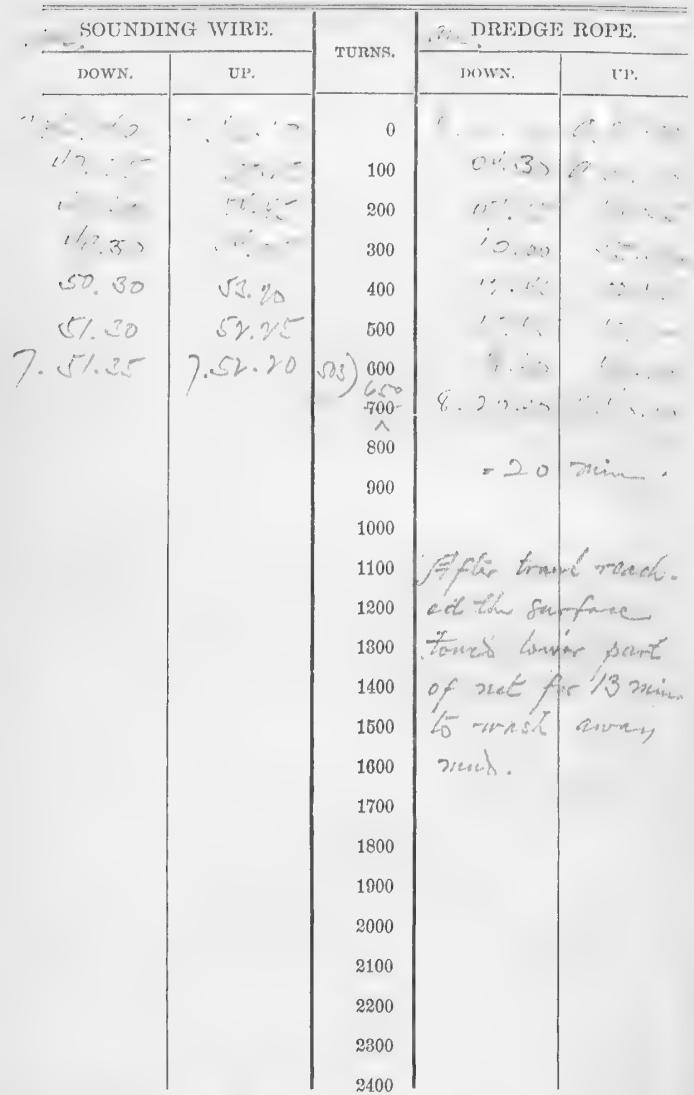


All

$i \sqrt{1} \%$

50.526

Date

12 Oet. 1906.

Dnex Machine. Recl. diven
Turns
Corit
Depth $282 /$ smo.

Shot or toad $3 \sqrt{x}$ \& S-s-Cms

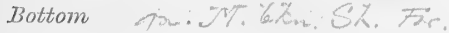

Bottom temperature

$4 c^{\circ}$

No. of thermometer $108155, \quad C o r ., 2$

Corrected temperaturc

Air $71^{\circ}$ Surface $74^{\circ}$ Drift

Trawt or aredge Q 'Ft5.. .

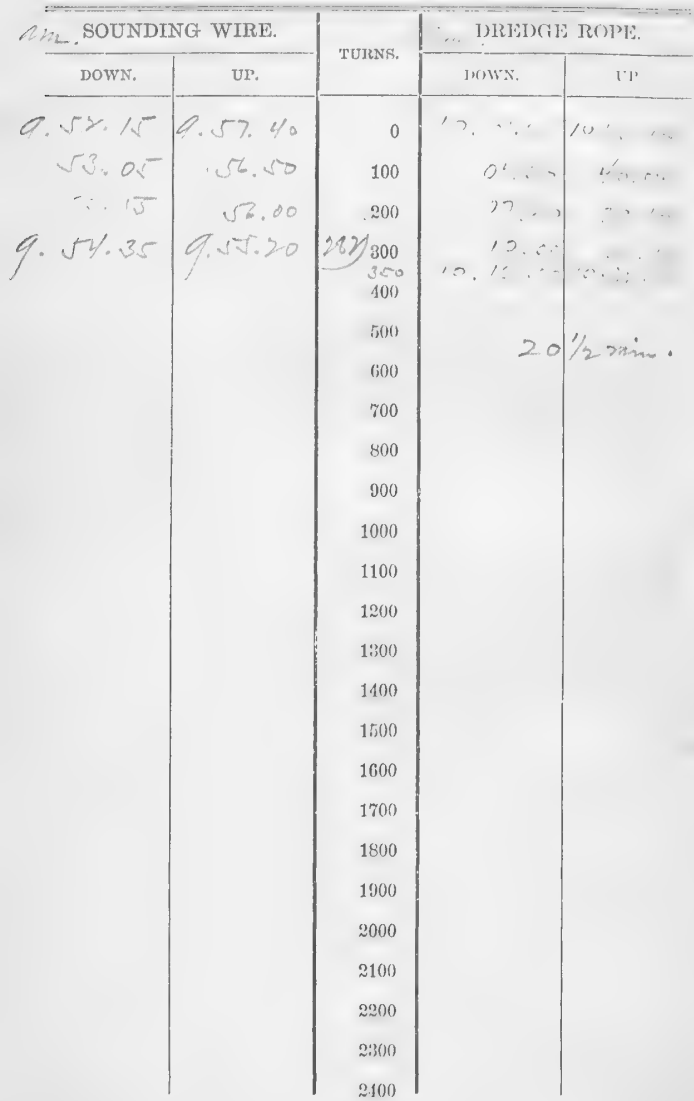


i से

QNo. D. To.5. Date 1.2 O.t. 1906.

$j$ Onero Machine. Reel. Lum.

Tums Cor.t Depth 124 fruo.

Shot ar lead B.F \& S. S. Cons:

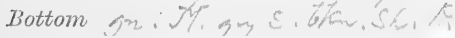

Bottom temperature SL:

No. of thermometer $108 \mathrm{k} \mathrm{s}^{2}$ Cor. _... 2

Corrected temperature $\quad$ o to ${ }^{\circ} \mathrm{C}$

Air $72^{\circ}$ Surface $7 y^{\circ}$ Drift

Trawl or dredge of flibeti-ass-132nice.

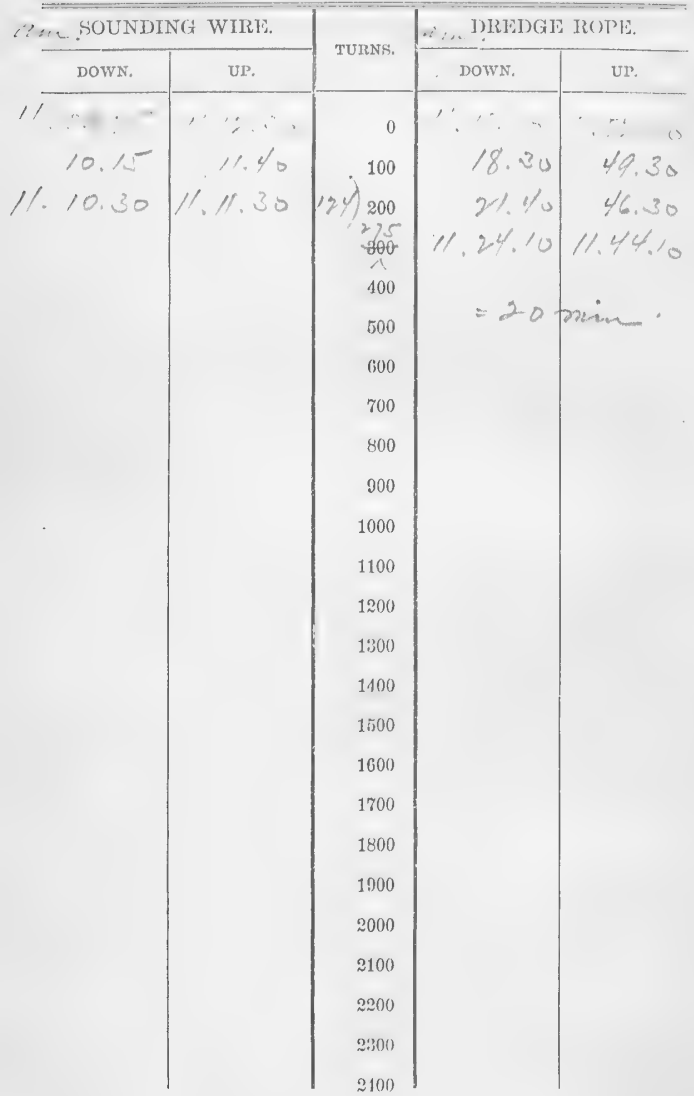


No Date'

HC \& Machine Reel.
Turns
Cor.t
Depth

Shot or lead

Bottom Cl, )

Bottom/temperature

No. of thermometer $/ C$ Cor. 2

Corrected temperature

Air , 1 Surface

Drift

Trawt or dredge

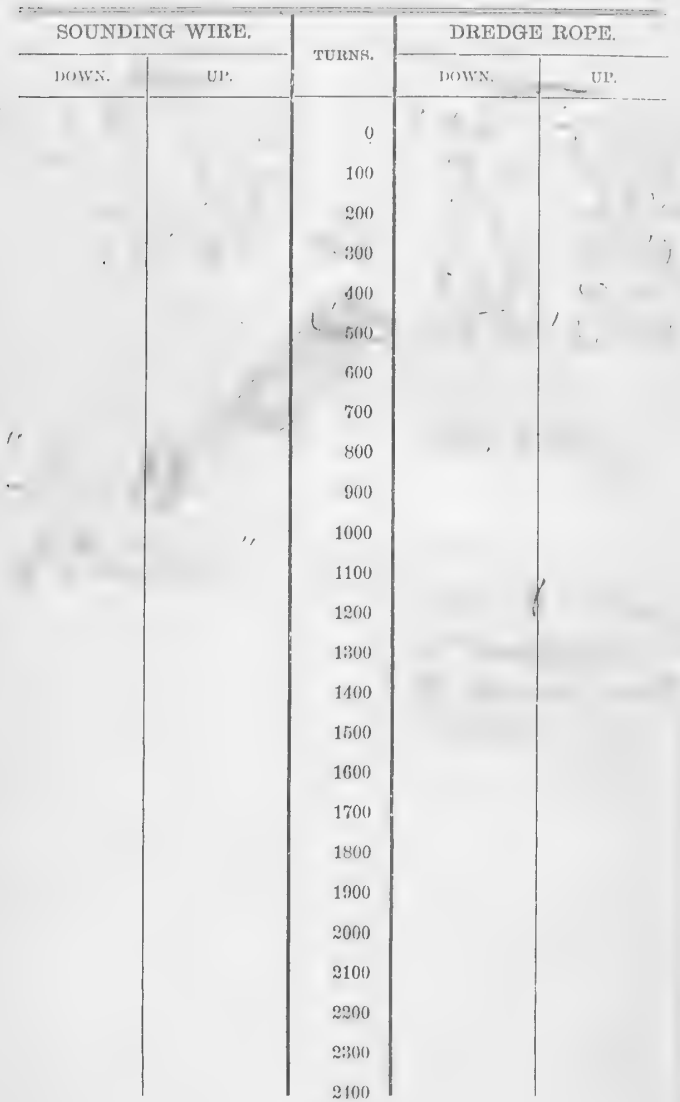


No. 77 of

Date $/ y$

Lat Machine. Reel.

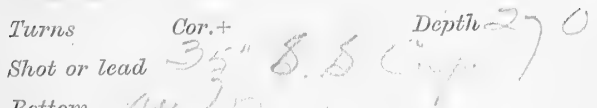

Bottom

Bottom temperature

No, of thermometer

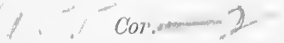

Corrected temperature

Air $>$ Surface 73 Drift

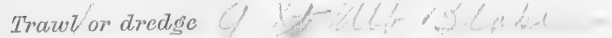

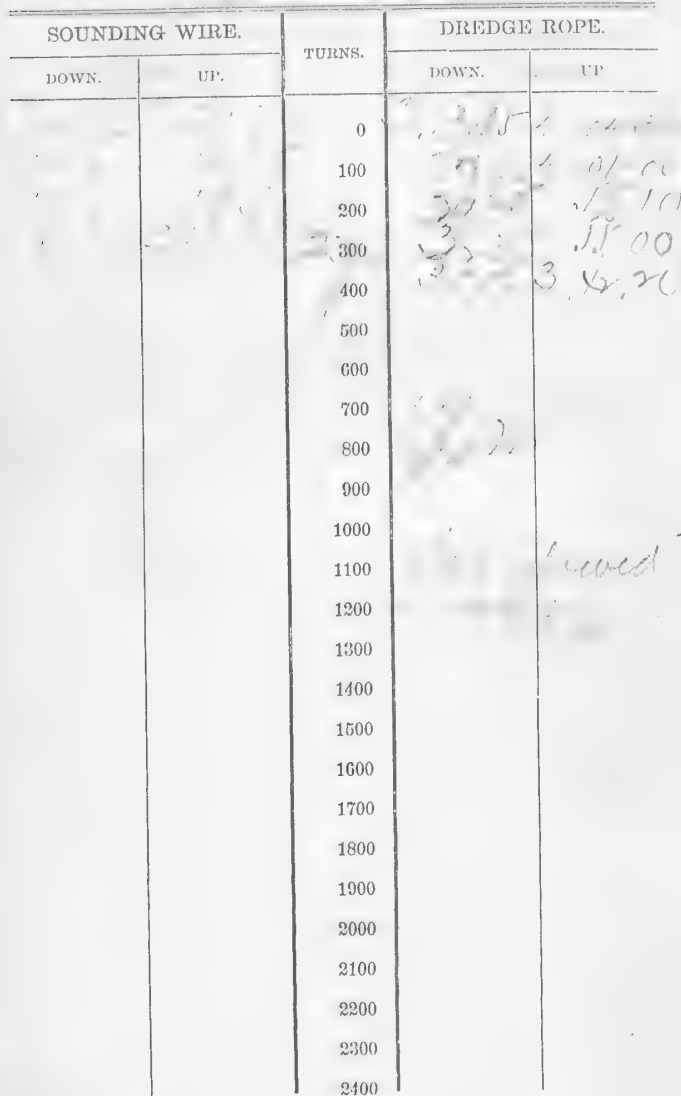




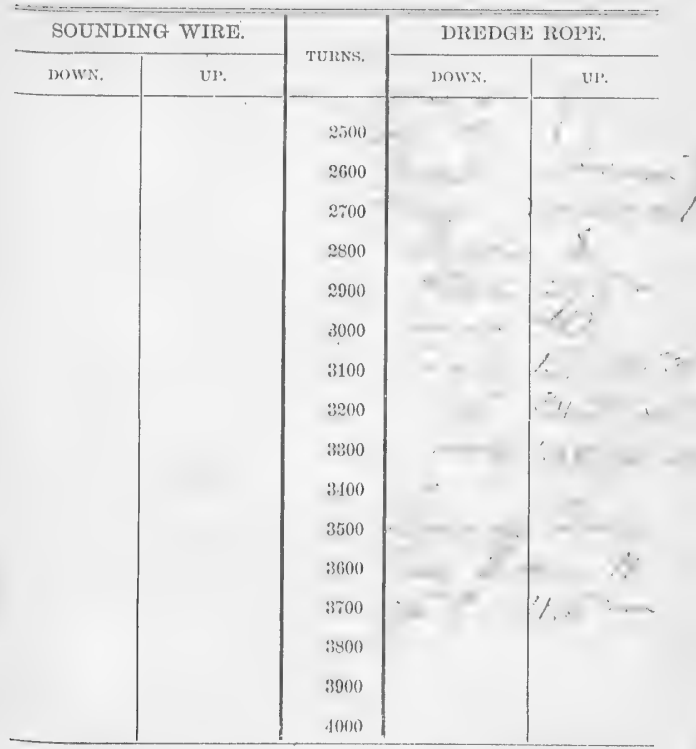

SERIAL TEMPERATURES

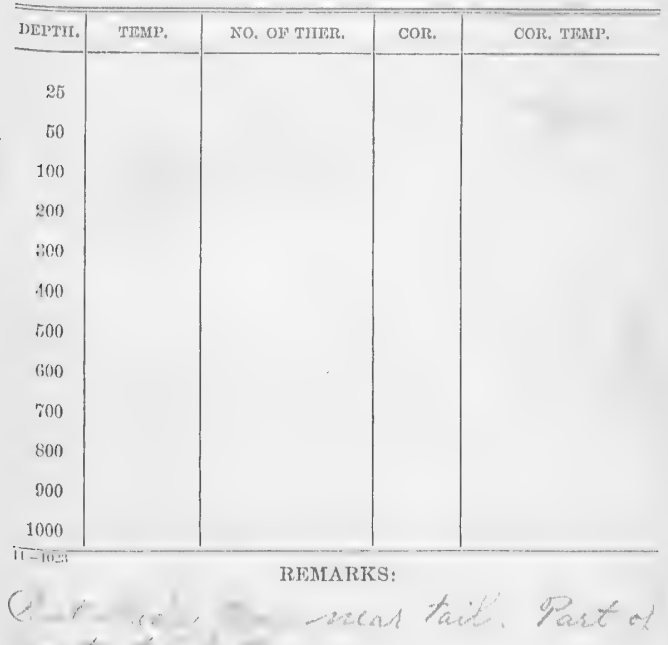


No. WO W-8 Date Qet, 310

Machine. Reel.

Turns Cor.t Depth

Shot or lead

Bottom

$-10$

Bottom temperature

No. of thermometer.

as, cor.

Corrected temperature

Air $63^{\circ} \quad$ Surface $70^{\circ}$ Drift

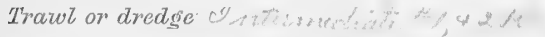

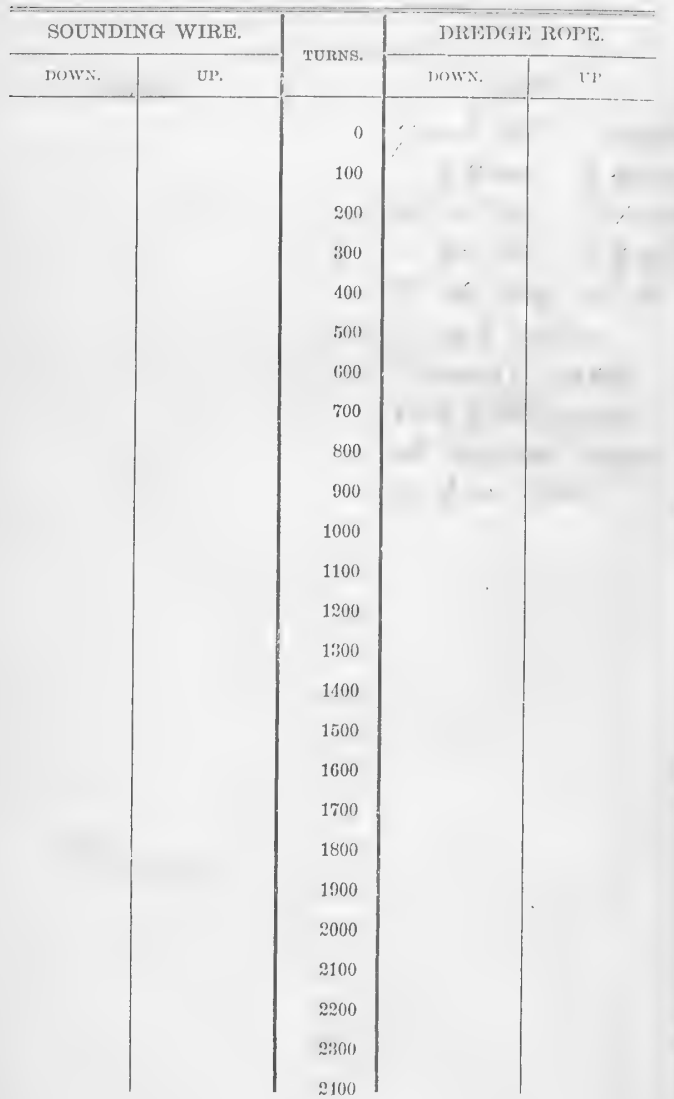




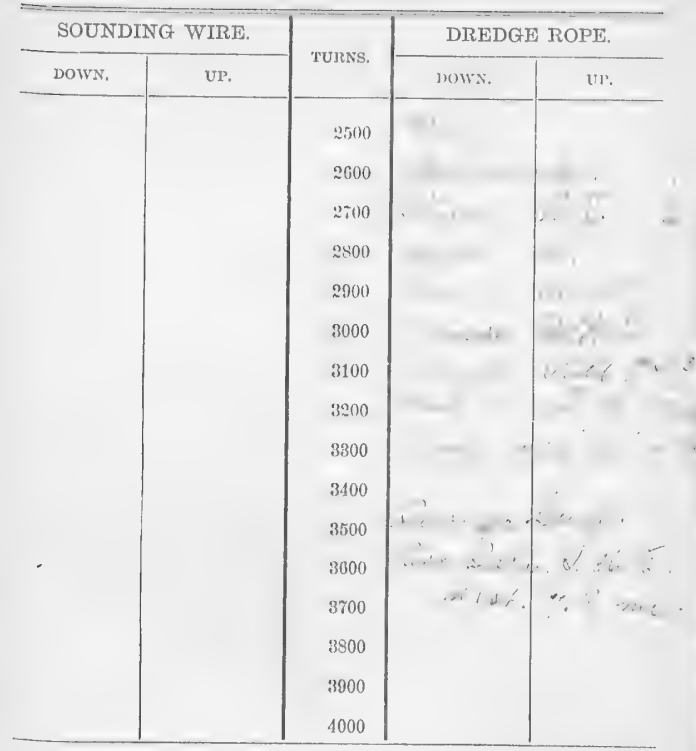

SERIAL TEMPERATURES.

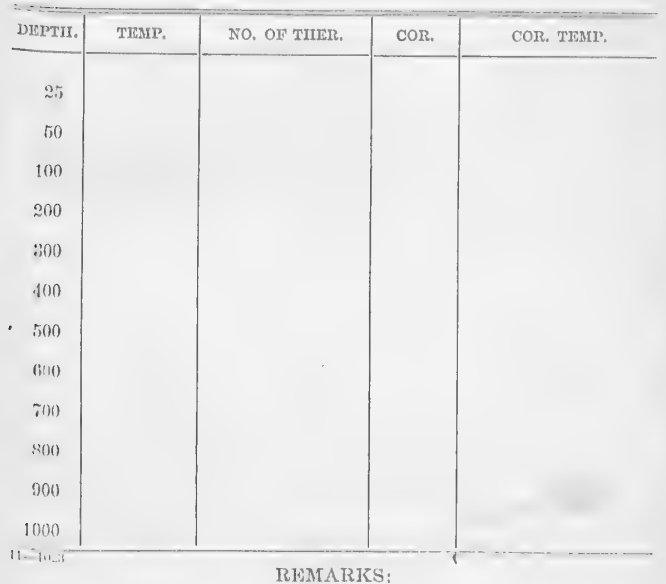





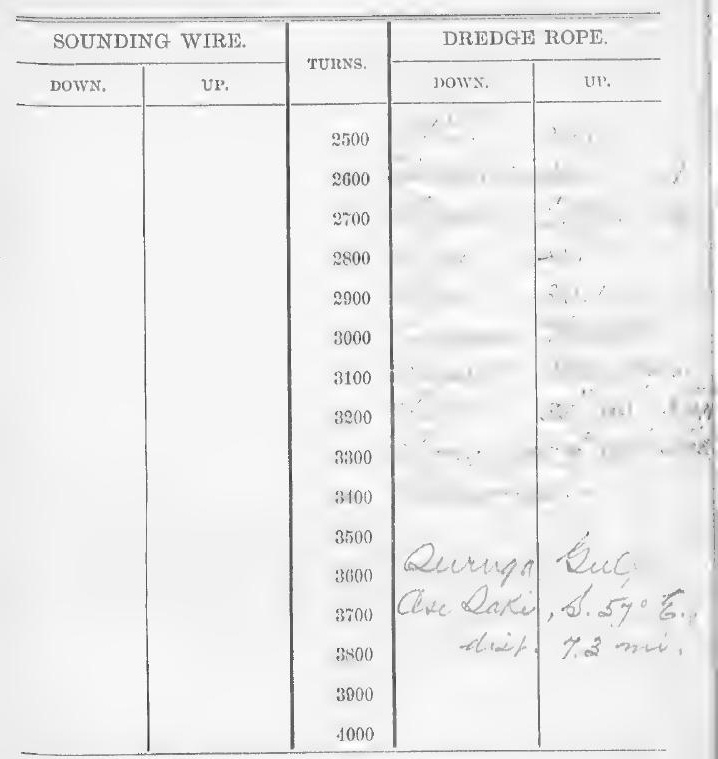

SERIAL TEMTPERATURES.

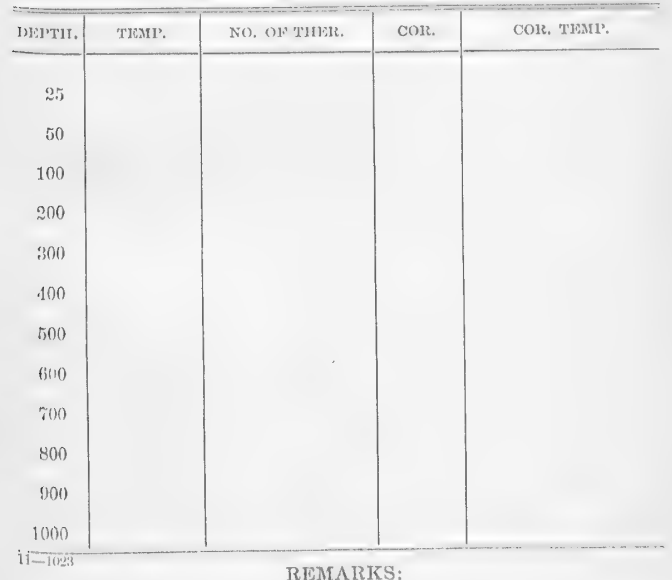

REMARKS: 


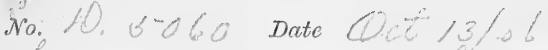

-Lucal Machine Reel. Inecres

Turns Cor.t Depth 197

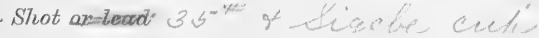

Bottom ess. bla. S.

Bottom temperature $\checkmark-0.8^{-6}$

No. of thermometer / o \& / f o Cor. .

Corrected temperature of $0.6^{6}$

Air $66^{\circ} \quad$ Surface $70^{\circ}$ Drift

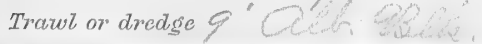

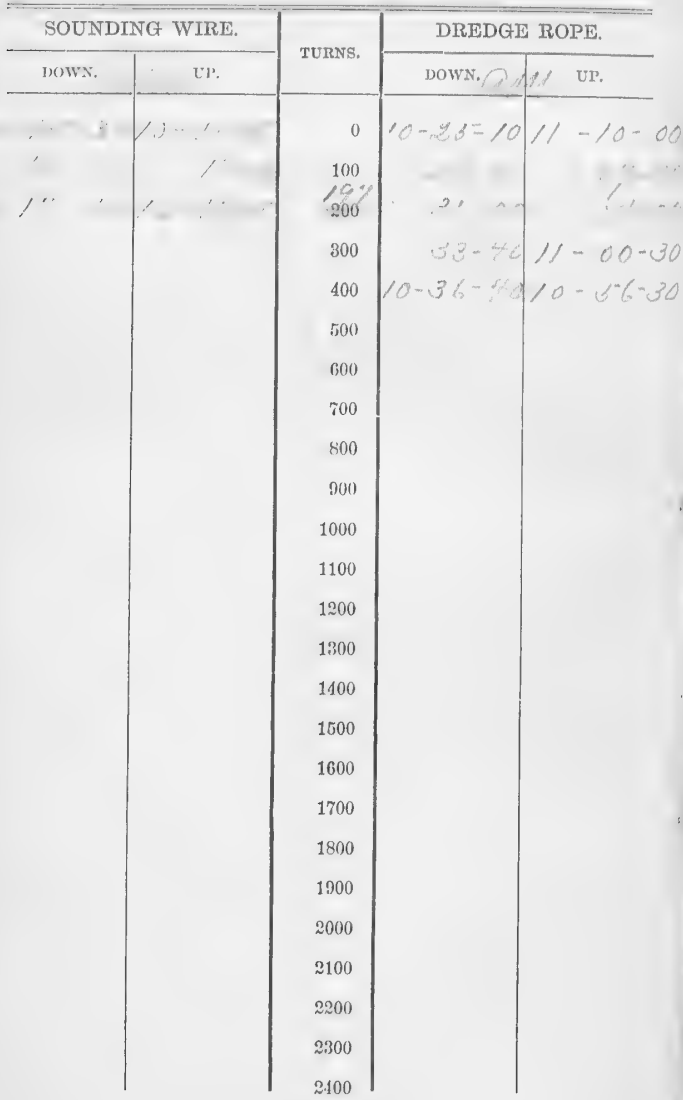




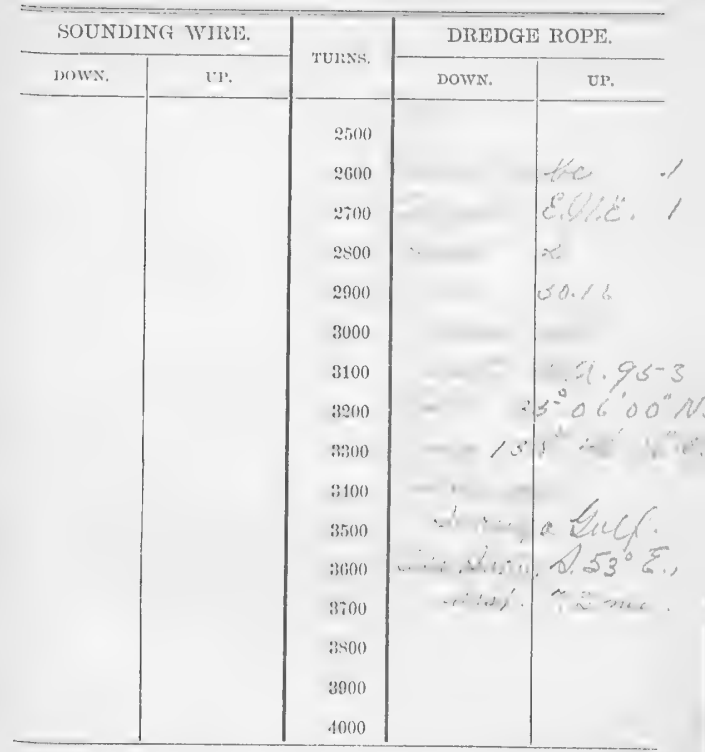

SERIAL TEMPERATURES,

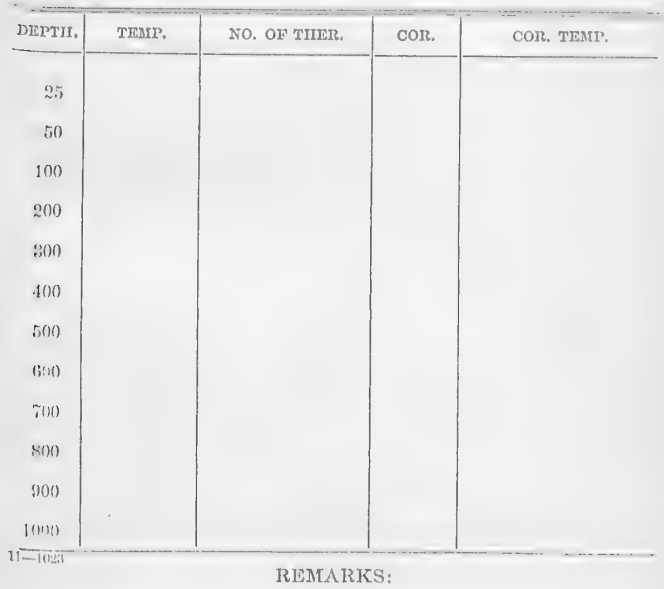




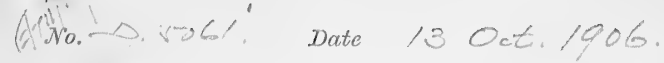

\section{- Zuense Machine, Reel. Zucens}
Turns
Cor.t
Depth 832 Fro.

Shat on-lead 35 m m. S.S.Cics.

Bottom

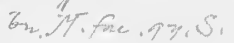

Bottom temperature

$$
43: 9
$$

No. of thermometer $108 / \sqrt{ }$. Cor. _. . 2

Corrected temperature 4/3:?
Air $68^{\circ}$
Surface
Drift

Trawt or dredge

T'STE. - MZZE.

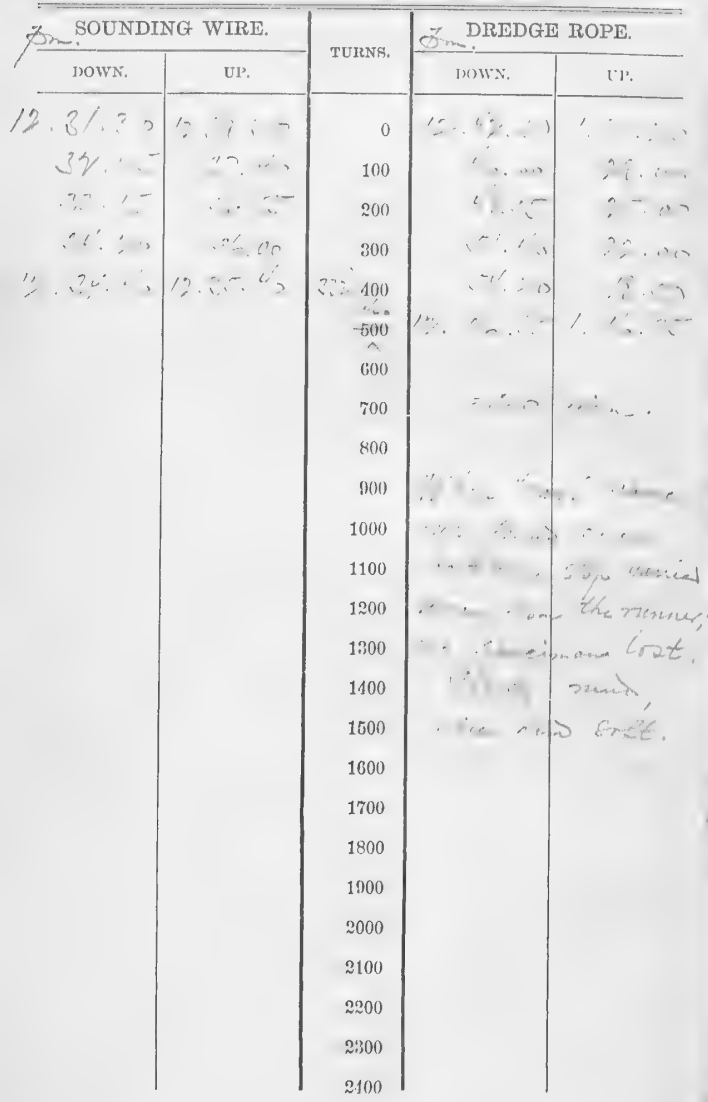




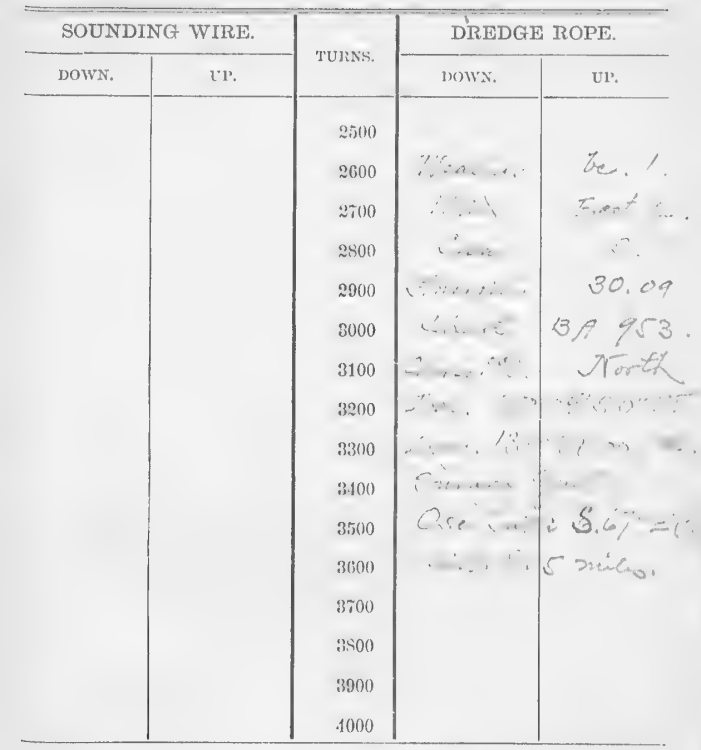

SERIAL TEMPERATURES.

\begin{tabular}{|c|c|c|c|c|}
\hline DIPTH, & тнми'. & No, of 'THIR. & COIR. & COR. 'TसML', \\
\hline 25 & & & & \\
\hline 50 & & & & \\
\hline 100 & & & & \\
\hline 200 & & & & \\
\hline 300 & & & & \\
\hline .100 & & & & \\
\hline 500 & & & & \\
\hline (6in) & & & & \\
\hline 700 & & & & \\
\hline 800 & & & & \\
\hline 900 & & & & \\
\hline 1000 & & & & \\
\hline
\end{tabular}

REMARKS: 
No. D. 5062 Date 13 oct. 1906. ) Rueno Machine. Reel Lumaro
Turns
Cor.+
Depth
250 fino.

Shot on teat $3.5,4$ \&. S.S.C.n.

Bottom

Bottom temperature $\quad$ / 7.9

No. of thermometer $108 / \mathrm{sJ}^{-}$Cor. …2.

Corrected temperature $\quad \% / 7: ?$

Air: $77^{\circ} \quad$ Surface $72^{\circ}$ Drift

Trawt or dredge o' Flb. - BLAe.

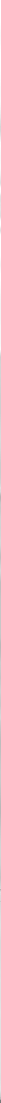




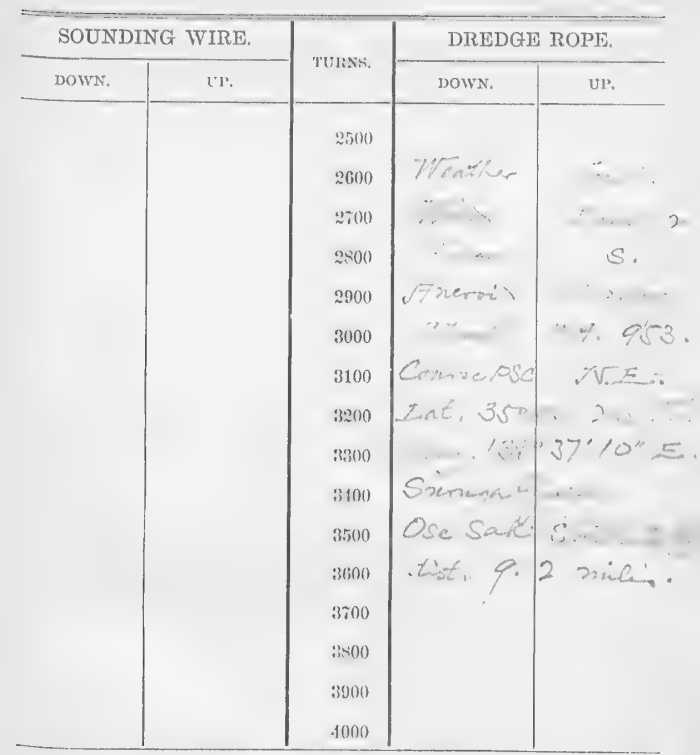

SERIAL TEMPERATURES.

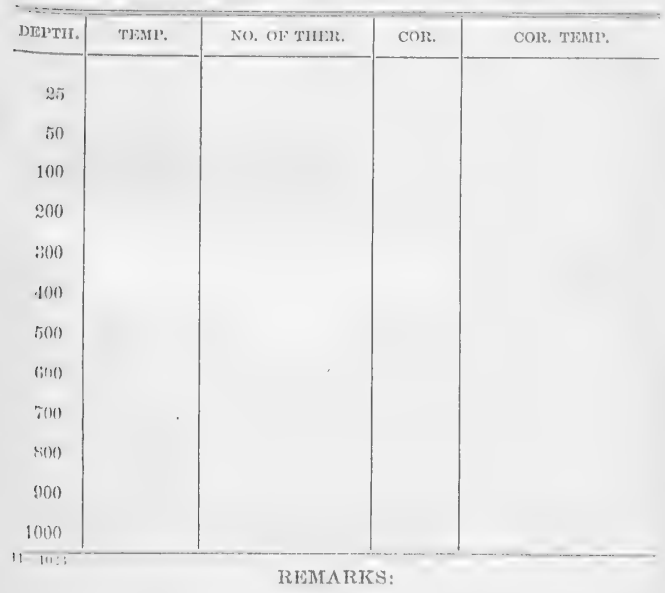


No. D. 5060

Date

13 oct 1906. Machine, Reel.

$\jmath$
Turns
Cor.+
Depth

Shot or lead

Bottom

Tro somentime.

Bottom temperature

No. of thermometer.

Cor.

Corrected temperature
Ait
Surface
Drift

Trawt or dredse

\begin{tabular}{|c|c|c|c|c|}
\hline \multicolumn{2}{|c|}{ SOUNDING WIRE. } & \multirow{2}{*}{ TURNS. } & \multicolumn{2}{|c|}{$\because$ DREDGE ROPE. } \\
\hline DOWN. & UP. & & Down. & vp. \\
\hline & & $\begin{array}{r}0 \\
100 \\
200 \\
300 \\
400 \\
500 \\
600 \\
700 \\
800 \\
900 \\
1000 \\
1100 \\
1200 \\
1300 \\
1400 \\
1500 \\
1600 \\
1700 \\
1800 \\
1900 \\
2000 \\
2100 \\
2200 \\
2300 \\
2400\end{array}$ & $\begin{array}{c}3.12 .15 \\
1 . \\
3 \vdots \\
3 \ldots \\
\text { ramed } 30 \\
\therefore \ldots\end{array}$ & 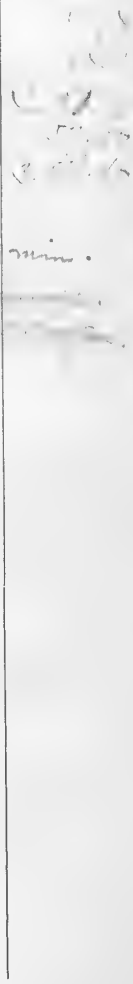 \\
\hline
\end{tabular}




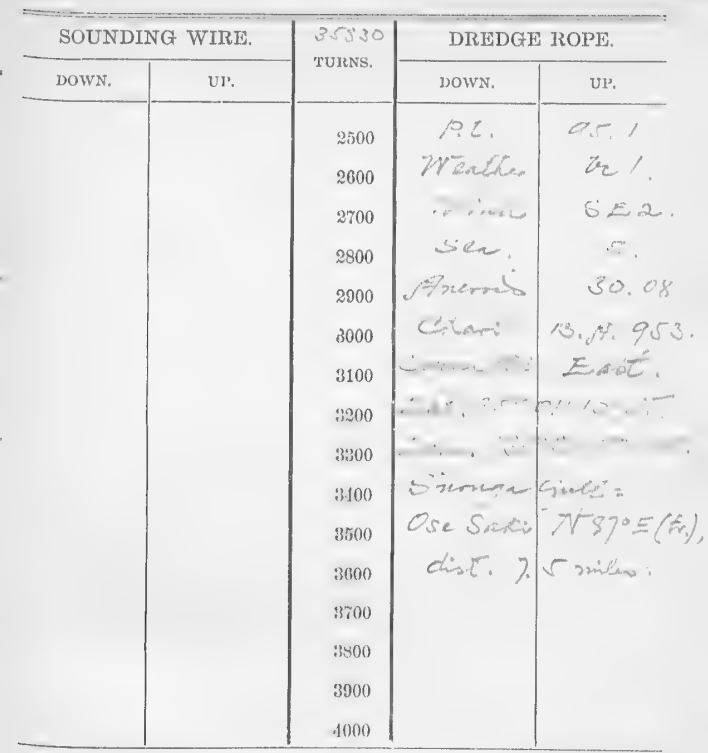

SERIAL TEMPERATURES

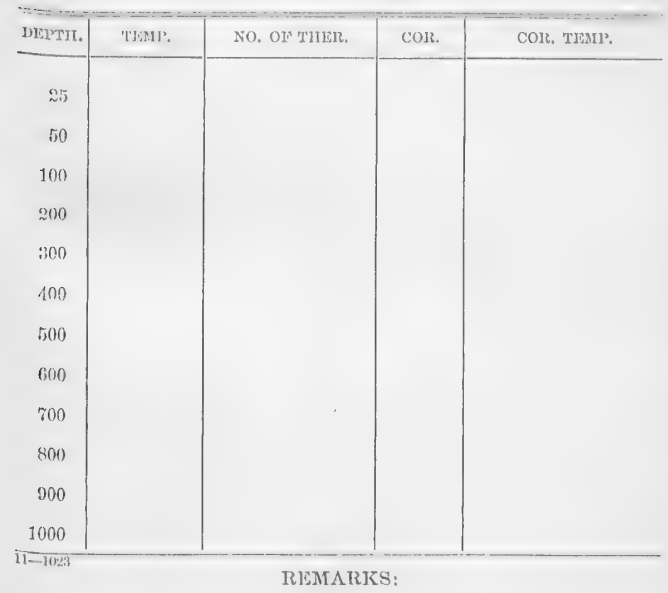


No.?

a $8:-$

Date 15

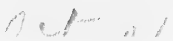

Machine, Reel.
Turns
Cor.+
Depth

Shot or lead

\section{Bottom}

\section{Bottom temperature}

No. of thermometer

Cor.

Corrected temperature
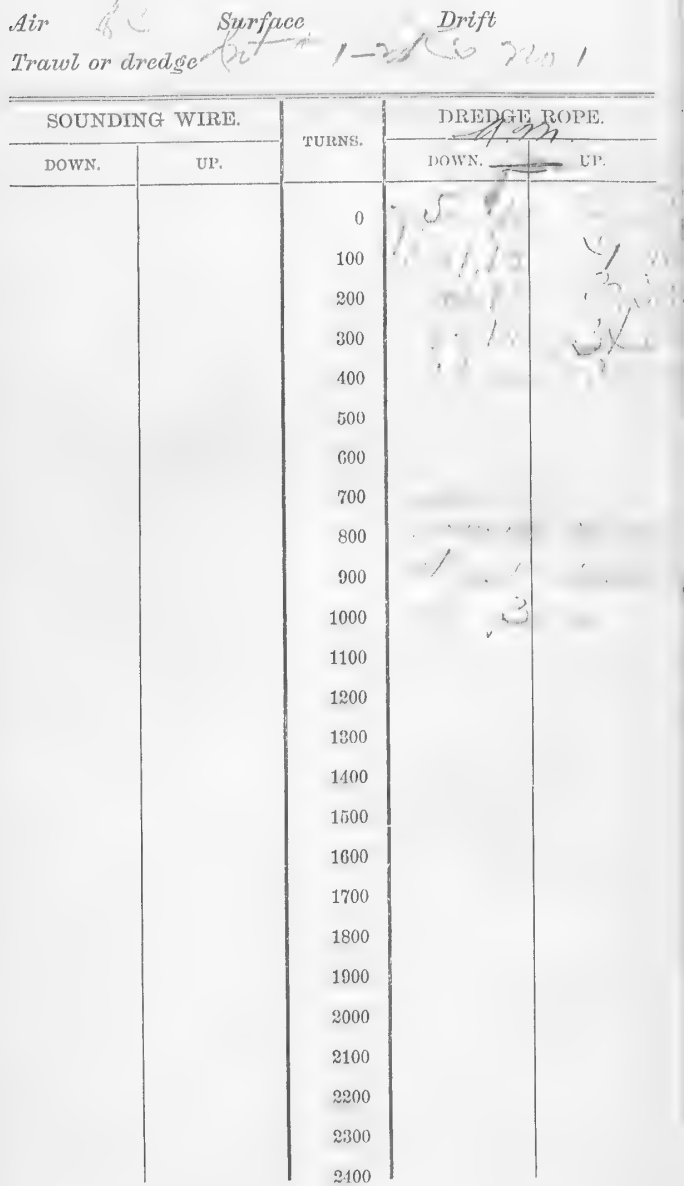


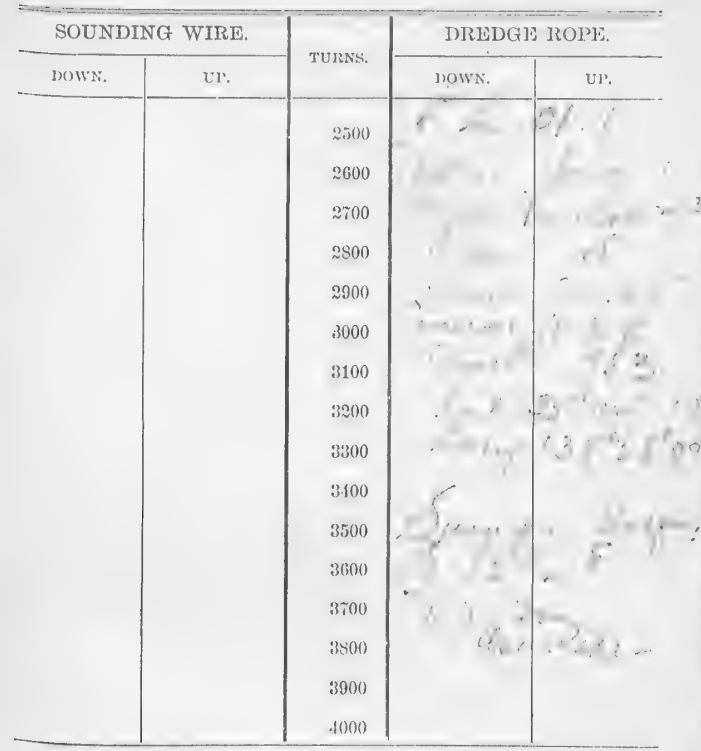

SLIRIAL, TEMIPERATURES

\begin{tabular}{|c|c|c|c|c|}
\hline Iи:тTI. & туМ⿻:. & No. OW THuEr. & cols. & COR, тL:LI:. \\
\hline 25 & & & & \\
\hline 50 & & & & \\
\hline 100 & & & & \\
\hline 200 & & & & \\
\hline 300 & & & & \\
\hline .100 & & & & \\
\hline 500 & & & & \\
\hline $\operatorname{lin} 0$ & & & & \\
\hline 700 & & & & \\
\hline 800 & & & & \\
\hline 900 & & & & \\
\hline 101000 & & & & \\
\hline
\end{tabular}

RWMARKS: 
No

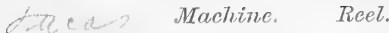

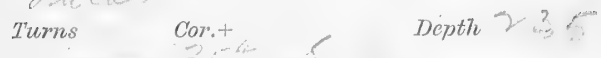

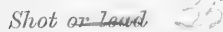

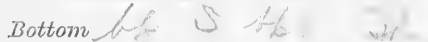

Bottorn temperature

No. of thermometer

Cor. ...

Corrected temperature

Air Surface * Drift

Trawl or dredge, of $\therefore$.

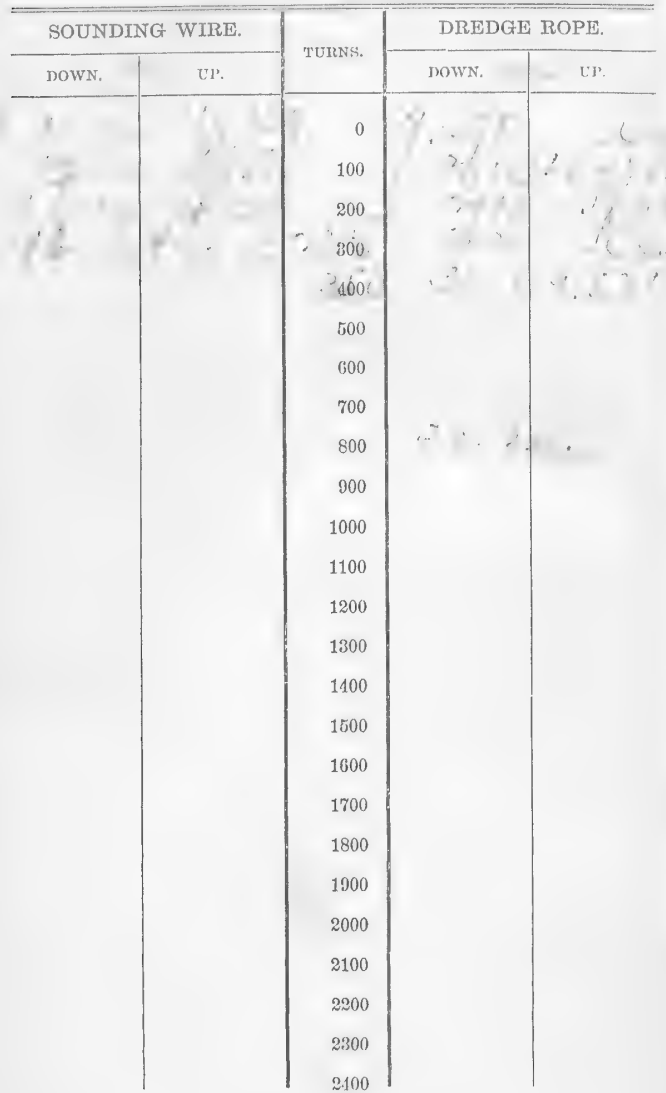




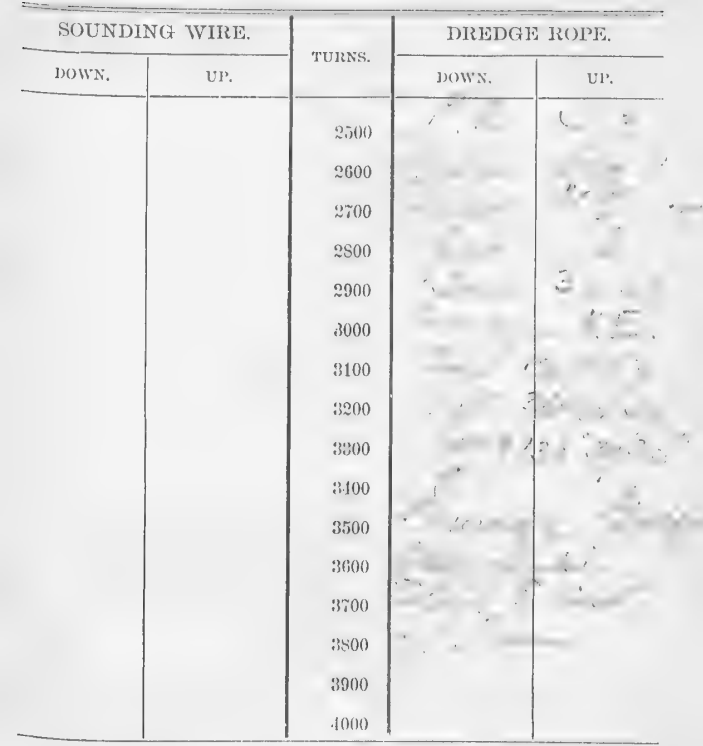

SEIRIAL TIEMIPRATURES

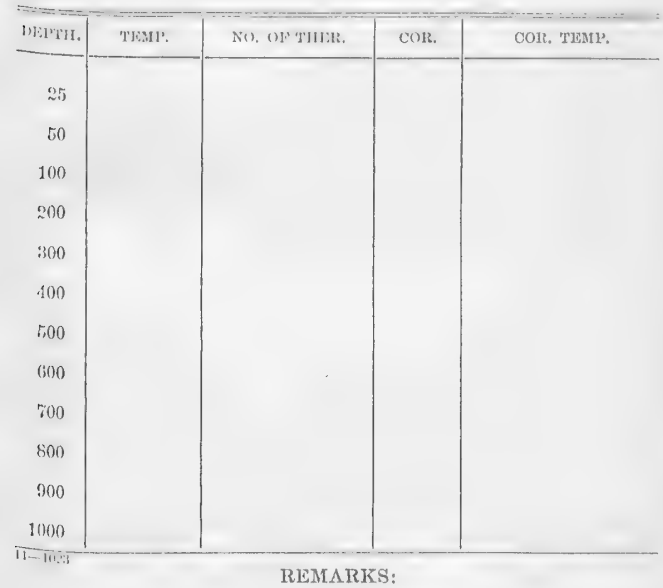




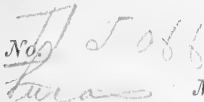

Machine. Reel.

Turns Cor.t of Depth

Shot pindeach $=$

Bottom fre. $6 \pi .1$

Jottom temperature

No. of thermometer

5

Corrected temperature of 0.8

Air 74 Surface 72 Drift

Trawt or aredge $\quad q^{\prime}$ औ

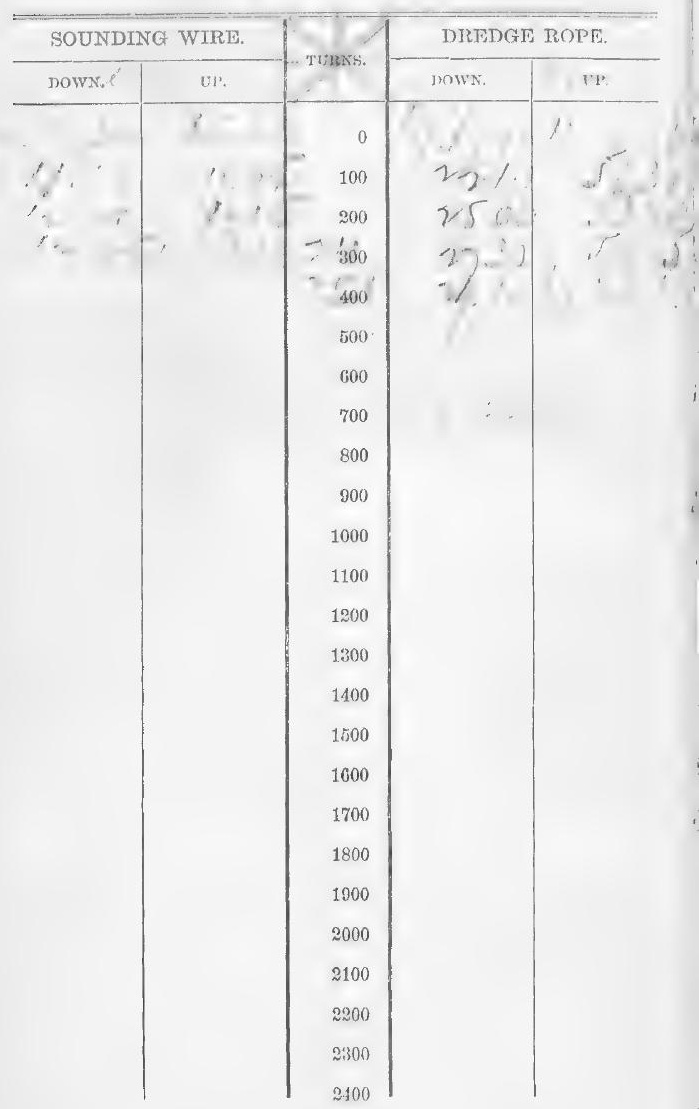




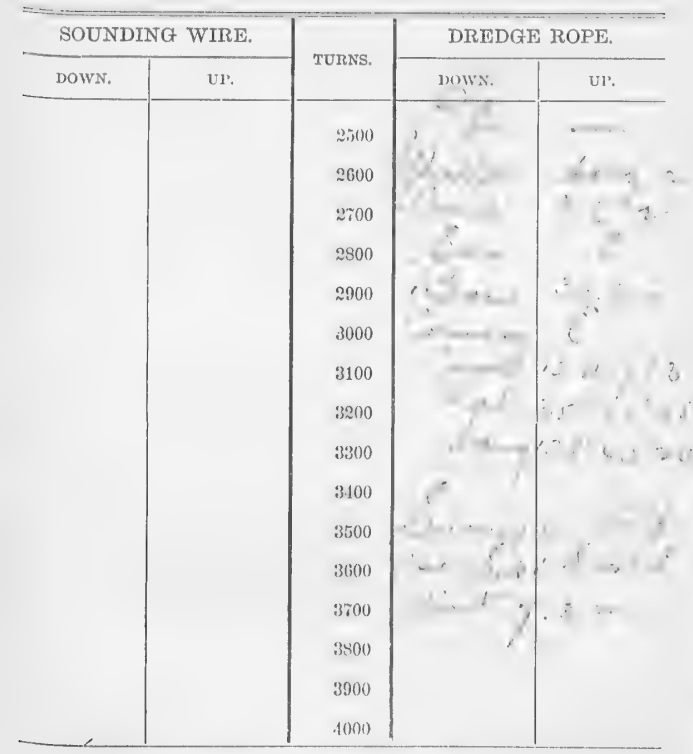

SFRIAL TIEMPERATUIRES

\begin{tabular}{|c|c|c|c|c|}
\hline DEPTиI. & TIRMP. & NO. OF THUR. & corr. & Cor. TKMrr. \\
\hline 25 & & & & \\
\hline 50 & & & & \\
\hline 100 & & & & \\
\hline 200 & & & & \\
\hline 300 & & & & \\
\hline 400 & & & & \\
\hline 500 & & & & \\
\hline 600 & & & & \\
\hline 700 & & & & , \\
\hline 800 & & & & \\
\hline 900 & & & & \\
\hline 1000 & & & & \\
\hline
\end{tabular}




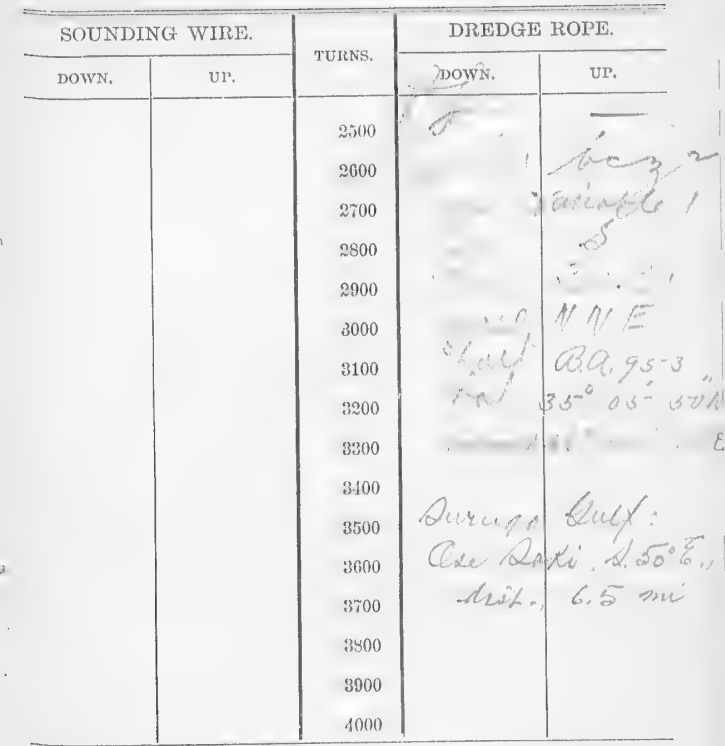

SERIAL TEMPERA'TURES.

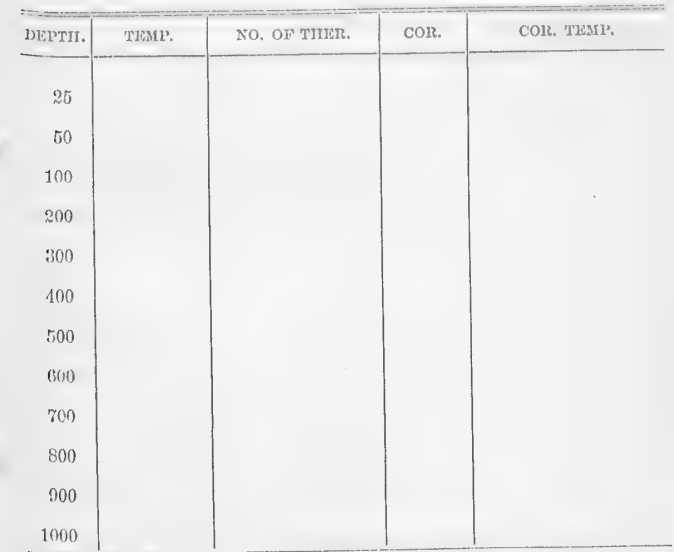

I1 1003

REMARKS: 
$i, 8$

No. 10 . Lucae

Date

Oet $16-1906$ Machine.

Rect. Lueat

Turns

$$
\text { Cor .+ }
$$

Depth

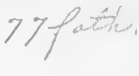

Shot or teat $3 v^{-*}$ \% segute

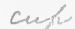

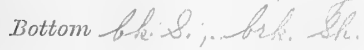

Bottom temperature $63.2^{\circ}$

No, of thermometer $10816-5-$

Cor: - -

Corrected temperature 63 "

Air $80^{\circ}$ Surface $72^{\circ}$ Drift

Trawl or dredge $/ 2^{\prime}$ प) a

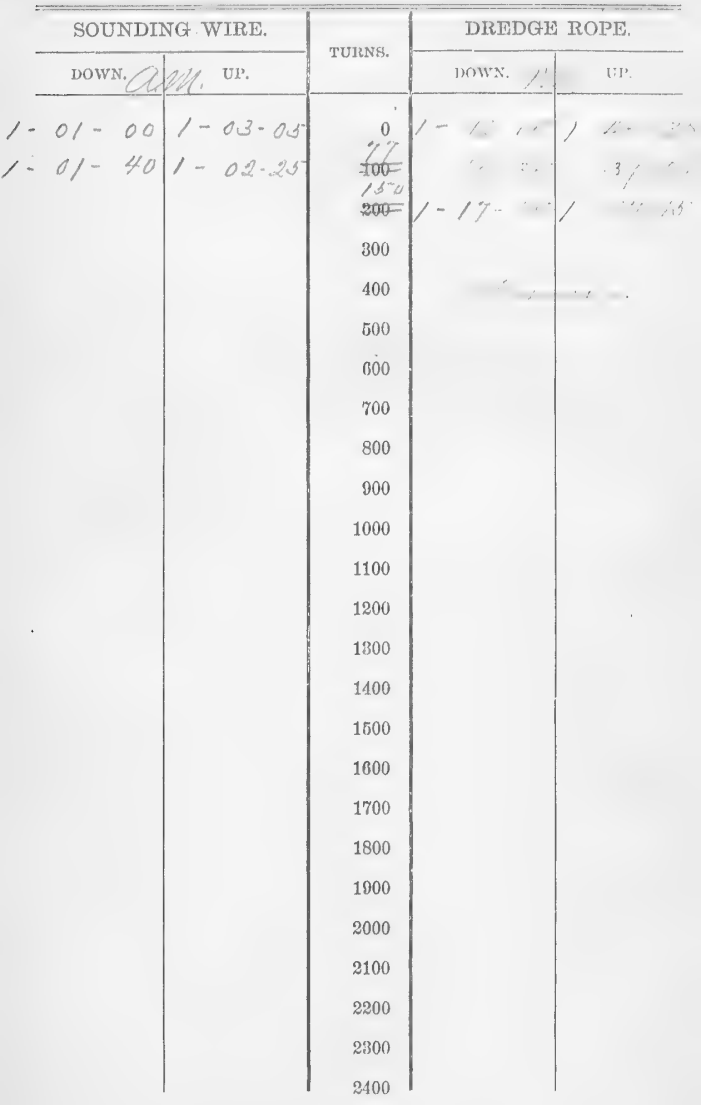




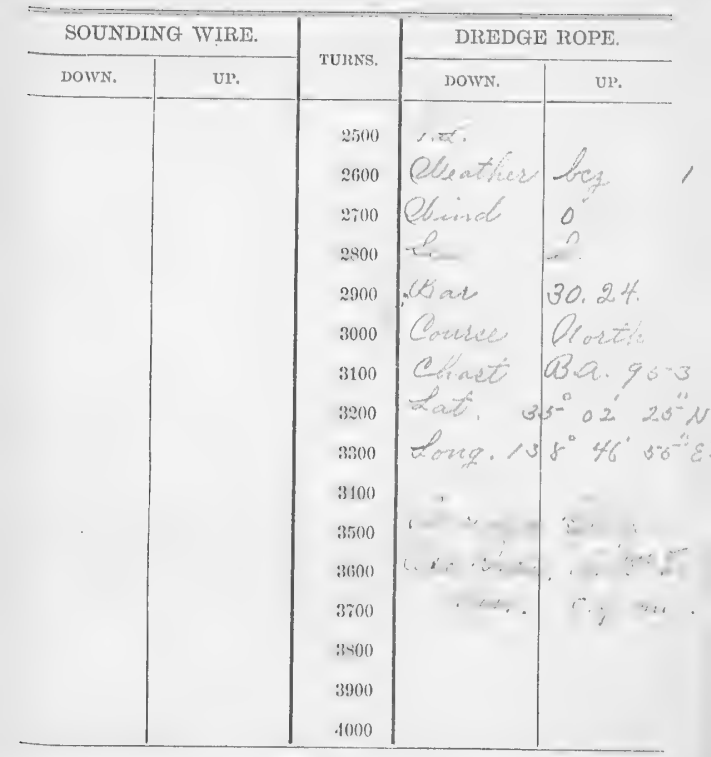

SERIAL, TEMPERATURES.

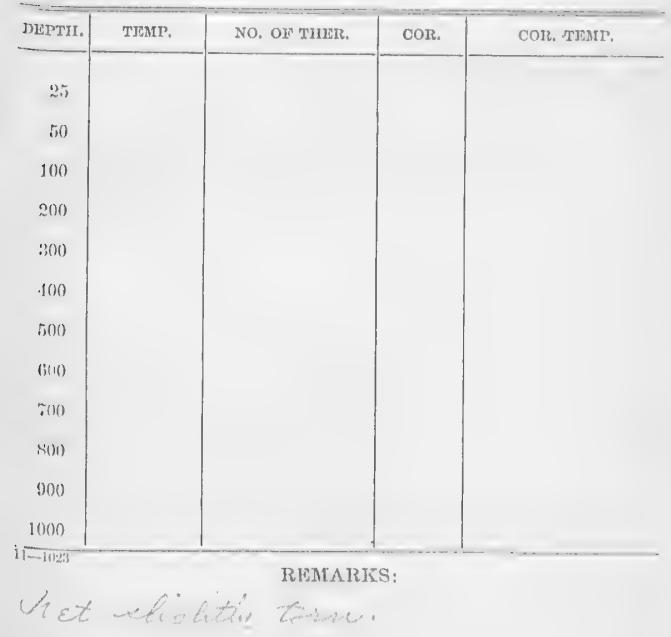


Xro.

Date Uat, 13,-1904

truenes Machine.

Recl. Leecrat

Turns Cor: +

Depth/3/ farth.

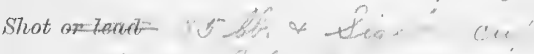

Bottom Ni, is, live de.'

Bottom temperature $5-6.0^{\circ}$

No. of thermometer $10 \mathrm{~S} / \mathrm{S}^{-3}$

Cor.

Corrected temperature

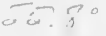

Air $8 \%$ Surface $73^{\circ}$ Irift

Trawt or dredge o' "isacera

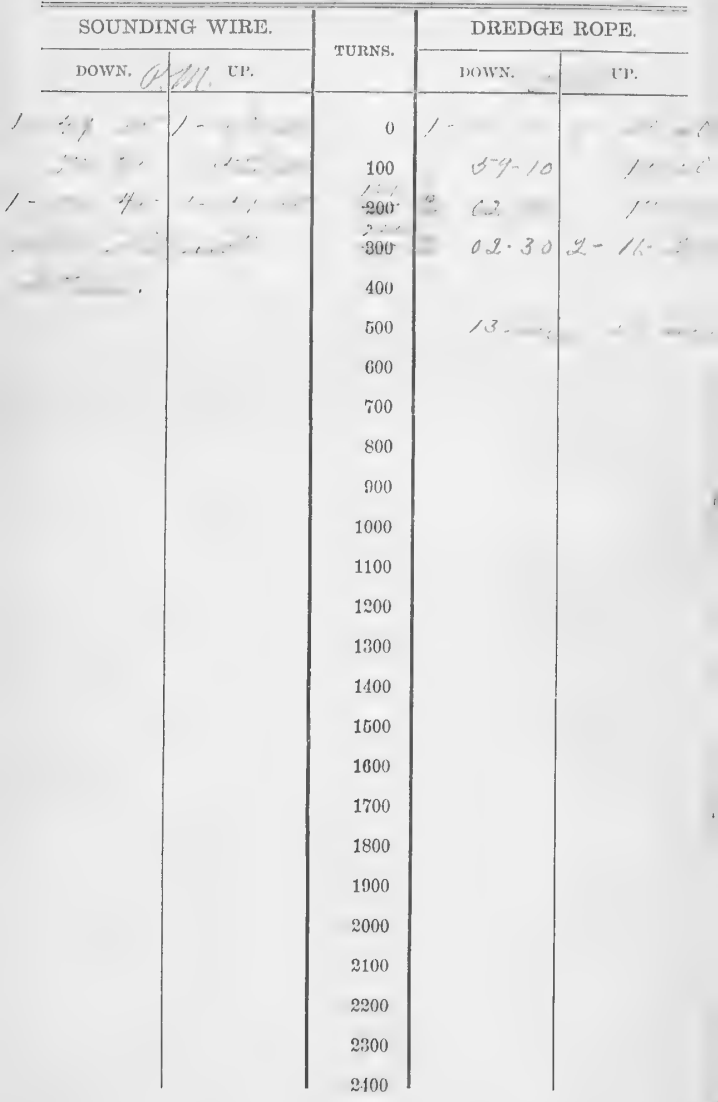


i

No. 10 .

5070

Date

40 ats; 1906

Luevos Machine.

Recl. Lucae

Turns

Cor.t

Depth 108 . oth

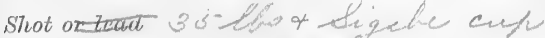

Bottom Dif, is, -12.5

Bottom temperature $5-7.8^{\circ}$

No. of thermometer $10815-5$ Cor.

Corrected temperature $5 \%, 6^{\circ}$

Air $88^{\circ}$ Surface $73^{\circ}$ Drift

Trawl or dredge 8 .

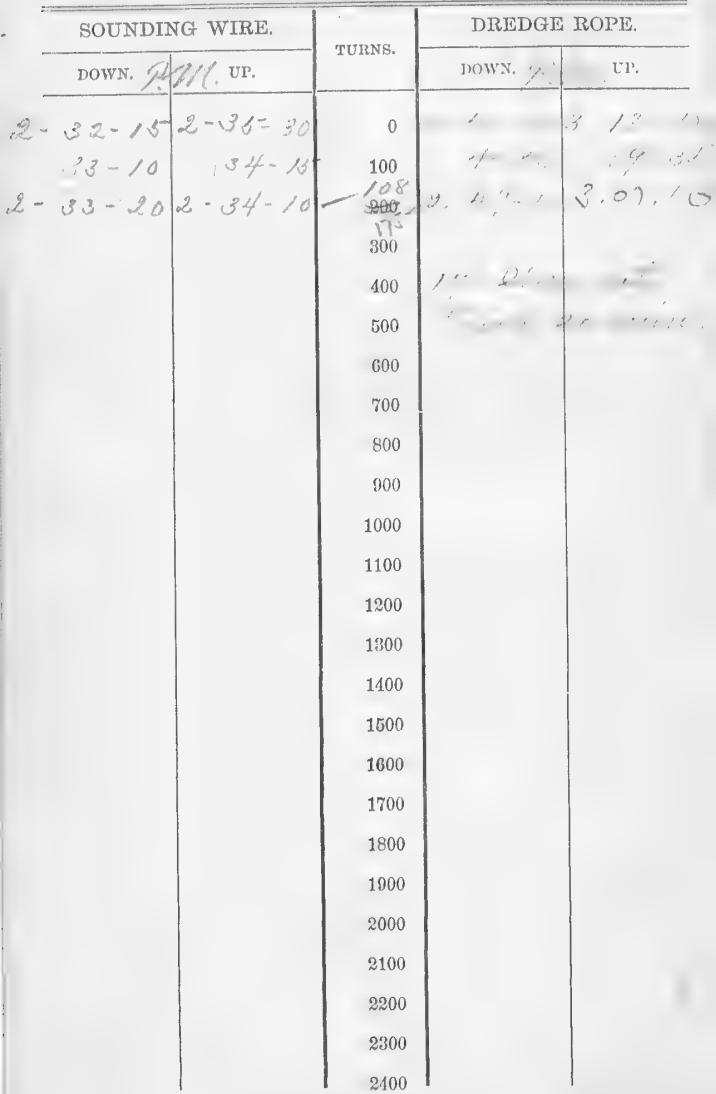




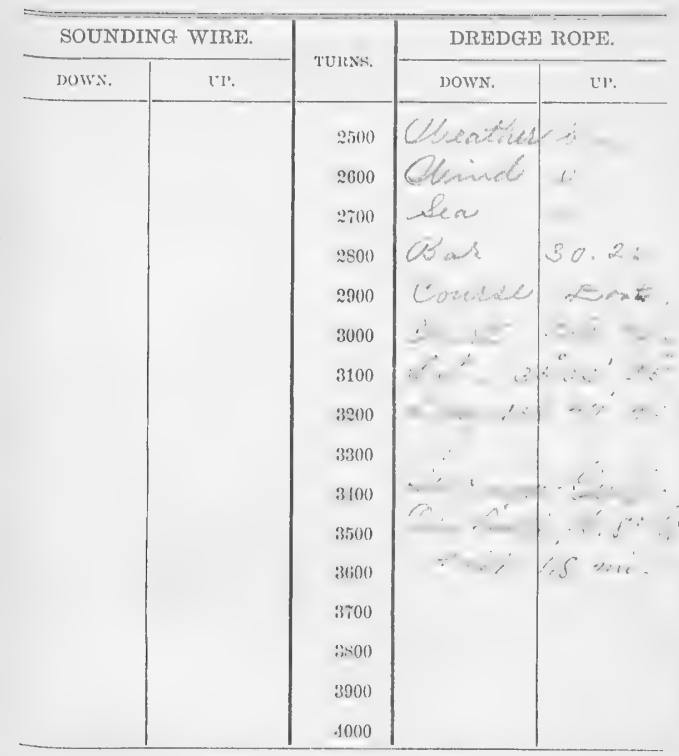

SERIAL TEMPERATURES,

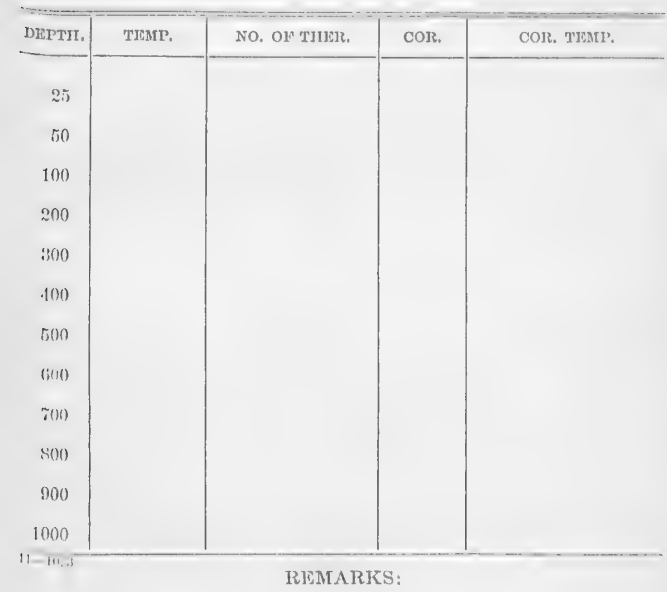

Sint 1 -eerat 
No. dil

Zercas

Date

\section{Lucas Machine. Recl. Seceat}

Turns

Cor: +

Depth $5>70+2$

Shot on toad

3s=

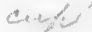

Bottom

(?)

Bottom temperature $71.0^{\circ}$

No. of themometer $/ 88^{4} / s^{-n} s^{-}$

Cor.

Corrected temperature

$$
70.8^{\circ}
$$

Air. 87

Surface ) 3*

Drift

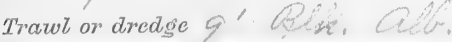

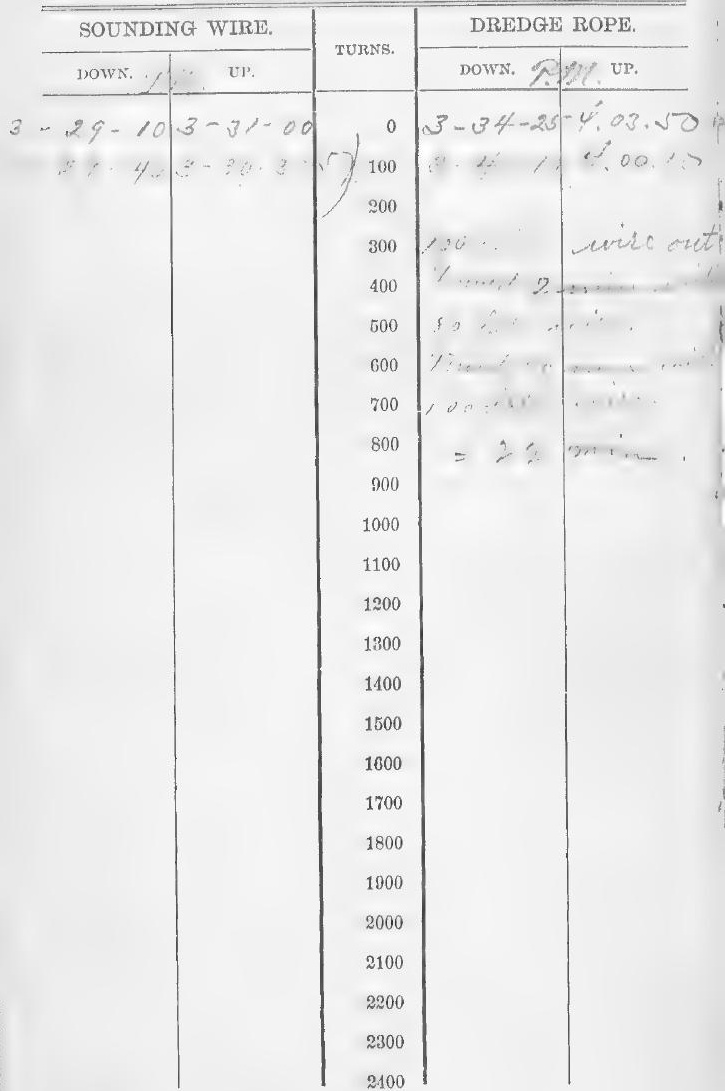




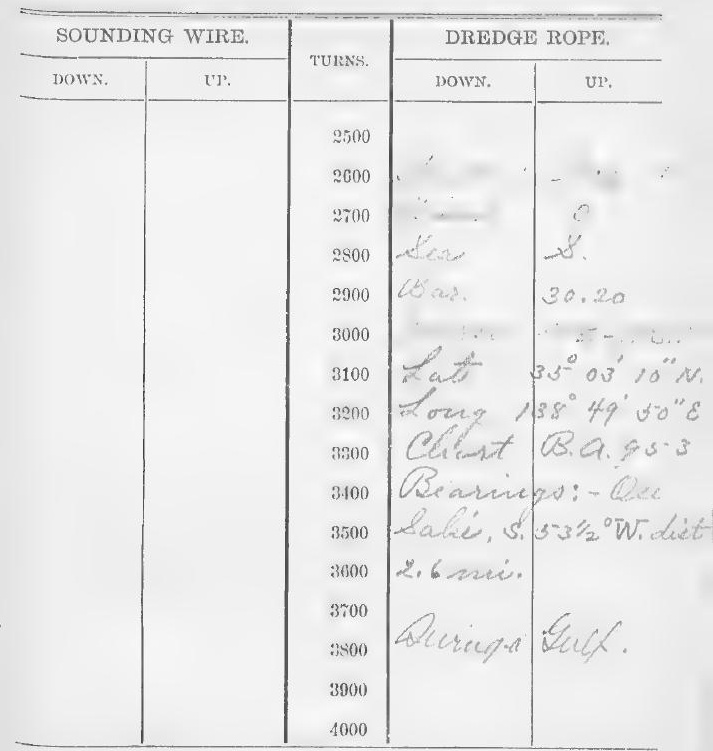

SERIAI TEMPERATURES.

\begin{tabular}{|c|c|c|c|c|}
\hline DEPTII. & 'ТЕМР. & NO. OF THELI. & corr. & COIR. THAII. \\
\hline 25 & & & & \\
\hline 50 & & & & \\
\hline 100 & & & & \\
\hline 200 & & & & \\
\hline$: 300$ & & & & \\
\hline 400 & & & & \\
\hline 500 & & & & \\
\hline bi10 & & & & \\
\hline 7100 & & & & \\
\hline 800 & & & & \\
\hline $5(11)$ & & & & \\
\hline 1000 & & & & \\
\hline
\end{tabular}


$\lambda \hat{s}^{\circ}$

No.

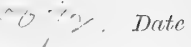

,

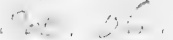

7 Less

Machine.

Recl.

\section{Turns}

Cor.t

Depth $28 \%$ from.

Shot ar-teat 35 - 4 ando SS Gyp

Bottom

$3 \%$. 32 .

Bottom temperature

No. of thermometer

$108 / 55$

Cor. - 2

\section{Corrected temperature}
Air $69^{\circ}$
Surface
Drift

Trawt or dredge of $\sqrt{1} / \mathrm{LG} . \ldots$.

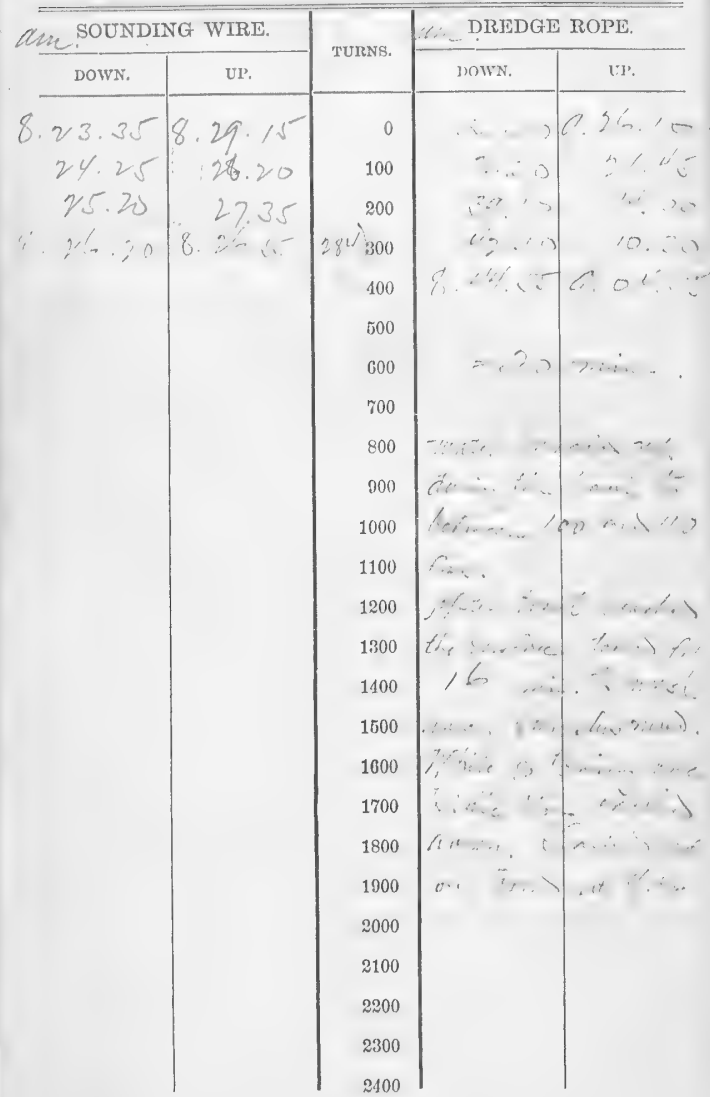




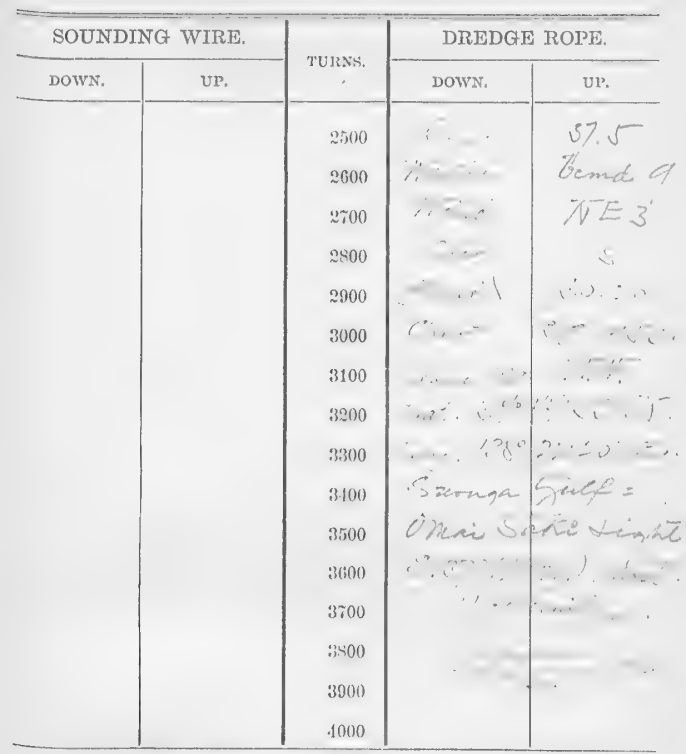

SERIAL TEMIPINARATURES.

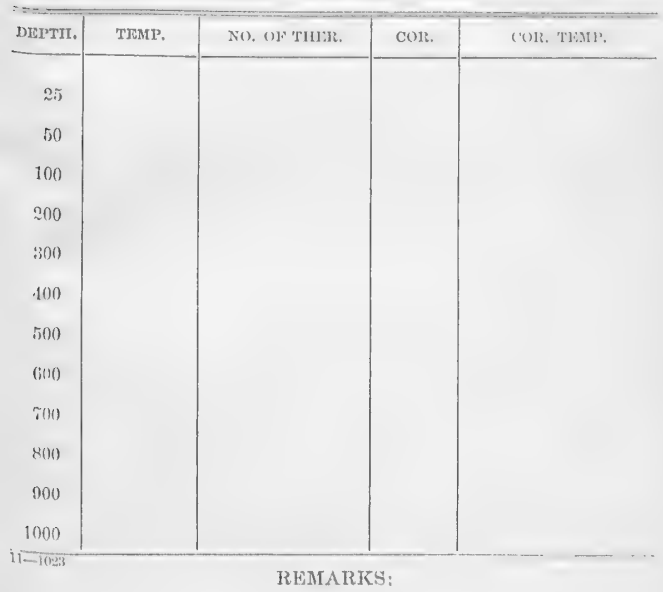


Dili No. 0.5073. Date 16 Oct. 1906. Lineno Machine. Reel. Zueno
Turns
Cor.+
Depth 148 fino.
Shot as Leard.
Bottom
9y

Bottom temperature

No. of thermometer $108 / \sqrt{5}$.

Corrected temperature

Cor. -.2

Air $72 \circ$ Surface 75:

Trawt or dredge $q$ ' $\$ 1 L 6 .-18$.

Drift

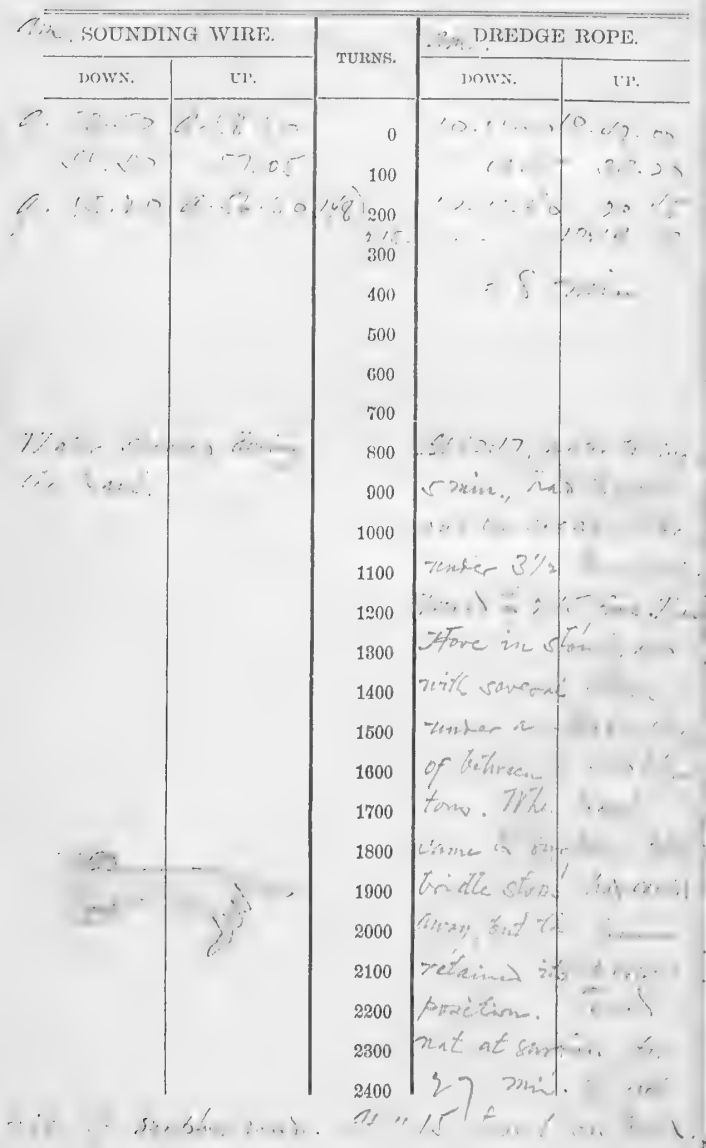




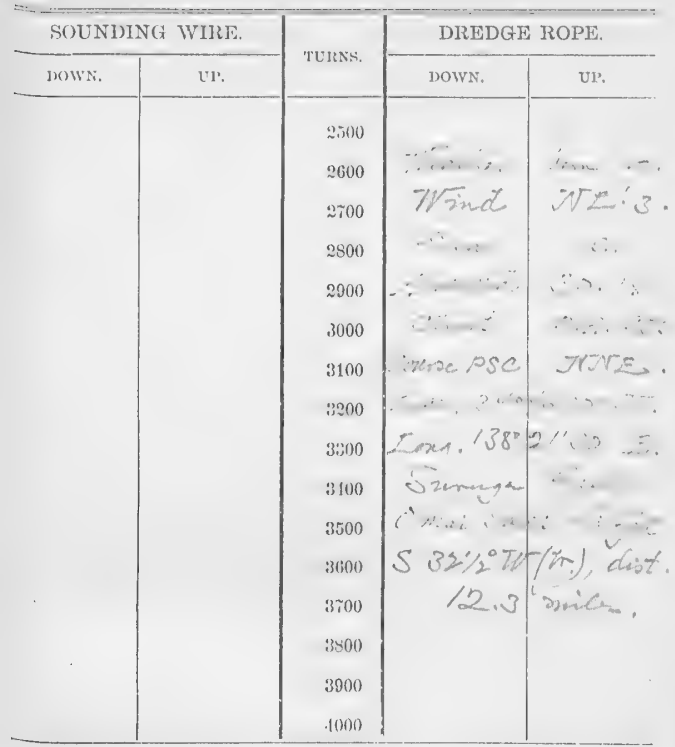

SEIRIAL TEMPERATURES

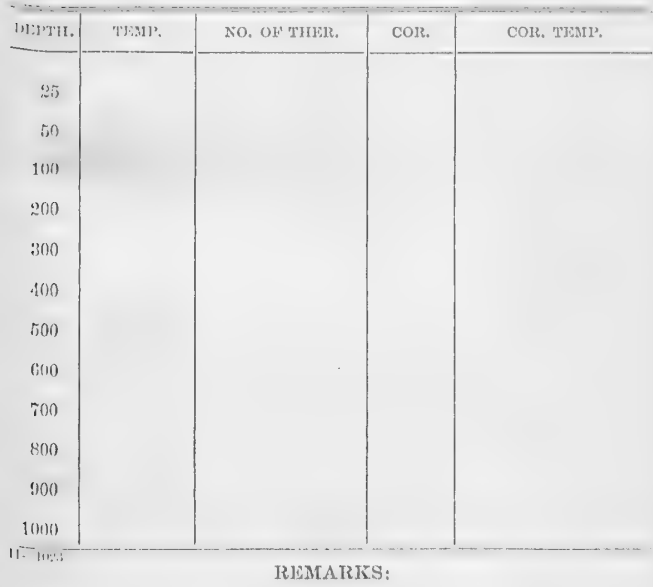


No.f \& 7 Date

Machine. Reel.

Turns Cor.t Depth \& .

Shot or lead 35 .

Bottom

Bottom temperature

No. of thermometer $1 . \quad$ Cor.-...

Corrected temperature

Air \ 5 Surface $y 3$ Drift

Trawt or aredge

\section{SOUNDING WIRE.}

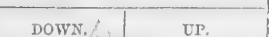

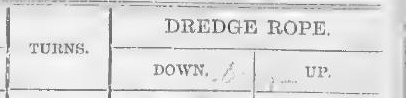




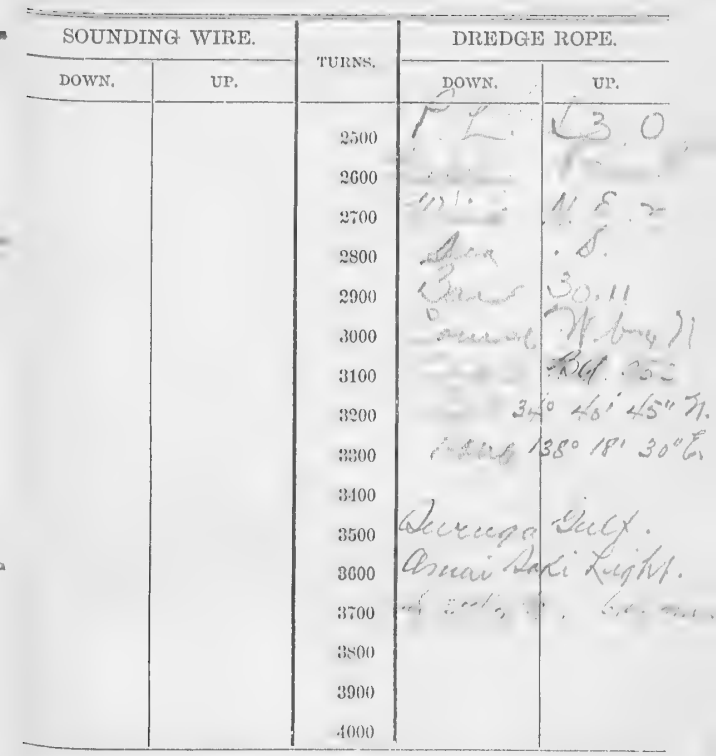

SERTAJ, TEMPERATURES

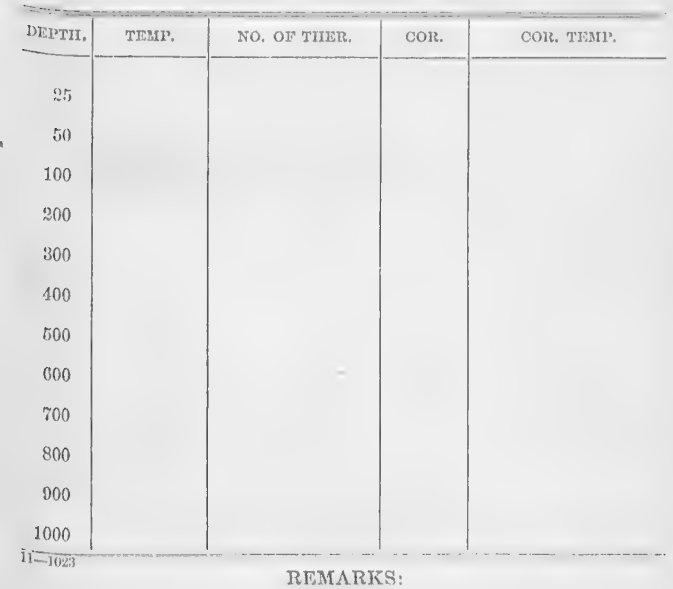




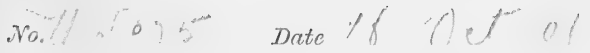

$$
\text { TH Mactuine. Reel. }
$$

Turns Cor.t Depth 22

Shot or lead 3

Bottom, ou $C_{2}, 5$

Bottom temperature $\{5,2$

No. of thermometer / $/ 15$ Cor. -2

\section{Corrected temperature 7$\}$}

Air. Surfure Jritl.

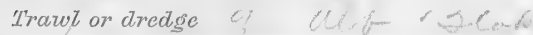

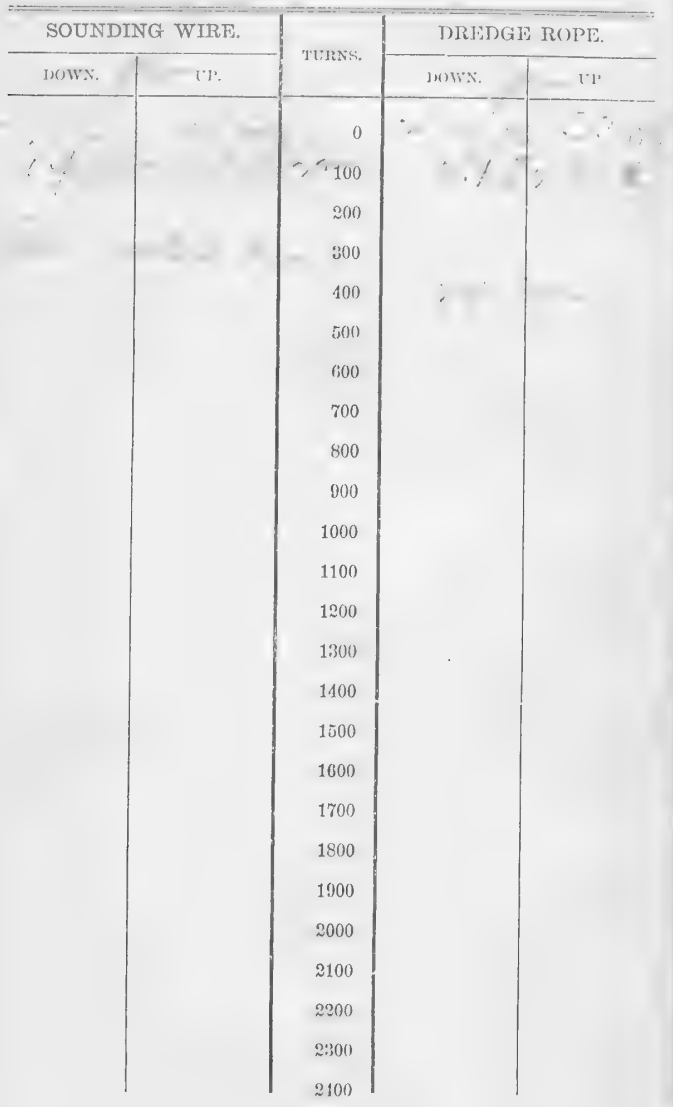




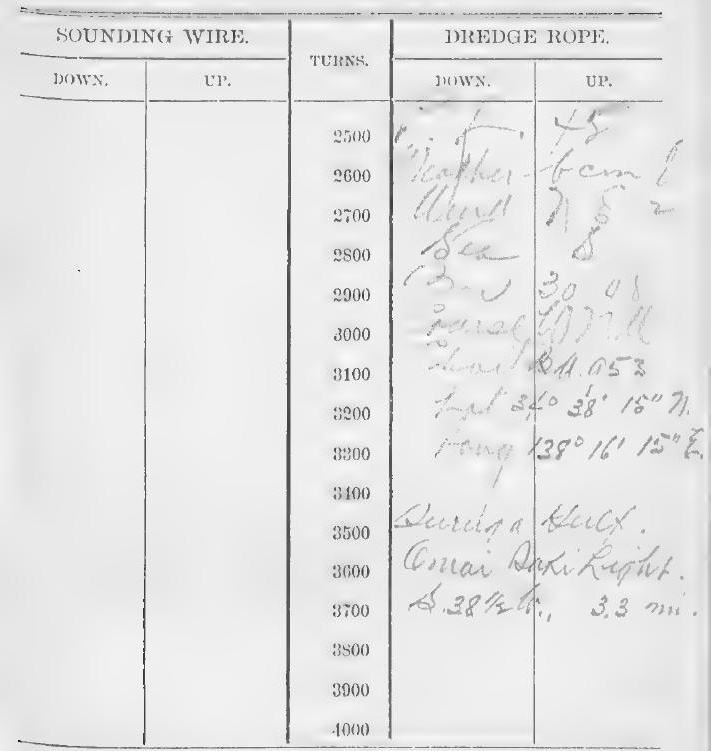

SERIAL TEMPERATURES

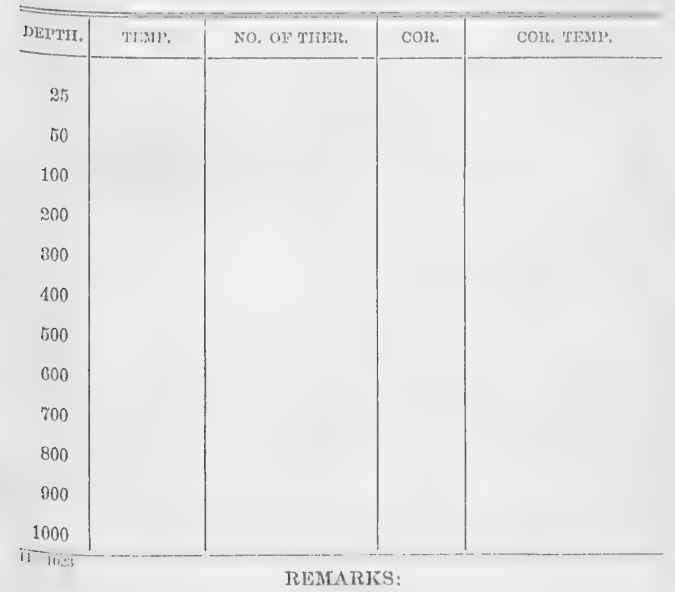


$\operatorname{tog} \hat{n}^{\mathrm{in} j}$

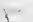

in. \& $\%$

J)rile

ucae

Mactine.

Rect. tuecent-

Turns

Cor + t

Depth 26 s- itath

Shot or teact $3 \mathrm{~s}^{- \text {th }}$ \& iquete cuge,

Bottom quif, his dif.

Bottom temperature $4.4 .0^{\circ}$

No. of thermometer $108 / \mathrm{SH}^{-}$

Cот $-12^{\circ}$

Corrected temperature $43.8^{\circ}$
Aiv.
Surfiere $7 \mathrm{i}$
7)rift

Trawt or dreage o' QPe teik

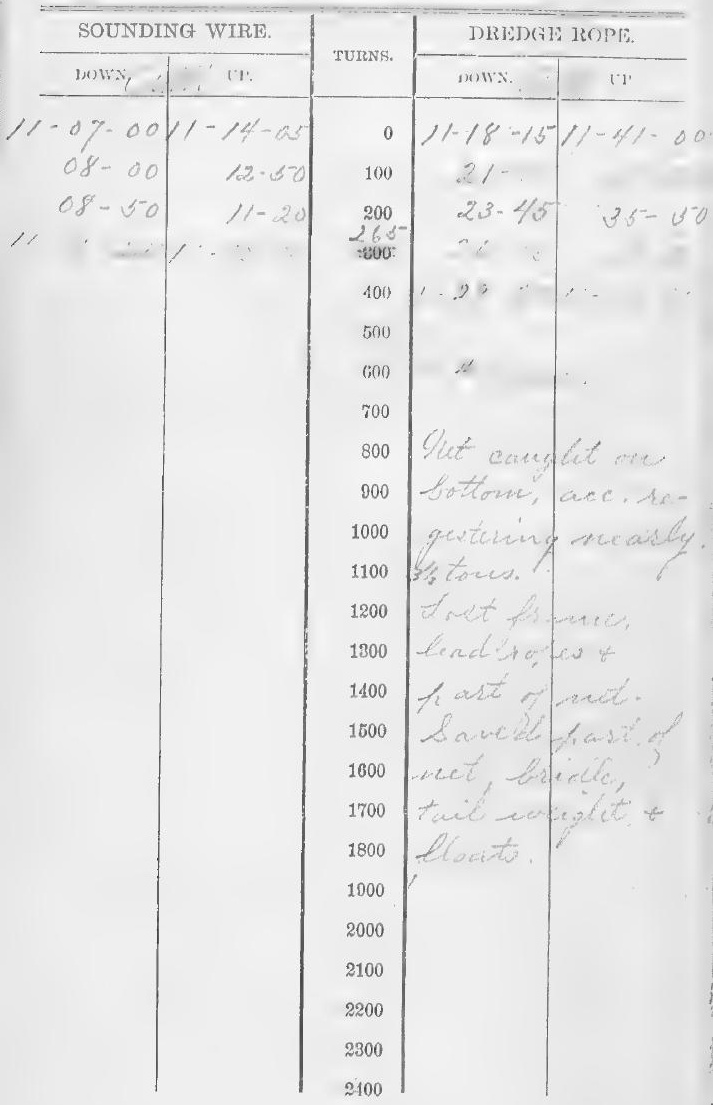




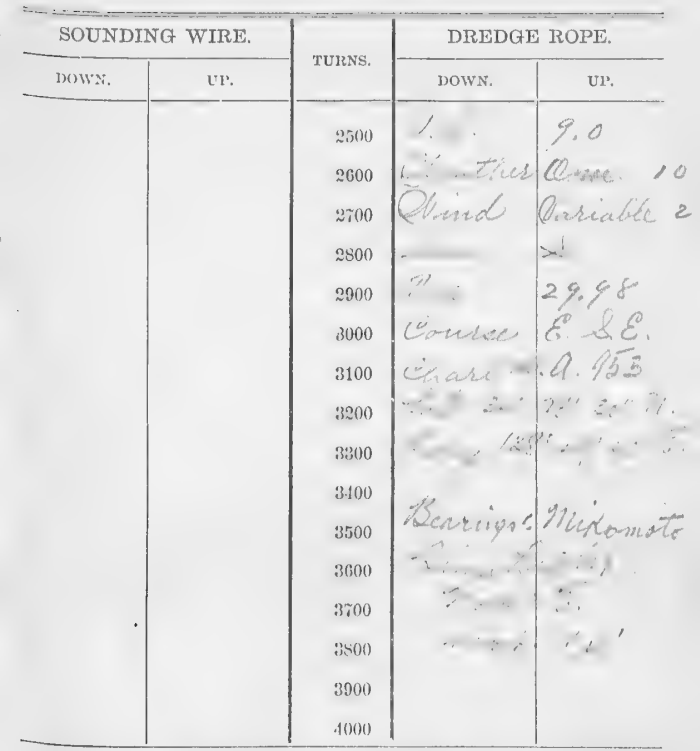

SEIRIAT, TEMPERATURES

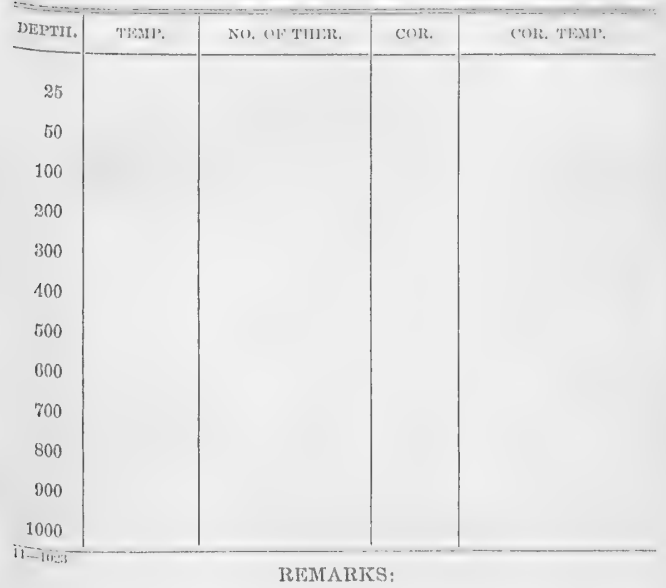


gi No. H. Y895. Date 170 ct. 1006 .

Luens Machine. Reel. Thuers

Turns Cor.t Depth $36 /$ fro.

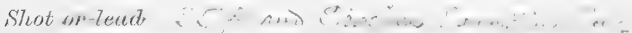

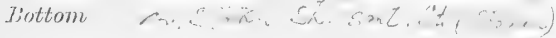

Bottom temperature $39: 8$

No. of thermometer $108 / \sqrt{6}$ Cor. - . 2

Corrected temperature $39: 6$

Air $750^{\circ}$ Surface 75 Drift

Trawt or dredge

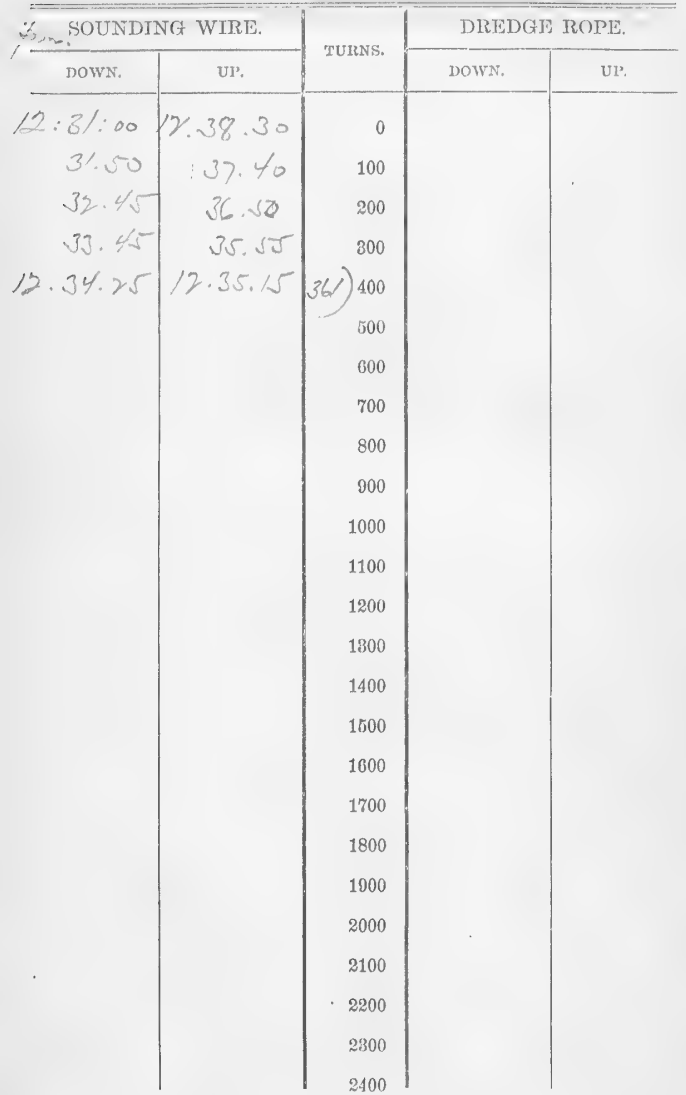




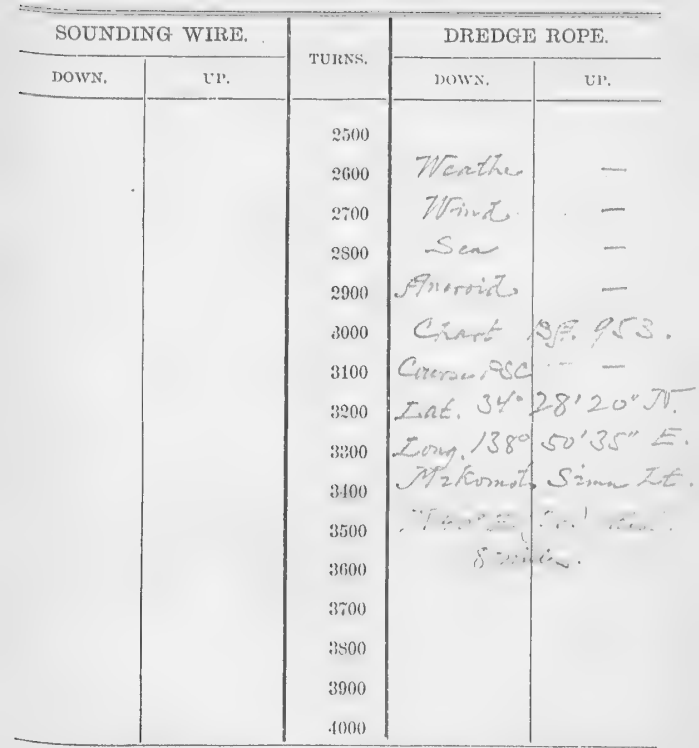

SERILL TEAIPERATURES

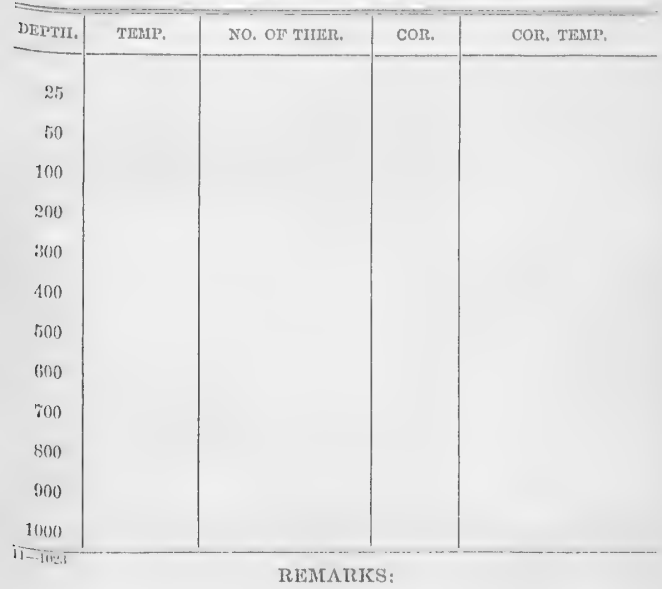


Reel, Tums Cor.t Depth 520 ro. Shotrazlead BV 4 ams S. S. Cins.

\section{Bottom}

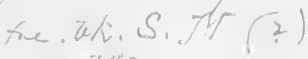

Bottom temperature

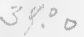

No. of thermometer 108155

Cor. -. I

Corrected temperature 38.9

Ais

Surfuce

- Drift

Trewe or alredige

$=\%$, SOUNDING WIRE.

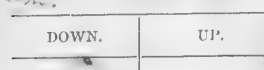

2. $11,5.5$

u :

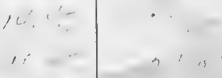

17.

$7, \ldots$

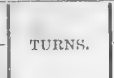

$2 \ldots$

$\therefore$ Trí, $\cdots$, $\therefore$

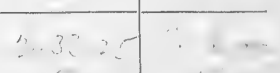


No. 10. voy8. Date. Qet. 19, 1906
tucae
Machine.
Reel.

Turns

Cor.t

Depth 1 it fath.

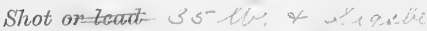

Bottom Rive. q.y, s, If l.t.

Bottom temperature $39 \%^{\circ}$

No. of thermometer $108 / S^{-6}$

Cor. - . ${ }^{\circ}$

Corrected temperature 38,9

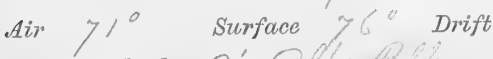

Trawt or aredge g' QPbi the

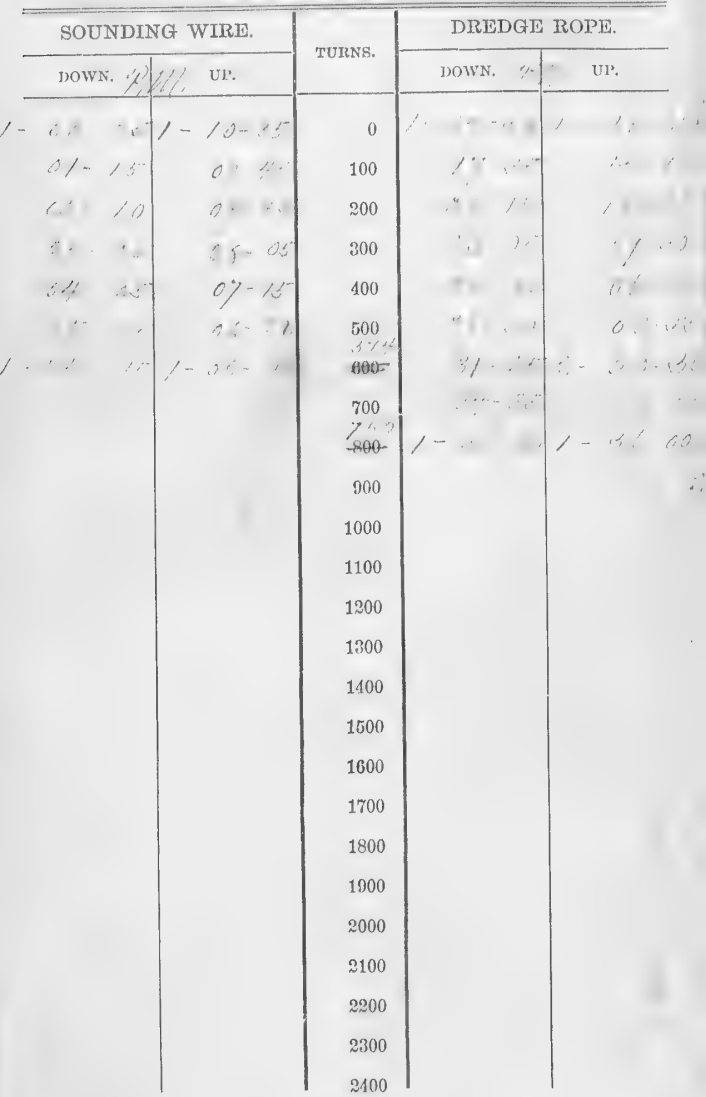




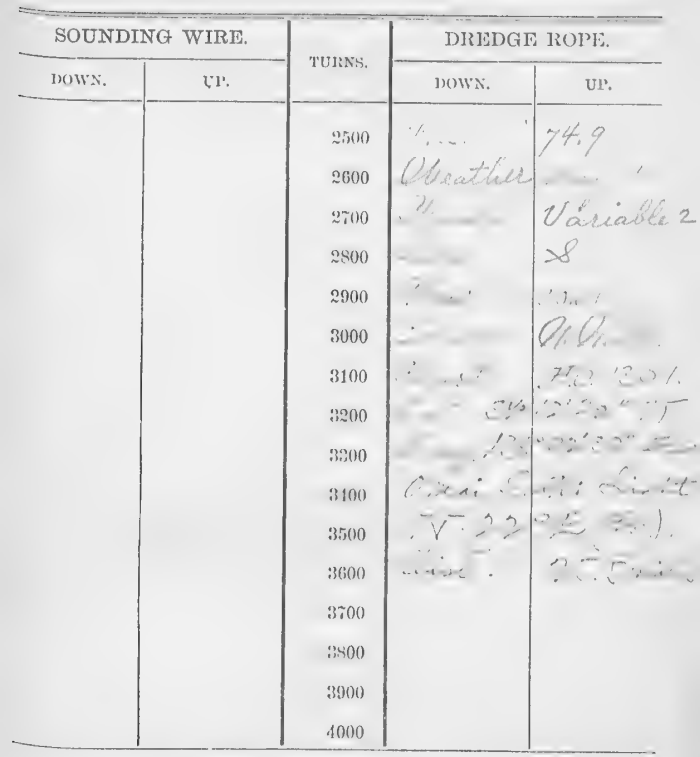

SERIAL TEMPERATURES.

\begin{tabular}{|c|c|c|c|c|}
\hline DЕРTI. & THMP. & NO. OF THET. & corr. & COL. TIENL. \\
\hline 25 & & & & \\
\hline 50 & & & & \\
\hline 100 & & & & \\
\hline 200 & & & & \\
\hline ;i)0 & & & & \\
\hline 101$)$ & & & & \\
\hline 500 & & & & \\
\hline$f(1)$ & & & & \\
\hline $5(10)$ & & & & \\
\hline 800 & & & & \\
\hline 900 & & & & \\
\hline 1000 & & & & \\
\hline
\end{tabular}

REMLARKS:

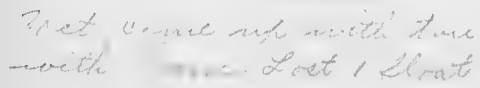


1.

No. 60

Rucase

S.0.9.

Date Q et.

Machine.

Reel.

Turns

$\mathrm{Cor} \cdot+$

Dopt

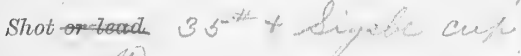

Bottom $\%$.

Bottom, temperature

No, of thermometer $108 / 5^{-} \quad$ Cor. $-.1^{\circ}$

Corrected temperature 39, ${ }^{\circ}$

Air $\because s^{\circ} \quad$ Surfuce $\because$ Drift

Trawl or dredge $q^{\prime}$ Ceraesers

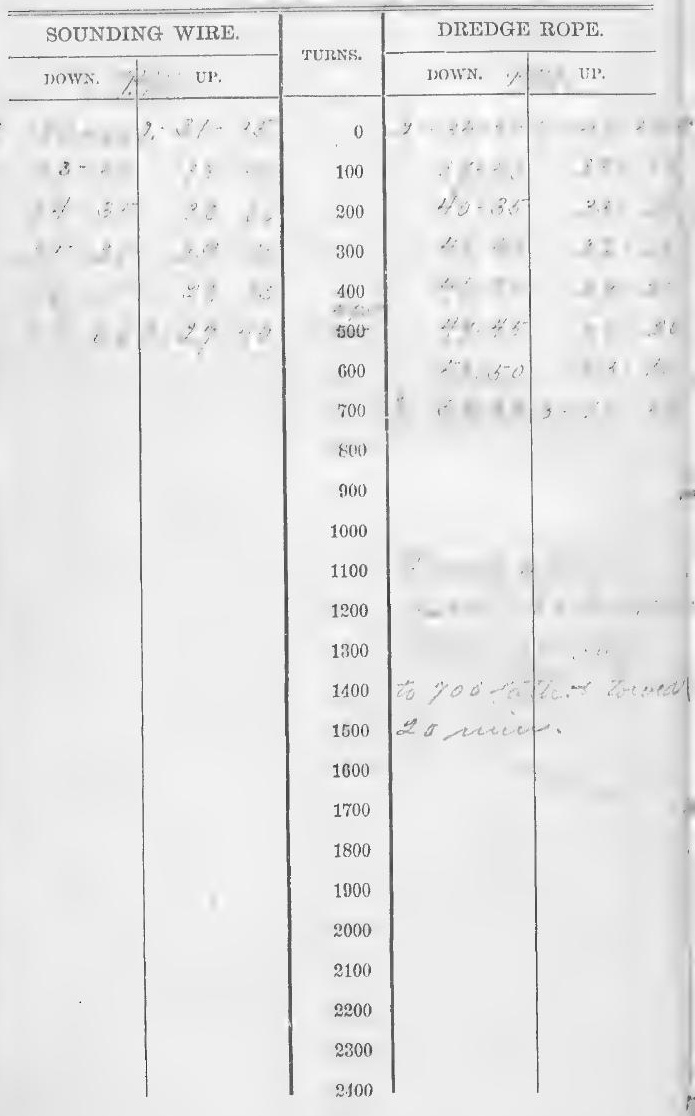


No.

Date

ita Machine.

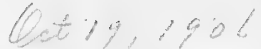

\section{Turns}

Cor. +

Reel.

Shot or teate $33^{\text {th }}$ of lig abe cuph

Bottom fre. $7 \%$. S. C. 406.

Bottom temperature $38.8^{\circ}$

No. of thermometer $/ 08 / \mathrm{S}^{-}$Cor.,$- 1^{2}$

Corrected temperature $38.7^{\circ}$

Air $72^{\circ}$ Surface $75^{\circ}$ Drift

Trawt or dredge $9^{\prime} \mathrm{A} g \mathrm{arbi}^{\prime}$

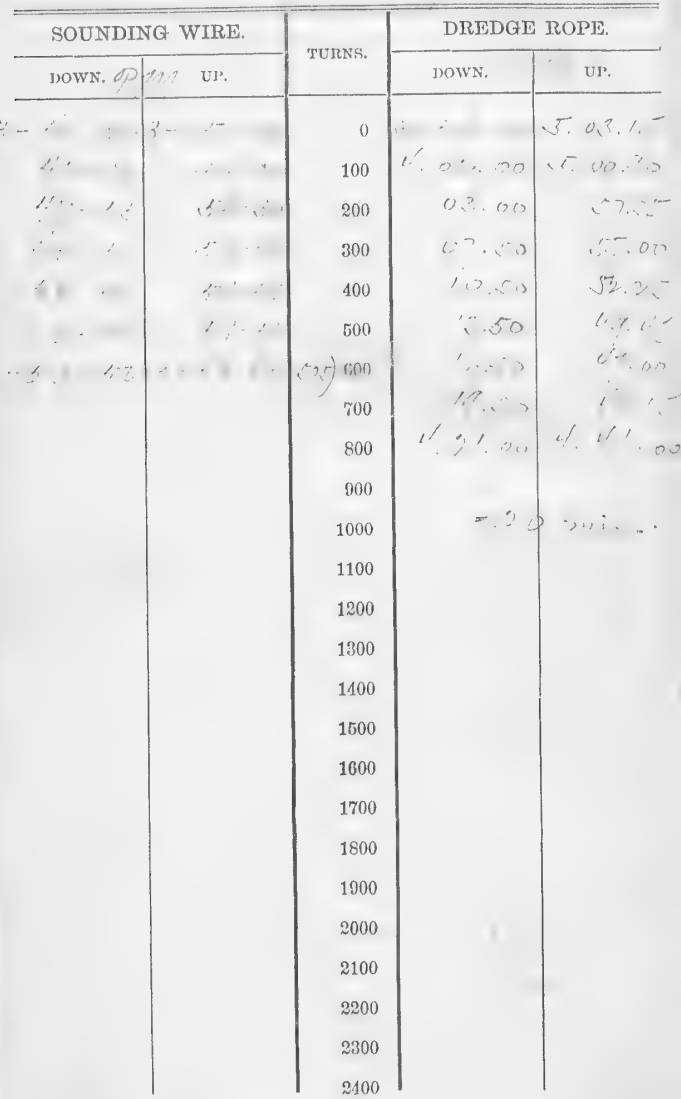




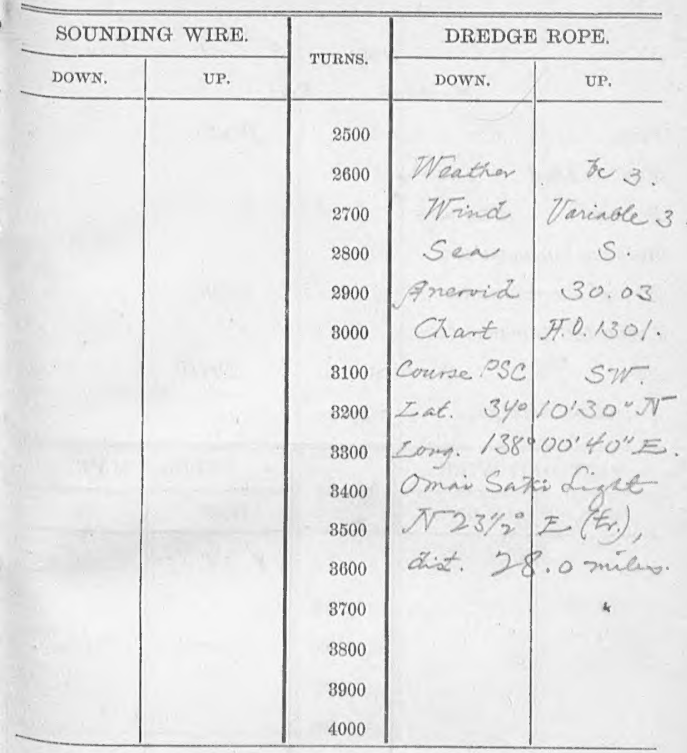

SERIAL TEMPERATURES.

\begin{tabular}{|c|c|c|c|c|}
\hline DEPTH. & TEMP. & NO. OF THER. & COR. & COR. TEMP. \\
\hline 25 & & & & s. \\
\hline 50 & & 1) & & \\
\hline 100 & & & & \\
\hline 200 & & & & \\
\hline 800 & & & & \\
\hline 400 & & & & \\
\hline 500 & & & & \\
\hline 600 & & & & \\
\hline 700 & & & & \\
\hline 800 & & & & \\
\hline 900 & & & & 4 \\
\hline 1000 & & & & \\
\hline
\end{tabular}

REMARKS: 
Machine, Reel.

Turns

Cor.t

Depth

Shot or lead

\section{Bottom}
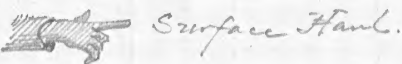

\section{Bottom temperature}

No. of thermometer

Cor.

\section{Corrected temperature}

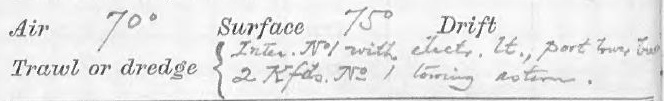

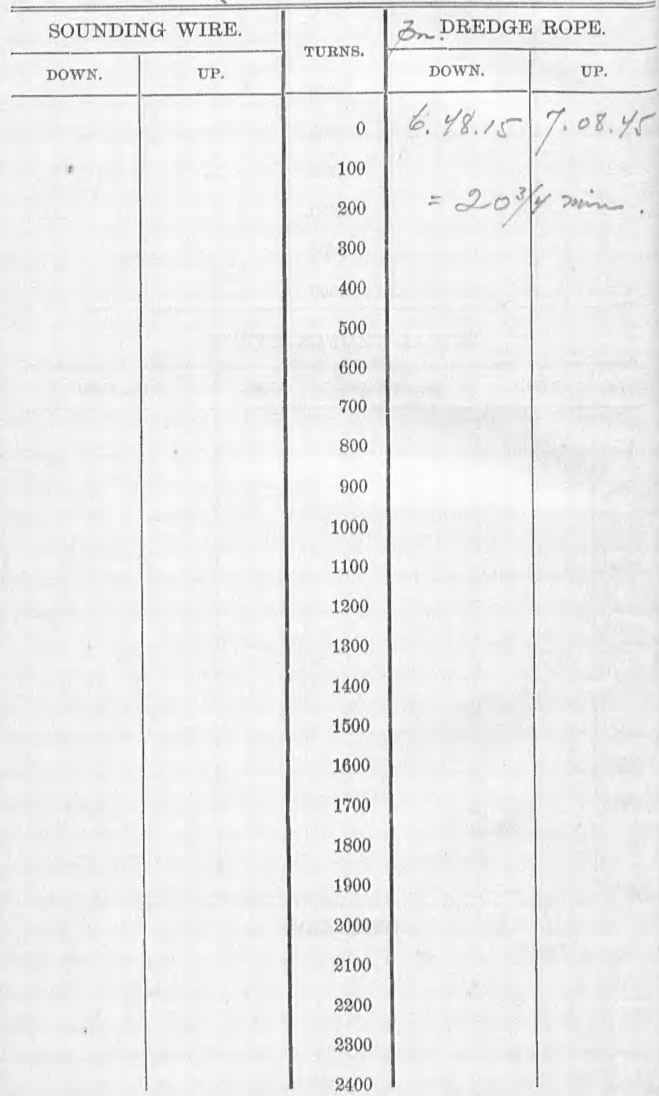




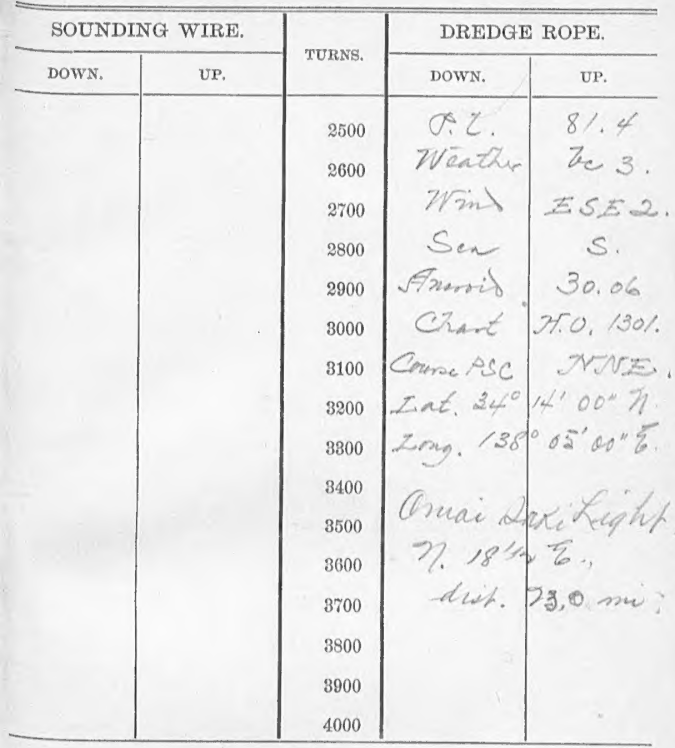

SERIAL TEMPERATURES,

\begin{tabular}{|c|c|c|c|c|}
\hline DEPTH. & TEMP. & NO, OF THER. & COR. & COR. TEMP. \\
\hline 25 & & & & \\
\hline 50 & & & & \\
\hline 100 & & & & \\
\hline 200 & & & & \\
\hline 300 & & & & \\
\hline 400 & & & & \\
\hline 500 & & & & \\
\hline 600 & & - & & \\
\hline 700 & & & & \\
\hline 800 & & & & \\
\hline 900 & & & & \\
\hline 1000 & & & & \\
\hline
\end{tabular}

REMARKS: 
we 3.28

$108021-10$
$104914-15$
$108154-25$

$108019-100$

$\operatorname{Tin} 8 \overline{0.2-100}$

$108155908244-100$

$$
\frac{1-643}{52}=
$$

$L_{D}=$ ove $x^{\prime}-\bar{t}$

$$
\begin{aligned}
& \operatorname{lin} x \text { mento } \\
& \text { a } b \\
& \text { nimbor }
\end{aligned}
$$

$164 \pi / \cos ^{2} \sin \cdot \operatorname{lin} 2$ (5)

$\log 8$

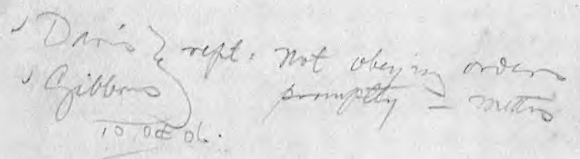

